Check for updates

Cite this: J. Mater. Chem. B, 2020, 8, 3261

Received 7th October 2019, Accepted 13th November 2019

DOI: $10.1039 / c 9 t b 02189 b$

rsc.li/materials-b

\title{
Biosensors and bioassays for determination of matrix metalloproteinases: state of the art and recent advances
}

\author{
Zhen Lei, ${ }^{\text {ab }}$ Minghong Jian, ${ }^{\mathrm{b}}$ Xiaotong Li, ${ }^{\mathrm{b}}$ Jia Wei, ${ }^{\mathrm{c}}$ Xianying Meng ${ }^{{ }^{\mathrm{c}}}$ and \\ Zhenxin Wang (iD $* b$
}

\begin{abstract}
Matrix metalloproteinases (MMPs) are closely associated with various physiological and pathological processes, and have been regarded as potential biomarkers for severe diseases including cancer. Accurate determination of MMPs would advance our understanding of their roles in disease progression, and is of great significance for disease diagnosis, treatment and prognosis. In this review, we present a comprehensive overview of the developed bioassays/biosensors for detection of MMPs, and highlight the recent advancement in nanomaterial-based immunoassays for MMP abundance measurements and nanomaterial-based biosensors for MMP activity determination. Enzyme-linked immunosorbent assay (ELISA)-based immunoassays provide information about total levels of MMPs with high specificity and sensitivity, while target-based biosensors measure the amounts of active MMPs, and allow imaging of MMP activities in vivo. For multiplex and high-throughput analysis of MMPs, microfluidics and microarray-based assays are described. Additionally, we put forward the existing challenges and future prospects from our perspective.
\end{abstract}

\section{Introduction}

Matrix metalloproteinases (MMPs) are a family of extracellular $\mathrm{Zn}^{2+}$-dependent endopeptidases; they can degrade extracellular matrix (ECM) proteins and the main components of the basement membrane, including collagen, gelatin, fibronectin and proteoglycan. ${ }^{1}$ Since the first discovery in 1962, nearly 30 human MMPs have been identified, and are labeled as MMP-1 to MMP-28. ${ }^{2}$ According to their substrate specificity and structural similarity, MMPs are normally classified into six subgroups, i.e., collagenases, gelatinases, stromelysins, matrilysins, membrane-type MMPs (MT-MMPs) and others. MMPs display high sequence homology, and they are generally comprised of several domain motifs, including a signal peptide at the $\mathrm{N}$-terminal for guiding the translated protein into the endoplasmic reticulum, a propeptide domain with a conserved "cysteine switch" to maintain the latency of pro-MMPs, a catalytic domain containing a $\mathrm{Zn}^{2+}$ binding motif for MMP

\footnotetext{
${ }^{a}$ School of Chemistry and Environmental Engineering, Wuhan Institute of Technology, Wuhan, 430205, P. R. China

${ }^{b}$ State Key Laboratory of Electroanalytical Chemistry, Changchun Institute of Applied Chemistry, Chinese Academy of Sciences, Changchun, Jilin 130022, P. R. China.E-mail: wangzx@ciac.ac.cn

${ }^{c}$ Department of Thyroid Surgery, The First Hospital of Jilin University, Changchun, Jilin 130021, P. R. China. E-mail: xy6823@163.com
}

proteolytic activity, a hinge region for connecting the catalytic domain and the hemopexin-like domain, and a C-terminal hemopexin-like domain for regulating the interaction of MMPs with substrates, tissue inhibitors of metalloproteinases (TIMPs) and the cell surface. ${ }^{3}$ Some MMPs contain other additional domains for particular functions, for example, MT-MMPs have cytoplasmic and transmembrane domains for anchoring them to the cell surface.

MMPs are secreted as latent zymogens, and need activation to display their proteolytic activity. The expression levels and activities of MMPs are controlled at three levels, namely, transcription of MMP genes, activation of zymogens and inhibition of active MMPs. ${ }^{4}$ MMPs are mainly regulated by the mitogenactivated protein kinase (MAPK) signal transduction pathway, thus the transcription of MMPs may be induced by various factors, such as cytokines, growth factors, chemokines and chemical agents. ${ }^{5}$ When secreted as a pro-MMP, the cysteine residue $\left(\mathrm{Cys}^{73}\right)$ in the propeptide domain interacts with $\mathrm{Zn}^{2+}$ in the catalytic domain, forming a bond and keeping the MMP inactive. The activation of MMPs is realized by disrupting the bond between the cysteine residue and $\mathrm{Zn}^{2+}$, which is known as the cysteine switch. MMPs can be activated not only by kinds of intracellular proteases, including plasmin, trypsin, and certain MMPs (MMP-3, MMP-14), but also by exogenous chemical agents, such as 4-aminophenylmercuric acetate (APMA), denaturants and reactive oxygen. To maintain normal physiological functions, the activities of 
MMPs are regulated by endogenous inhibitors (e.g., TIMPs, $\alpha 2$-macroglobulin) in tissue and blood circulation to keep a good balance. ${ }^{3,4}$ MMPs are secreted by a variety of connective tissues and proinflammatory cells, and participate in normal physiological processes, such as embryonic development, organ morphogenesis, bone remodeling, nerve growth and wound healing. However, dysregulation of expression and activities may result in severe diseases, such as arthritis, cardiovascular disease, neurodegenerative disease and cancer. ${ }^{6}$ It has been reported that overexpression of MMP-1, MMP-8, and MMP-13 is observed in rheumatoid and osteoarthritis, resulting in erosion of cartilage. ${ }^{7,8}$ MMP-1 and MMP-9 are found to be involved in atherosclerotic lesion formation. ${ }^{9}$ Increased expression levels and activities of MMPs have been proved in almost all types of human cancers. Due to the involvement in several aspects of cancer development, for instance, growth and apoptosis of cancer cells, tumor-induced angiogenesis, tumor invasion and metastasis and immune responses to cancer, MMPs have been regarded as the hallmarks of cancers. ${ }^{3}$

Current knowledge demonstrates the participation of MMPs in physiological and pathological processes, particularly in tumor development and progression. Since the functions of MMPs in tumors are not yet fully elucidated, unraveling the roles of specific MMPs in disease progression is an enormous challenge. Accumulated evidence indicates that certain MMPs can serve as potential biomarkers for disease diagnosis and prognosis, therefore detecting MMPs in vitro and in vivo with high accuracy and sensitivity is of great significance. Up to now, massive efforts have been devoted to developing assays/ biosensors for determining MMPs' abundance and their activities. There are already some reviews summarizing the related assays, ${ }^{10-16}$ but they mostly concentrated on the commonly used immunoassays, zymography and fluorescence assays. Besides a brief overview of some current measurement approaches, the latest published review on MMP detection mainly focuses on a summary of biosensors based on detection principles such as electrochemical and surface plasmon resonance (SPR)-based and other optical biosensors. ${ }^{17}$ The last decades have witnessed rapid progress in methodology and nanotechnology; nanomaterials with unique properties have shown great promise for developing biosensors. With the growing interest in MMPs, numerous novel bioassays and biosensors combining particular nanomaterials with different detection principles have been proposed for sensitive MMP detection in vitro and in vivo.

Herein, we present a comprehensive summary about existing bioassays and biosensors for determination of MMP concentrations and activities (Fig. 1), and highlight recent advances in achieving high sensitivity, real-time and in vivo detecting ability and multiplexing capability with the aid of the unique properties of nanomaterials. Firstly, we describe the recent progress of immunoassays/immunosensors for quantifying MMP concentrations. Subsequently, updated target-based biosensors for measuring MMP activities are addressed, followed by the burgeoning microfluidics-based platform for multiplex analysis. Additionally, some less commonly used methods are also

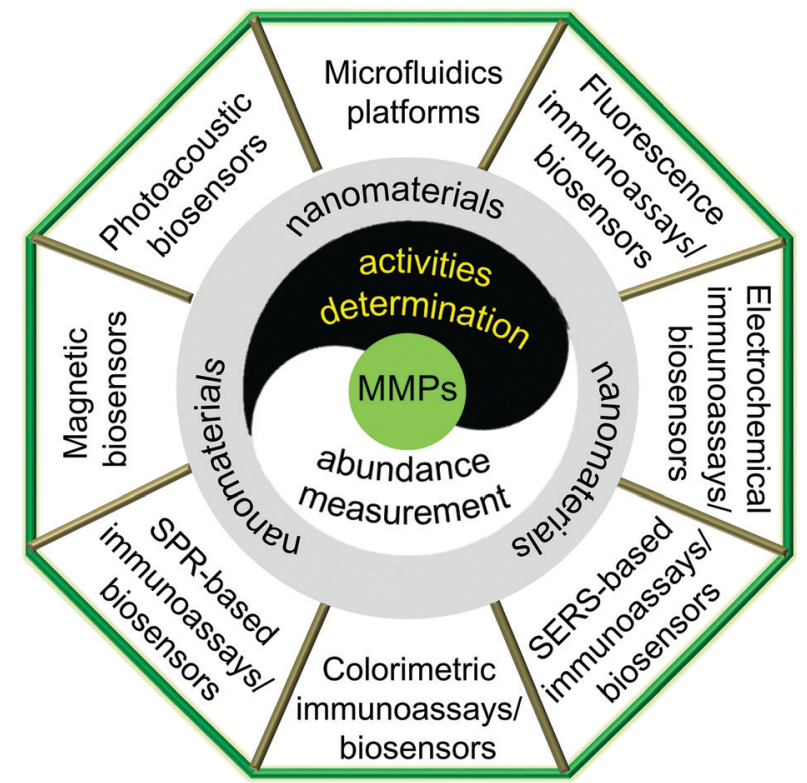

Fig. 1 Summary of current immunoassays and biosensors for determination of MMP abundance and activities.

discussed, such as liquid chromatography tandem mass spectrometry (LC-MS/MS) and microarrays. Finally, we discuss the current challenges and put forward future prospects from our perspective. It is anticipated that this review will inspire researchers to develop more innovative approaches for facile and sensitive detection of MMPs in clinical samples, especially for point-of-care (POC) testing.

\section{Immunoassays/immunosensors}

Since the early 1990s, a variety of immunological methods based on the specific recognition between antigens and antibodies have been proposed to determine the amount of MMPs, such as Western blotting, immunohistochemistry, enzyme-linked immunosorbent assay (ELISA) and immunocapture assay.

\subsection{Western blotting}

Western blot analysis combines electrophoretic separation with immunostaining. After samples are separated by polyacrylamide gel electrophoresis (PAGE), MMPs are transferred as blots from the gel to a solid support, such as nitrocellulose membranes. Subsequently, the MMP proteins in the blots are recognized by specific primary antibodies, and then bind with horseradish peroxidase (HRP) conjugated secondary antibodies for staining. The colors of specific bands are generally developed by HRP-catalyzed chemiluminescence (CL) with luminal or isoluminal as substrates. ${ }^{18,19}$ In the presence of an enhancer, the CL intensity can be increased over 1000 fold. Information about the expression of specific MMPs can be obtained by analysing the location and intensity of the staining bands. However, this method is quite complicated and timeconsuming. 


\subsection{Immunohistochemistry assay}

Similar to Western blotting, immunohistochemistry (IHC) provides information about the localization and amount of target proteins in tissues or cells in situ. MMPs in tissue sections or cell specimens are captured by specific primary antibodies, and then detected by secondary antibodies conjugated with fluorescent molecules, ${ }^{20} \mathrm{HRP}^{21}$ or alkaline phosphatase (ALP). ${ }^{22}$ The fluorescence stained samples can be directly observed with a fluorescence microscope, which is also known as immunofluorescence staining (IF). IF is sensitive, simple and fast, but it may suffer from photobleaching. In the enzymebased chromogenic assays, samples are further stained with specific staining kits and counterstained with hematoxylin for optical microscope examination, which shows distinct advantages of longer storage time and better contrast. Particularly, the alkaline phosphatase/anti-alkaline phosphatase (APAAP) method can reduce the influence of endogenous enzymes, improving the sensitivity. With the development of advanced imaging techniques, gold nanoparticles (AuNPs) were conjugated on secondary antibodies, and served as labels in IHC, which was referred to as the immune colloidal gold technique. Mazzoni et al. first applied AuNP-based IHC analysis to evaluate the localization and distribution of MMP-2 and MMP-9 in a human dentin organic matrix by correlative field emission in-lens-scanning electron microscopy (FEI-SEM) and transmission electron microscopy (TEM).$^{23}$ Because AuNP labels can be easily identified and counted by electron microscopy (EM), the AuNP-based IHC analysis exhibits ultrasensitivity, with the eventual goal of detecting single MMP recognition events.

\subsection{ELISA}

ELISA is the gold standard for quantitative analysis of proteins, and has been widely used in clinical detection. The biorecognition events between target proteins and capture antibodies in conventional ELISA are usually characterized by colorimetric or fluorescent readout. The most frequently used colorimetric ELISA measures the absorbance of the colored product resulting from the enzyme-catalyzed chromogenic reaction of exogenous substrates. Although the assay allows determination of MMPs with the naked eye, it is still challenging to analyse low-abundance MMPs in physiological samples. Recently, with the emergence of various new nanomaterials, many novel ELISA-based approaches integrated with innovative detection techniques and signal amplification strategies have been developed to improve the assay performance, such as enhancing the sensitivity and shortening the assay time (Table 1).

2.3.1 Colorimetric/fluorescence immunoassay. Traditional ELISA is carried out in well plates, the repeated washing makes it rather time-consuming, and the sensitivity needs to be further improved. The employment of magnetic beads (MB) provides a versatile strategy for developing enhanced immunoassays. Compared with two-dimensional bulk solid surfaces, MBs with higher specific surface area can provide many more active sites for conjugation with antibodies, and promote the immunorecognition with target proteins, leading to faster reaction kinetics. Moreover, due to the magnetic property of MBs, they are easier to separate from other sample components by an external magnetic field, shortening the assay time. For instance, Ismail et al. developed a rapid and simple magnetoELISA for detecting MMP-9 by employing MBs to load monoclonal capture antibodies (c-MAb). ${ }^{24}$ The conventional multi-step ELISA was simplified into two steps by incubating c-MAb conjugated MBs with the analyte and biotinylated anti-MMP-9 polyclonal detection antibody (bd-PAb) simultaneously, and then labeling with poly-HRP (HRP polymers) for color development and signal amplification. Owing to the combination of MBs and poly-HRP, the classical ELISA carried out in $5 \mathrm{~h}$ could be shortened to $35 \mathrm{~min}$ with a detection limit of $11-30 \mathrm{pg} \mathrm{\textrm {mL } ^ { - 1 }}$. Similarly, Liang et al. proposed an immunomagnetic microparticle (IMP)-based time-resolved fluoroimmunoassay (TRF) to determine urinary MMP-7 in acute kidney injury (AKI) patients. $^{25}$ In the IMP-based TRF, one kind of anti-MMP-7 monoclonal antibodies were coupled on IMPs for capture, while another kind of MMP-7 antibodies were labeled with $\mathrm{Eu}^{3+}$ chelate for fluorescence detection. MMP-7 was detected via the one-step double-antibody sandwich immunoassay, which could be accomplished in $30 \mathrm{~min}$. Unlike the sandwich

Table 1 Assay performance of different immunoassays for measuring MMP concentrations

\begin{tabular}{|c|c|c|c|c|c|}
\hline Target & Sensing platform & Transduction type & Linear range & Detection limit & Ref. \\
\hline MMP-9 & MB/poly-HRP & Colorimetry & $16-1000 \mathrm{pg} \mathrm{mL}{ }^{-1}$ & $11 \mathrm{pg} \mathrm{mL}-1$ & 24 \\
\hline MMP-7 & IMP & Fluorescence & $0.063-150 \mathrm{ng} \mathrm{mL}^{-1}$ & $0.039 \mathrm{ng} \mathrm{mL}^{-1}$ & 25 \\
\hline MMP-2 & MSN & Fluorescence & $0.25-50 \mu \mathrm{g} \mathrm{mL}^{-1}$ & $0.02 \mu \mathrm{g} \mathrm{mL}^{-1}$ & 26 \\
\hline MMP-9 & MB/poly-HRP & Electrochemistry & $30-1000 \mathrm{pg} \mathrm{mL}^{-1}$ & $18-28 \mathrm{pg} \mathrm{mL}^{-1}$ & 28 \\
\hline MMP-9 & $\mathrm{MB} /$ poly-HRP & Electrochemistry & $0.03-2 \mathrm{ng} \mathrm{mL}^{-1}$ & $13 \mathrm{pg} \mathrm{mL}^{-1}$ & 29 \\
\hline MMP-2 & $\mathrm{Au}-\mathrm{NG} / \mathrm{PDA}-\mathrm{GO}$ & Electrochemistry & $0.0005-50 \mathrm{ng} \mathrm{mL}{ }^{-1}$ & $0.11 \mathrm{pg} \mathrm{mL}{ }^{-1}$ & 30 \\
\hline MMP-3 & SWCNT/polybead & Electrochemistry & $4-300 \mathrm{pg} \mathrm{mL} \mathrm{L}^{-1}$ & $4 \mathrm{pg} \mathrm{mL}-1$ & 31 \\
\hline MMP-2 & K-GS/aptamer & Electrochemistry & $10^{-4}-10 \mathrm{ng} \mathrm{mL}^{-1}$ & $35 \mathrm{fg} \mathrm{mL}^{-1}$ & 32 \\
\hline MMP-9 & PS@PDA/CdTe-QDs & Electrochemistry & $0.3-10000 \mathrm{pg} \mathrm{mL}^{-1}$ & $0.033 \mathrm{pg} \mathrm{mL}-1$ & 33 \\
\hline MMP-2 & PS@PDA-Ag NPs & Electrochemistry & $10^{-5}-10^{3} \mathrm{ng} \mathrm{mL}^{-1}$ & $5 \mathrm{fg} \mathrm{mL}-1$ & 34 \\
\hline MMP-2 & $\mathrm{TiO}_{2}$-NTs/CdS:Mn/CdTe & Photoelectrocemistry & $10 \mathrm{fg} \mathrm{mL}^{-1}-500 \mathrm{pg} \mathrm{mL}^{-1}$ & $3.6 \mathrm{fg} \mathrm{mL}^{-1}$ & 35 \\
\hline MMP-9 & CMD 50 D chip & SPR & $10-200 \mathrm{ng} \mathrm{mL}^{-1}$ & $8 \mathrm{pg} \mathrm{mL}^{-1}$ & 38 \\
\hline MMP-1 & Gold chips & SPRI & $0.05-20.00 \mathrm{ng} \mathrm{mL}^{-1}$ & $9 \mathrm{pg} \mathrm{\textrm {mL } ^ { - 1 }}$ & 39 \\
\hline MMP-3 & AuNPs & FOPPR & $0.05-50 \mathrm{ng} \mathrm{mL}{ }^{-1}$ & $34 \mathrm{pg} \mathrm{mL}-1$ & 41 \\
\hline MMP-7 & AuNP@DSNB & SERS & NA & $2.28 \mathrm{pg} \mathrm{mL}^{-1}$ & 45 \\
\hline MMP-9 & Ag@DTNB & SERS & $0 \mathrm{pg} \mathrm{mL} \mathrm{m}^{-1}-40 \mathrm{ng} \mathrm{mL}^{-1}$ & $1 \mathrm{pg} \mathrm{\textrm {mL } ^ { - 1 }}$ & 46 \\
\hline
\end{tabular}


A
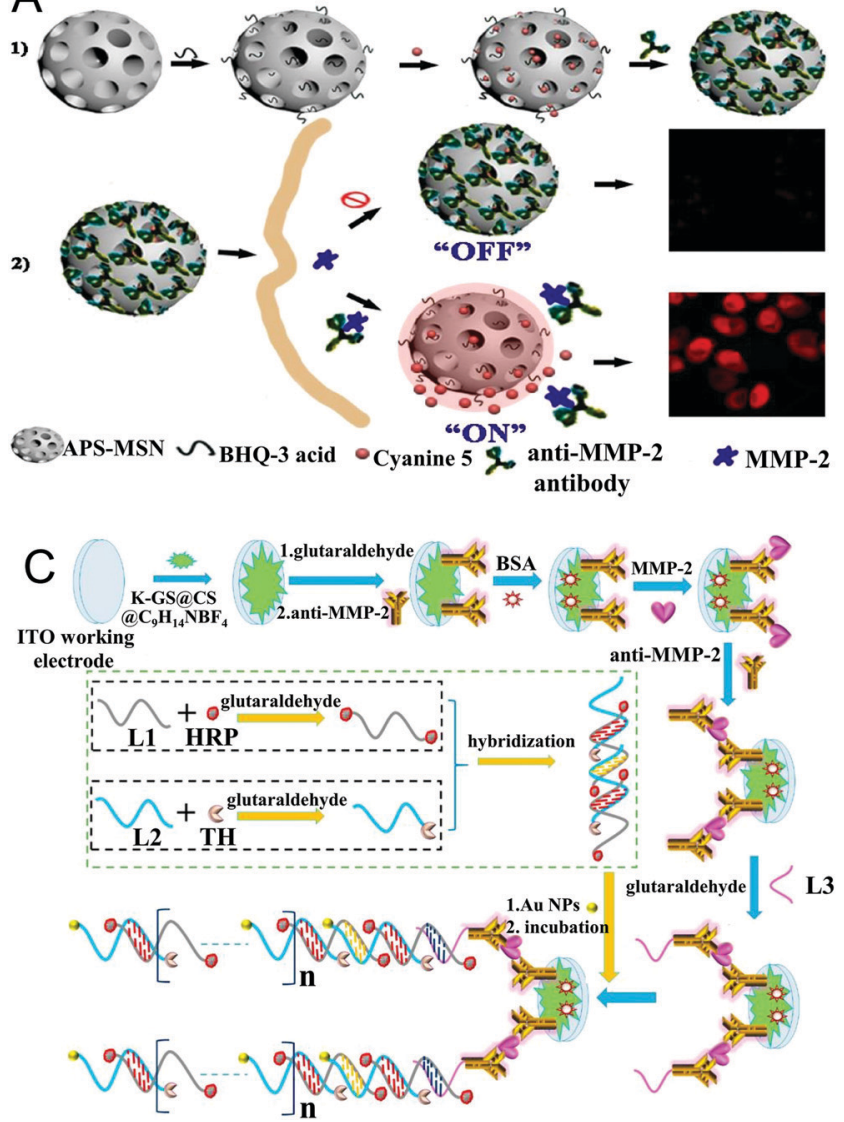
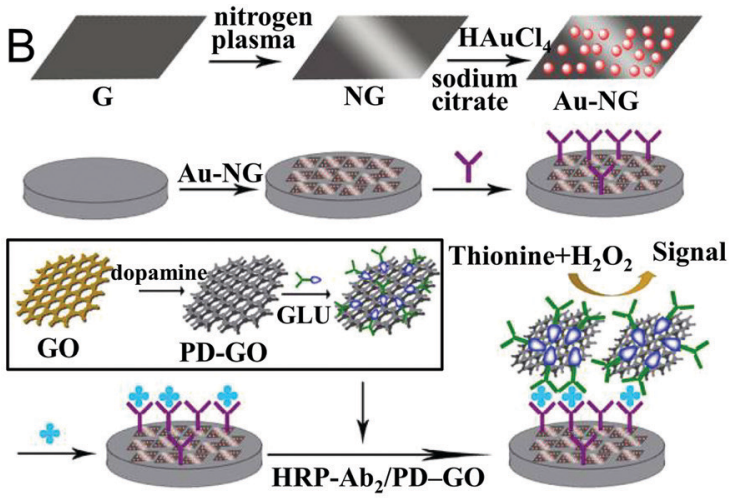

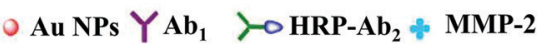

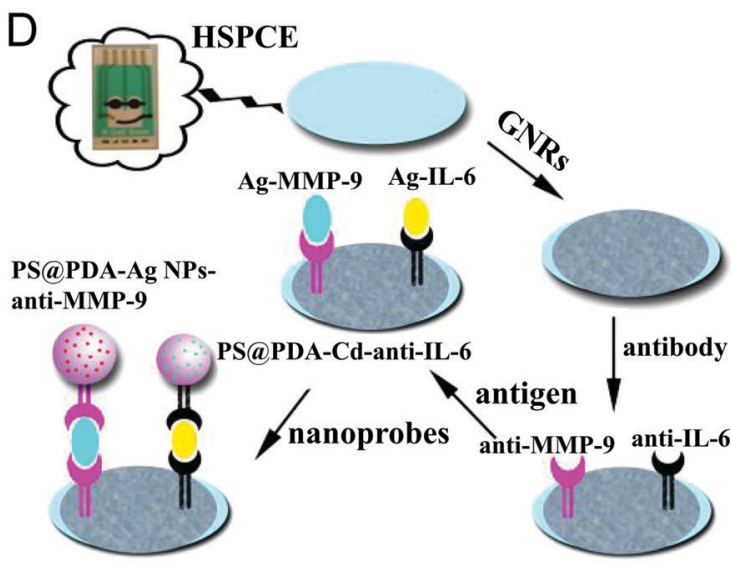

Fig. 2 (A) Illustration of the MSN-based programmed microcapsule-type MMP-2 responsive nanosensor for in situ monitoring of intracellular MMP-2 via fluorescence imaging (reprinted with permission from ref. 26. Copyright 2018, Elsevier). (B) Scheme of the electrochemical immunosensor for MMP-2 detection based on the dual signal amplification of the Au-NG composite and HRP-Ab $/$ PDA-GO bioconjugate (reprinted with permission from ref. 30 . Copyright 2013, Elsevier). (C) Schematic diagram of a super-labeled electrochemical immunosensor for MMP-2 detection with a K-GS matrix and AuNP, HRP and thionine (Th) modified aptamer labels (reprinted with permission from ref. 32. Copyright 2017, Elsevier). (D) The ultrasensitive multiplex electrochemical immunoassay based on GNR modified HSPCEs and PS@PDA-metal labels (reprinted with permission from ref. 34. Copyright 2014, Elsevier).

immunosensors, Zhang et al. developed a programmed microcapsule-type MMP-2 responsive nanosensor for in situ monitoring of intracellular MMP-2 via fluorescence imaging. ${ }^{26}$ Mesoporous silica nanoparticles (MSN) were the key components of the nanosensor, which possessed distinct advantages, such as large pore volume and surface area, good biocompatibility and easy functionalization. As shown in Fig. 2A, the microcapsule-type nanosensor was constructed by covalently coupling Black Hole Quencher 3 (BHQ-3) onto the inner walls of the mesopores of amine functionalized MSN, and then encapsulating fluorescent dye Cy5 into the mesopores to produce Cy5@MSN-BHQ, which was further sealed by anti-MMP-2 antibodies. In the absence of MMP-2, anti-MMP-2 antibodies acted as effective bio-lids to block the mesopores, thus the fluorescence of Cy5 inside the pores was quenched by the immobilized BHQ-3, resulting in an "off" state. In the presence of the target, anti-MMP-2 antibodies were detached from the MSN because of the specific binding with MMP-2, accompanied by the release of Cy5 and enhancement of red fluorescence. The "off-on" nanosensor was proportionally responsive to the MMP-2 concentration. Owing to the low cytotoxicity of the MSN-based microcapsule-type nanosensor, the intracellular MMP-2 level could be well monitored in situ by fluorescence imaging.

2.3.2 Electrochemical immunoassay. Electrochemical immunoassays have gained growing interest in analysis of protein biomarkers due to their intrinsic advantages, such as high sensitivity, low cost and easy operation. They convert the biorecognition events into measurable electrochemical signals including current, potential and impedance. As a simple and label-free technique, electrochemical impedance spectroscopy (EIS) directly measures the changes on the electrode surface, and has been applied for MMP-9 detection. ${ }^{27}$ However, due to the nonspecific adsorption, the sensitivity of ESI-based assays needs to be improved. Recently, novel technologies employing nanomaterials, such as MB, AuNPs, graphene, quantum dots (QDs) and carbon nanotubes (CNT), have been developed to improve the performance of electrochemical immunosensors for MMP detection. Moreover, the sensitivity of the assays can be further improved by various signal amplification strategies, such as multi-label signal amplifiers. Similar to the magneto-ELISA, ${ }^{24}$ 
by measuring the reduction current, Baldrich's group proposed a series of electrochemical immunoassays for simple and fast detection of MMP-9 in plasma samples using MBs as a matrix for antibody immobilization and poly-HRP as a signal enhancer. $^{28,29}$ The optimized electrochemical ELISA could be accomplished in $45 \mathrm{~min}$ with a detection limit of $18-28 \mathrm{pg} \mathrm{mL}^{-1}$. Aiming at POC testing, they developed an even faster single-step electrochemical magneto-immunoassay including only $5 \mathrm{~min}$ incubation with c-MAb modified MBs, the MMP-9-containing sample and bd-PAb/poly-HRP conjugates. ${ }^{29}$ With a customized multiplexed magnetic holder for electrochemical detection, MMP-9 detection was completed in only 12-15 min with a linear range from 0.03 to $2 \mathrm{ng} \mathrm{mL}{ }^{-1}$ and a detection limit of $13 \mathrm{pg} \mathrm{mL}^{-1}$, and the simplified electrochemical immunoassay performed well in MMP-9 quantification in clinical plasma samples.

Similar to poly-HRP, multiple enzyme loaded nanoprobes have also been used as signal amplifiers for MMP detection, which significantly increase the amount of enzyme labels per binding event. Various nanomaterials have been reported to achieve this purpose, such as graphene oxide (GO), ${ }^{30}$ and polymeric particles. ${ }^{31}$ Yang et al. reported the use of multiHRP labeled GO for ultrasensitive electrochemical analysis of MMP-2. ${ }^{30}$ As exhibited in Fig. 2B, AuNPs were assembled onto nitrogen-doped graphene (NG), and the formed composites (Au-NG) were applied to a glassy carbon electrode (GCE) as a matrix for immobilization of primary antibodies $\left(A b_{1}\right)$. The Au-NG composites not only provided a large surface area for capturing the target, but also could accelerate electron transfer and enhance the electrochemical response. HRP-labeled MMP-2 antibodies were loaded on polydopamine functionalized GO (PDA-GO); the resulting hybrids served as excellent multi-labels for signal amplification. Benefitting from the dual signal enhancement strategy, via differential pulse voltammetry (DPV) measurements, MMP-2 could be detected as low as $0.11 \mathrm{pg} \mathrm{mL}^{-1}$. In Munge's work, vertically aligned single-wall carbon nanotube (SWCNT) arrays were employed as a matrix for attachment of $\mathrm{Ab}_{1} .500 \mathrm{~nm}$ polystyrene beads conjugated with secondary antibody $\left(\mathrm{Ab}_{2}\right)$ and multiple HRP were involved for multi-label amplification. ${ }^{31}$ Compared with conventional HRP labeled $\mathrm{Ab}_{2}$ conjugates which possessed only 14-16 HRP per $\mathrm{Ab}_{2}$, the number of active HRP loaded on one polybead was estimated to be 4168. Thus, the SWCNT-based immunosensor with $\mathrm{Ab}_{2}$-polybead-HRP bioconjugates was capable of detecting as low as $4 \mathrm{pg} \mathrm{mL}^{-1} \mathrm{MMP}-3$, resulting in 55-fold higher sensitivity than the immunosensor with $\mathrm{Ab}_{2}-\mathrm{HRP}$. In addition to nanomaterials, biomolecules could also serve as HRP carriers, such as aptamers. Ren et al. presented a super-labeled electrochemical immunosensor for MMP-2 detection with a $\mathrm{K}$ doped graphene sheet (K-GS) matrix and AuNP, HRP and thionine (Th) modified aptamer labels. ${ }^{32}$ As displayed in Fig. 2C, because of its large specific surface area and excellent conductivity, the synthesized K-GS (K-GS@CS@ $\mathrm{C}_{9} \mathrm{H}_{14} \mathrm{NBF}_{4}$ ) was dropped on an ITO working electrode for immobilization of capture antibodies, which showed a better electrochemical response than GO and N-doped GS. MMP-2 was captured and detected via a sandwich immunoassay; the detection antibodies were modified with L3, and then coupled to the aptamer labels (L1@HRP-AuNP@L2@Th) for further signal amplification. The abundant AuNPs and HRP on the labels effectively catalyzed $\mathrm{H}_{2} \mathrm{O}_{2}$, while Th could accelerate the electron transportation, resulting in a triple amplification of current. Benefiting from the superior properties of K-GS and the tri infinite amplification strategy, the immunosensor showed a low detection limit of $35 \mathrm{fg} \mathrm{mL} \mathrm{m}^{-1}$ for MMP-2, and exhibited good selectivity, stability and reproducibility.

However, some disadvantages are noted about enzymebased signal amplification, such as leakage, ease of denaturation and costly preparation. Alternatively, nanomaterials with excellent electrochemical activity could be employed as enzyme-free labels. In particular, multilabel amplification can be easily achieved through assembly of the electrochemically active nanomaterials on the surface of a nanocarrier. For instance, Zhu's group developed several electrochemically active nanocomposites for MMP detection by loading CdTe QDs or metal nanoparticles (such as silver nanoparticles (AgNPs)) on the surface of polystyrene@polydopamine (PS@PDA) core@shell nanoparticles. $^{33,34}$ As shown in Fig. 2D, a sandwich electrochemical immunoassay for multiplex analysis was conducted on a heated screen-printed carbon electrode (HSPCE) by using PS@PDA/AgNP and PS@PDA/Cd ${ }^{2+}$ nanocomposites as electrochemically active labels. ${ }^{34}$ The nanocomposite-based biosensor allowed simultaneous detection of MMP-9 and interleukin-6 (IL-6) via square wave voltammetry (SWV) analysis. Benefiting from the dual amplification effect of the HSPCE technique and multilabels, as low as $5 \mathrm{fg} \mathrm{mL}^{-1}$ MMP-9 can be selectively detected.

Compared with conventional electrochemical methods, photoelectrochemical immunoassays exhibit higher sensitivity and selectivity, due to the significantly reduced background signals originating from the totally separated excitation source and detection channel. Fan et al. presented a photoelectrochemical immunosensor for ultrasensitively detecting MMP-2 on a $\mathrm{TiO}_{2}-\mathrm{NT} / \mathrm{CdS}: \mathrm{Mn} / \mathrm{CdTe}$ cosensitized structure modified electrode with the signal amplification of $\mathrm{SiO}_{2} @ \mathrm{Ab}_{2}$ conjugates. ${ }^{35}$ The cascade cosensitized structure could sufficiently absorb light energy, significantly accelerate electron transfer, and effectively prevent electron-hole recombination, thus the sensing electrode displayed significantly enhanced photocurrent intensity. In the presence of the target, specific binding of MMP- 2 with $A b_{1}$ on the electrode surface and $\mathrm{SiO}_{2} @ \mathrm{Ab}_{2}$ conjugates dramatically decreased the photocurrent intensity. Thanks to the synergetic effect of the $\mathrm{TiO}_{2}-\mathrm{NT} / \mathrm{CdS}: \mathrm{Mn} / \mathrm{CdTe}$ cosensitized structure and $\mathrm{SiO}_{2} @ \mathrm{Ab}_{2}$ conjugates, the photoelectrochemical immunosensor displayed an ultralow detection limit of $3.6 \mathrm{fg} \mathrm{mL}^{-1}$ for MMP-2.

2.3.3 SPR-based immunoassay. As a label-free optical sensing technique, SPR can provide a real-time response to the binding events on the surface of sensors. In SPR-based immunoassays, recognition molecules are immobilized on a metal surface and interact with the analytes of interest in solution, causing changes in the mass and refractive index (RI) close to the sensor surface. The changes can be quantitatively determined by measuring SPR signal shifts, such as the resonance angle, intensity or wavelength. ${ }^{36}$ Additionally, reaction kinetics can also be monitored by SPR with high 

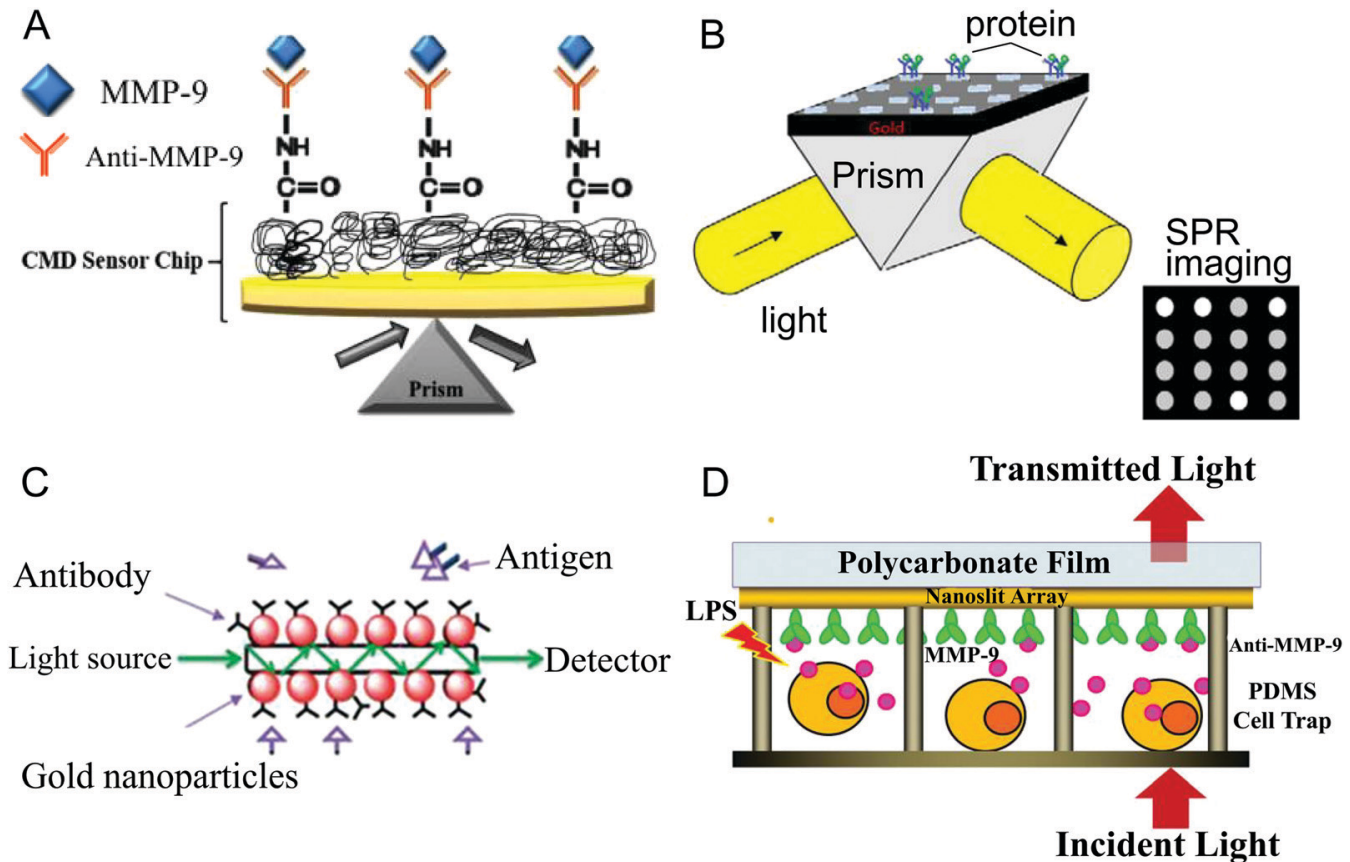

Fig. 3 SPR-based immunoassays: (A) an SPR chip with the classical Kretschmann configuration, in which monoclonal MMP-9 antibodies were immobilized on the three-dimensional CMD $50 \mathrm{D}$ chip surface for binding with the target MMP (reprinted with permission from ref. 38. Copyright 2016, Elsevier). (B) Illustration of SPRI-based immunosensors (reprinted with permission from ref. 36. Copyright 2012, Wiley-VCH). (C) FOPPR sensor chip for detecting MMPs based on the LSPR of AuNPs coated on the unclad portion of an optical fiber (reprinted with permission from ref. 41. Copyright 2013, Royal Society of Chemistry). (D) Schematic diagram of the optofluidic platform based on the Fano resonance of a gold nanoslit array for real-time and label-free determination of cell-secreted MMP-9 (reprinted with permission from ref. 43. Copyright 2013, Wiley-VCH).

sensitivity. As exhibited in Fig. 3, a variety of methodologies have been reported to build SPR-based biosensors, such as the classical Kretschmann configuration, ${ }^{37,38}$ SPR imaging (SPRI), ${ }^{39,40}$ a fiber-optic particle plasmon resonance (FOPPR) biosensor ${ }^{41,42}$ and a Fano resonance-based gold nanoslit array. ${ }^{43}$

An early study of Bolduc et al. involved 3-MPA capped binary patterned peptides as an ultralow fouling monolayer for antibody immobilization, which could effectively resist non-specific adsorption. The as-proposed SPR biosensor allowed direct determination of MMP-3 in crude bovine serum without $\mathrm{Ab}_{2}$ binding and signal amplification. ${ }^{37}$ More recently, Mohseni et al. immobilized monoclonal MMP-9 antibodies on a carboxymethyldextran hydrogel (CMD $50 \mathrm{D}$ ) chip surface for SPR measurement. ${ }^{38}$ As shown in Fig. 3A, the three-dimensional matrix of the CMD 50 D chip accommodated a good deal of antibodies, leading to dramatic signal amplification. Consequently, MMP-9 could be detected as low as $8 \mathrm{pg} \mathrm{mL} \mathrm{m}^{-1}$, and MMP-9 in saliva samples was well quantified. In traditional SPR, either the wavelength or angle of the incident beam is fixed, and the reflected light is monitored by a single-element detector. When both the wavelength and angle are fixed, the specific binding of the antibody and antigen could be detected by SPRI, which measures the reflectance change across the sensing surface by a charge coupled device (CCD) camera (as shown in Fig. 3B). Moreover, SPRI sensors can be prepared into arrays, and allow parallel monitoring of numerous reactions in real time. Tokarzewicz et al. proposed an SPRI-based biosensor for quantitative determination of MMP-1 by immobilizing an antibody on a cysteamine monolayer coated chip as a receptor. ${ }^{39}$ The detection limit of MMP-1 was $9 \mathrm{pg} \mathrm{mL}^{-1}$, and good results were obtained in real blood plasma analysis.

Classical prism-based SPR sensors need bulky metal films and costly optical components, while fiber-optic based SPR sensors circumvent these issues with a metal NP coated optical fiber as a matrix for immobilization of the receptor, resulting in high sensitivity. In addition, the FOPPR sensors based on the localized surface plasmon resonance (LSPR) of metal NPs simply measure optical extinction. ${ }^{41,42}$ As displayed in Fig. 3C, the unclad portion of an optical fiber was firstly modified with AuNPs, and then a monolayer of a linker was self-assembled on the AuNP surface for immobilization of antibodies. An evanescent wave was applied to the optical fiber, and propagated via multiple total internal reflection (TIR), thus the light was attenuated by interacting with the AuNP monolayer. After binding with the target, the surrounding RI and the absorption coefficient of the AuNPs were increased. The change of the transmitted light intensity was in a good linear relationship with the MMP-3 concentration in the range of $0.05-50 \mathrm{ng} \mathrm{mL} \mathrm{m}^{-1}$. Integrated with a microfluidics device, the FOPPR biosensor achieved a detection limit of $34 \mathrm{pg} \mathrm{mL}{ }^{-1}$ for MMP-3. ${ }^{41}$

The popular SPR sensors are designed on the basis of either SPR of metal films or LSPR of metal NPs, while nanostructure array-based sensors have gained popularity for detecting biomolecules. Wu et al. constructed an optofluidic platform for real-time detection of cell-secreted MMP-9 by integrating a gold nanoslit array-based SPR biosensor with a microfluidic device. ${ }^{43}$ 
Large-area gold nanoslit arrays were fabricated on polycarbonate films, exhibiting both SPR and LSPR modes. The constructive and destructive interferences of LSPR and Bloch wave SPP caused an asymmetrical Fano resonance. The intensity sensitivity of the Fano resonance $\left(\sim 1.15 \times 10^{4} \%\right.$ per RIU) was markedly higher than that of prism-based SPR biosensors ( $\sim 3900 \%$ per RIU), and could be further improved to $6.22 \times 10^{5} \%$ per RIU by integrating signals from the whole responsive range. Therefore, the integrated intensity change was used for monitoring surface binding events. As shown in Fig. 3D, specific binding of MMP-9 with antibodies on the nanoslit array increased the surface RI, causing a concentration-dependent integrated response. Monocytic leukemia THP1 cells were trapped into the U-shaped cell trap arrays, and MMP-9 secretion from cells treated with or without LPS stimulation was monitored in real time by measuring the transmitted light intensity. Due to the ultrahigh sensitivity of the sensing system, MMP-9 secreted by as few as 2 cells with LPS stimulation could be detected, which is much better than conventional ELISA.

2.3.4 Surface-enhanced Raman scattering (SERS)-based immunoassay. SERS is another optical technique measuring the Raman scattering signal, which can be enhanced by a roughened metal surface. Due to the strong SPR of metal nanostructures, Raman signals of reporter molecules assembled on the surface can be enhanced up to $10^{4}-10^{8}$ fold compared with normal Raman scattering. ${ }^{44}$ To achieve this goal, $\mathrm{Au}$ and $\mathrm{Ag}$ are top priorities; the hot spots in the nanoscale junctions or interstices of metal nanostructures generate intense electromagnetic fields, leading to significant enhancement of Raman scattering. Raman spectra show characteristic fingerprint spectra of reporter molecules, endowing SERS assays with high specificity. In addition, the narrow spectral widths of Raman peaks make them easy to separate and identify. Therefore, combined with different Raman labels, SERS shows great potential for multiplex analysis. Granger et al. developed an AuNP-based SERS multiplexed platform for simultaneously detecting serum carbohydrate antigen 19-9 (CA 19-9) and MMP-7. ${ }^{45}$ Anti-MMP-7 and anti-CA 19-9 antibodies were immobilized on designated sites of a gold array to prepare the capture substrate. AuNPs loaded with 5,5'-dithiobis(succinimidyl-2nitrobenzoate) (DSNB) and antibodies served as extrinsic Raman labels (ERLs) for detection, which showed a Raman signal at $1336 \mathrm{~cm}^{-1}$. The detection limits were $2.28 \mathrm{pg} \mathrm{mL}^{-1}$ for MMP-7 and $34.5 \mathrm{pg} \mathrm{mL}^{-1}$ for CA 19-9. The results of analysing MMP-7 in diluted serum samples were comparable to ELISA, while the sample consumption was ten-time less than ELISA. Zhao et al. proposed an immunoassay for detecting MMP-9 in unprocessed blood samples, which integrated SERS nano-tags with a magnetic separation strategy. ${ }^{46}$ The SERS nano-tags were prepared by adsorbing a sub-monolayer of 5,5'-dithiobis-(2nitrobenzoic acid) (DTNB) on polyvinylpyrrolidone (PVP) modified AgNPs, which were more stable for biological analysis. The resultant Ag@DTNB nano-tags were conjugated with MMP-9 monoclonal antibodies for detection. $\mathrm{Fe}_{3} \mathrm{O}_{4}$ microspheres functionalized with MMP-9 polyclonal antibodies were employed for capture and magnetic separation. Based on the sandwich immunoassay, the Raman signals of DTNB at $1332 \mathrm{~cm}^{-1}$ were recorded for quantification of MMP-9, which were proportional to the MMP-9 concentration in the low and high range. Thanks to the Ag@DTNB SERS nano-tags, the detection limit was $1 \mathrm{pg} \mathrm{mL} \mathrm{m}^{-1}$ for MMP-9, which was much lower than the commercial kit (50 pg $\left.\mathrm{mL}^{-1}\right)$. Due to the sensitivity and simplicity of the assay, MMP-9 in human whole blood samples could be determined rapidly with a portable Raman spectrometer.

\subsection{Immunocapture assay}

Since conventional immunoassays depend on the recognition of epitopes, all forms of MMPs could be determined, including latent MMPs, active MMPs and MMP-TIMP complexes. They provide information about the total level of a specific MMP rather than the activity of the MMP, which really matters in body function. In 1997, Verheijen et al. firstly described a simple immune recognition-based colorimetric assay for determining MMP proteolytic activities, ${ }^{47}$ which was further developed into an immunocapture assay. The assay is based on the recognition of modified pro-urokinase by active MMPs. To be specific, by protein engineering, the activation site in pro-urokinase normally recognized by plasmin (Pro-Arg-PheLys-Ile-Ile-Gly-Gly) is replaced with a sequence that is specifically recognized by MMPs (Arg-Pro-Leu-Gly-Ile-Ile-Gly-Gly). Upon activation by MMPs, the active urokinase (UKCOL) is assayed with a chromogenic peptide substrate. After being captured from biological fluids by the immobilized specific antibodies, the active MMPs are analysed directly by addition of the modified pro-urokinase and peptide substrate, while latent MMPs need to be firstly activated by APMA. MMP activities are determined by monitoring the change of absorbance over time. In contrast to ELISA-based assays, immunocapture assays are capable of quantifying active MMPs as well as pro-MMPs, and are already commercially available as kits.

Considering the complexity of protein engineering and enzyme cascade, the urokinase-related assay was later replaced with a one-step enzymatic proteolysis reaction by directly adding fluorogenic peptide substrates. The peptide substrates containing a fluorescence resonance energy transfer (FRET) acceptor/donor pair are hydrolyzed by the captured MMPs, resulting in the recovery of fluorescence. MMP activities are determined by monitoring the changes of fluorescence intensity. However, MMP activities may be lost if their active sites are obscured by the capture antibodies, hence antibody recognition has a significant influence on the sensitivity of the assay. In order to achieve the maximum MMP immunocapture efficiency, Hawkins et al. modified the assay by firstly coating the plate with protein $\mathrm{G}$ to correctly immobilize and orient the capture antibody. ${ }^{48}$ Protein $\mathrm{G}$ bound to the Fc region of the antibody, leaving the Fab region in an optimal orientation for capturing MMP-9. Commercially available FRET peptide substrates with 5 -FAM/QXL ${ }^{\mathrm{TM}} 520$ as a donor/acceptor pair were used for determining MMP-9 activity. The total and endogenously active MMP-9 in multiple biological samples were analysed with high sensitivity and specificity. 


\section{Biosensors for measuring MMP activities}

Although immunoassays have been extensively used for detecting MMPs, they mostly measure the total amount of MMP protein, and are unable to distinguish pro-MMPs and active MMPs. In fact, MMPs play their roles by executing their proteolytic function. Dysregulation of the MMP activity may cause severe disease, thus determination of actual MMP activities is more meaningful. So far, a variety of assays for analysing MMP activity have been developed, such as zymography, biosensors and microfluidics-based platforms. In particular, numerous multifunctional nanoprobes have been employed for real-time and in vivo monitoring of MMP activities.

\subsection{Zymography}

Zymography is a gel electrophoresis-based method for analysing the proteolytic activity of MMPs. Specifically, MMPs' protein substrates (gelatin, casein or collagen) are copolymerized with acrylamide and sodium dodecyl sulfate (SDS), forming an SDSpolyacrylamide gel. Because of the difference in molecular weight, MMPs sharing the same substrate can be separated simultaneously on a single gel by nonreducing SDS-PAGE. Active MMPs are denatured during electrophoresis due to the reversible binding with SDS. After electrophoresis, the MMPs partially recover their activities by the exchange of SDS with non-ionic detergent (e.g., Triton X-100) in the washing buffer. Meanwhile, the pro-MMPs are autoactivated during the renaturation process. Subsequently, the renatured MMPs in the gel digest the local substrates when incubated with $\mathrm{Ca}^{2+}$ and $\mathrm{Zn}^{2+}$-containing buffer. Finally, the gel is stained with Coomassie blue, and the MMPs are evidenced as clear bands against the blue background. MMP activities can be quantitatively determined by analysing the intensity of the clear bands with densitometry. ${ }^{2}$ In addition, the MMP/TIMP complexes are disrupted by SDS during electrophoresis, and the dissociated MMPs' activities are also detected. Compared with immunoassays, latent zymogens and active MMPs can be well discriminated by different clear bands in zymography.

As the name implies, gelatin zymography is sensitive for gelatinases (MMP-2 and MMP-9), while for collagenases (MMP-1 and MMP-13), collagen zymography is preferred with native collagen fibrils as the substrate. For stromelysins (MMP-3, MMP-10 and MMP-11) and matrilysin (MMP-7), casein zymography is more suitable. ${ }^{12}$ However, the sensitivity of casein zymography is much lower than gelatin zymography. In addition, because of the relatively low molecular weight $(23 \mathrm{kDa})$, casein migrates during the electrophoresis, leading to an inhomogeneous background in the gel with two clearly defined zones. Analysis of MMPs located near the casein migration boundary would be severely affected, such as MMP-7. Pre-running the casein-containing gel before electrophoresis is an effective solution, allowing the excess casein in the lower part to run out of the gel. Whereas when MMP-1, MMP-13 and MMP-7 are present at very low levels, they are difficult to detect by conventional substrate zymography. Some variations have been made to improve the performance of conventional zymography, for instance, heparin-enhanced zymography, reverse zymography and in situ zymography. The above zymographic techniques have been thoroughly reviewed by Snoek-van Beurden et al. ${ }^{2}$ and others, ${ }^{49}$ and no more redundant description is provided here.

\subsection{Biosensors}

Although zymography exhibits superiority in detection of MMPs in complex biological samples, it is limited to a few MMPs, and the experimental procedures are rather complicated and time-consuming. Owing to the simplicity and high sensitivity, biosensors have been developed as alternatives to promote the detection of biomolecules. Generally, a biosensor is composed of a bioreceptor and a transducer: the bioreceptor is employed for specifically interacting with the target analyte, while the transducer transforms the recognition events into measurable signals. In addition, signal amplification strategies can be introduced to further enhance the sensitivity of biosensors. Recently, a variety of nanoparticle-based biosensors have achieved success in determining MMP activities. Depending on the type of sensing technique, nanoparticles can serve as effective transducers for optical biosensors, electrochemical biosensors, magnetic biosensors and photoacoustic biosensors. The assay performances of reported biosensors for determination of MMP activities are summarized in Table 2.

3.2.1 Optical biosensors. Optical biosensors provide simple, fast and low cost approaches for determination of MMP activities. According to the change in optical properties of transducers, current optical biosensors are mainly classified into the following categories: colorimetric biosensors, fluorescence biosensors, SPR biosensors and SERS biosensors. In particular, we will highlight the recent advancement in nanomaterial-based optical biosensors for MMP detection.

3.2.1.1 Colorimetric biosensors. Colorimetry-based biosensors measure the absorption of a solution undergoing a color change during the recognition response, which can be achieved only by a spectrophotometer. Due to its simplicity, colorimetry is prevalent for analysing different kinds of targets, and the visualized output shows great promise for POC testing. Like ELISA, enzyme-catalyzed chromogenic reactions have also been employed to construct colorimetric biosensors for determination of MMP activities. ${ }^{50,51}$ Banerjee et al. developed a liposome-mediated colorimetric biosensor with an HRP-based signal amplification strategy for detecting cell-secreted MMP-9. ${ }^{50}$ As shown in Fig. 4A, HRP was encapsulated into liposomes, which were composed of an MMP-9 lipopeptide substrate (GPO4) with triple helical conformation. O-Phenylenediamine (OPD) and $\mathrm{H}_{2} \mathrm{O}_{2}$ serving as substrates for HRP were added into the MMP-9 containing buffer for signal reporting and amplification. When liposomes were recognized by MMP-9, the degradation of GPO4 caused the disintegration of the liposomes, making HRP leak out and make contact with its substrates. HRP-catalyzed oxidation of OPD formed a colored product, and the MMP-9 activity was determined by measuring the absorbance of the system. The assay displayed good specificity and a wide linear range, covering the MMP-9 level in normal tissues $\left(10 \mathrm{nmol} \mathrm{L}^{-1}\right)$ and cancerous tissues 
Table 2 Assay performance of reported biosensors for determination of MMP activities (NA: not available)

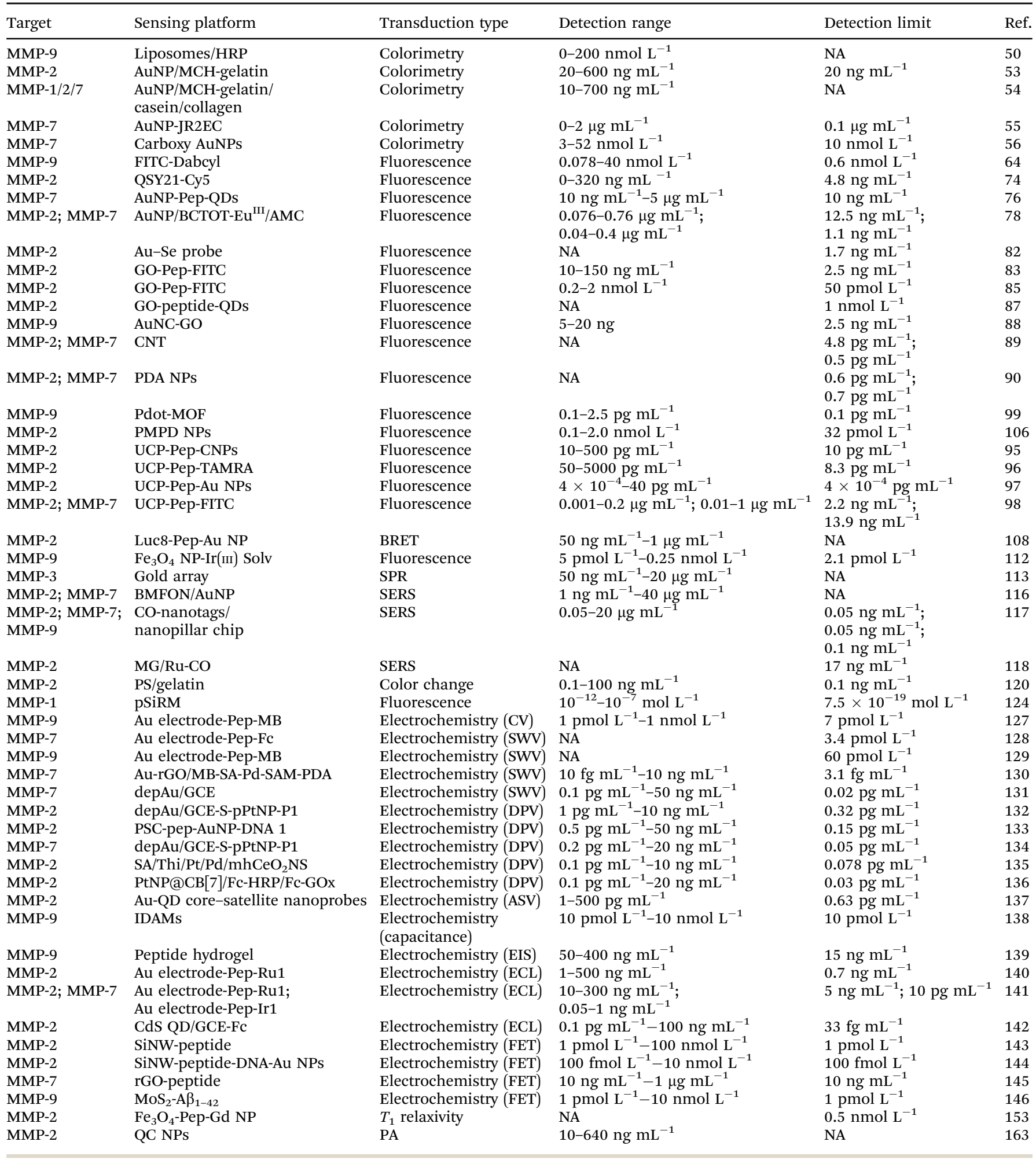

(100-200 $\mathrm{nmol} \mathrm{L}^{-1}$ ), and was employed to estimate the concentration of MMP-9 secreted from MCF-7 and HT-29 cells.

Besides catalytic chromogenic reaction, AuNPs are popular in colorimetric assay as signal reporters. The strong LSPR of AuNPs endows them with characteristic light absorption and scattering, which are greatly dependent on their shape, size and aggregation state. Generally, monodisperse AuNPs are red in color with a distinctive SPR peak; when they are aggregated, the size of the AuNPs increases, accompanied by a red shift of the SPR peak and a color change from red to purple-blue. ${ }^{52}$ 

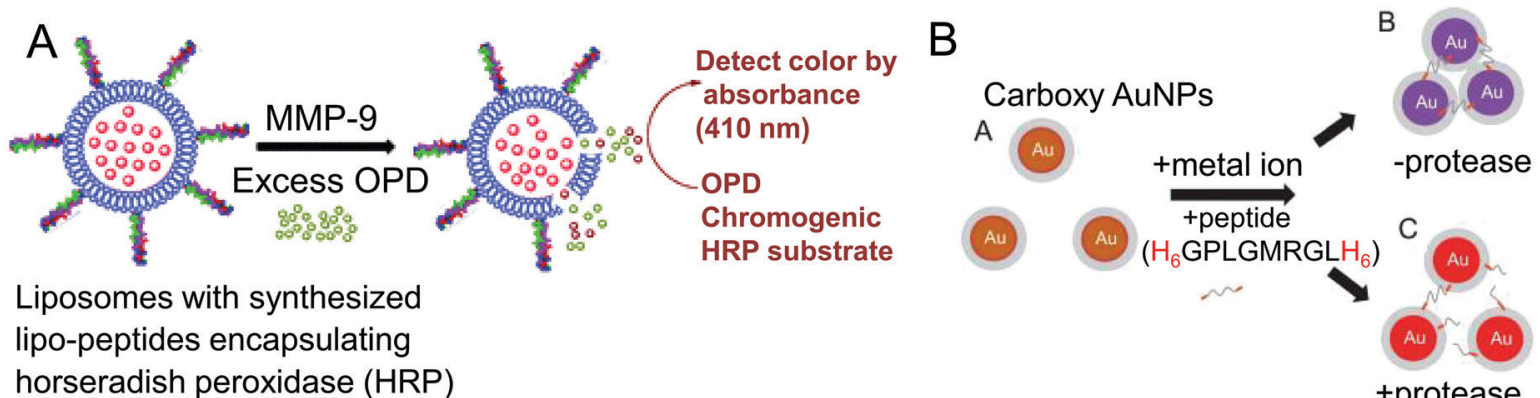

lipo-peptides encapsulating
horseradish peroxidase (HRP)

+protease

\section{C}

A

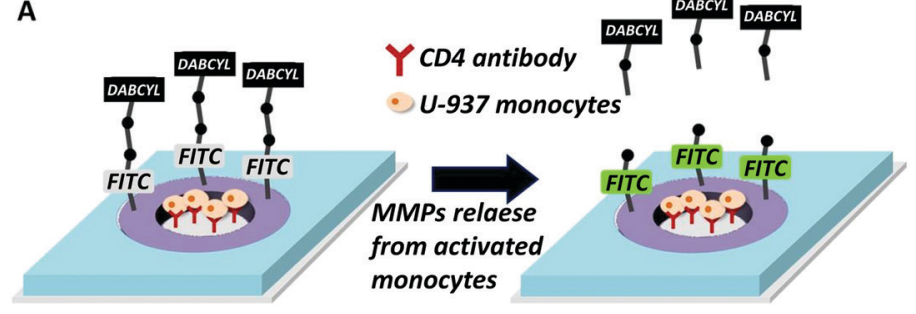

B
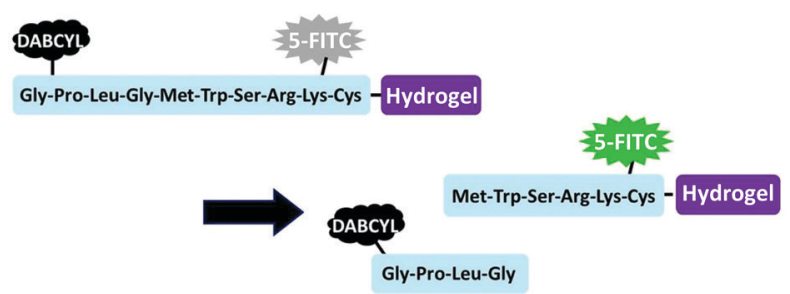

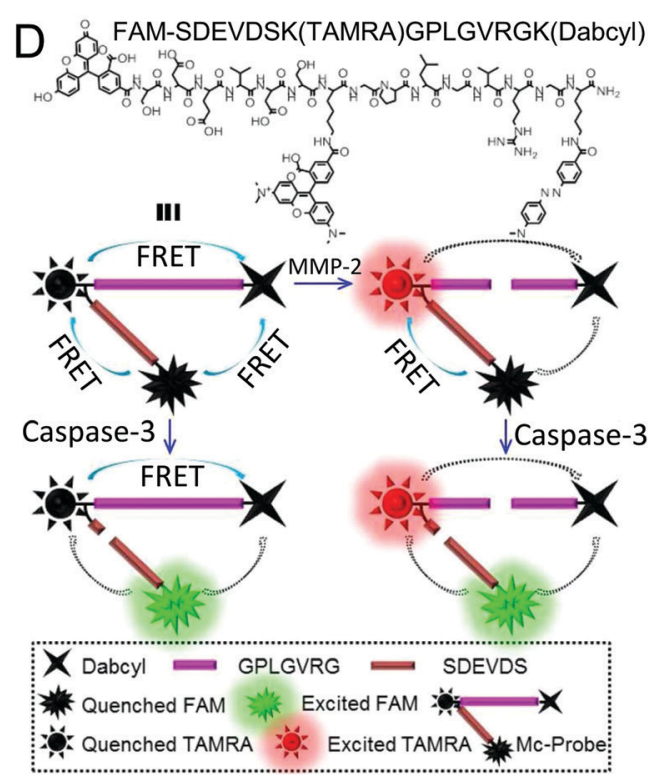

Fig. 4 (A) Schematic plot of the liposome-based colorimetric biosensor for determination of MMP-9 activities (reprinted with permission from ref. 50. Copyright 2010, Royal Society of Chemistry). (B) Colorimetric assay for MMP-7 activity detection based on self-assembly of carboxy AuNPs and $\mathrm{H}_{6}$ tagged peptides (reprinted with permission from ref. 56. Copyright 2013, Elsevier). (C) Micropatterned hydrogels modified with specific FRET peptide substrates for sensing MMP-9 secreted from cells trapped in the hydrogel microwells (reprinted with permission from ref. 64. Copyright 2013, American Chemical Society). (D) Chemical structure and detection principle of the multi-FRET based fluorescent probe for imaging MMP-2 and Caspase-3 (reprinted with permission from ref. 66. Copyright 2017, American Chemical Society).

The color change in appearance is the basis of AuNP-based colorimetry, and can be quantified by measuring the absorbance at the SPR peak. Chuang et al. established an AuNPbased optical biosensor for determination of MMP-2 activity, in which $13 \mathrm{~nm}$ AuNPs were modified with gelatin as the MMP-2 substrate and functionalized with 6-mercaptohexan-1-ol (MCH) as blocker and inducer. ${ }^{53}$ After being treated with MMP-2, the gelatin on the AuNP surface was degraded, while $\mathrm{MCH}$ prevented the digested peptide fragments from adsorbing on the AuNPs, leading to the aggregation of the AuNPs. The color of the solution changed from red to purple, which was evidenced by a decrease of absorbance at $525 \mathrm{~nm}$ and an increase of absorbance at $625 \mathrm{~nm}$. The ratio of the absorbance at $625 \mathrm{~nm}$ and $525 \mathrm{~nm}\left(A_{625} / A_{525}\right)$ was proportional to the MMP-2 concentration in the range from $20 \mathrm{ng} \mathrm{mL}^{-1}$ to $600 \mathrm{ng} \mathrm{mL} \mathrm{mL}^{-1}$. By changing the substrate on the AuNPs, the colorimetric biosensor was extended to other MMPs (MMP-1, MMP-2 and MMP-7), and was used to determine specific MMP activity in plasma samples. ${ }^{54}$ Although the assay is superior in easy operation and short assay time, the modification of protein macromolecules sacrifices the sensitivity of AuNP-based colorimetric biosensors, moreover, the protein normally lacks stability. With the development of degradomics and proteomics, a number of short peptides have been identified as specific MMP substrates. Thus, many smart biosensors could be designed with peptides as recognition receptors for determination of MMP activities. Based on MMP-induced aggregation of AuNPs, Chen et al. developed a colorimetric biosensor for MMP-7 detection with a peptide substrate (JR2EC) containing two cleavage sites as a receptor. ${ }^{55}$ In contrast, Kim et al. proposed a reverse colorimetric biosensor for detecting MMP-7 activity via the transformation of AuNPs from aggregation to dispersion. ${ }^{56}$ As displayed in Fig. 4B, carboxy AuNPs were linked by peptides containing MMP-7 cleavage sites and two hexahistidine $\left(\mathrm{H}_{6}\right)$ tails at both terminals via metal-affinity coordination, forming clusters in a violet color. In the presence of MMP-7, the peptides were cleaved and the aggregation of the AuNPs was prevented, hence the solution maintained the original reddishbrown color of carboxy AuNPs. Under the optimal experimental conditions, the normalized extinction ratio was well linear with 
the MMP-7 concentration in the range of $3 \mathrm{nmol} \mathrm{L}^{-1}$ to $52 \mathrm{nmol} \mathrm{L}^{-1}$ with a detection limit of $10 \mathrm{nmol} \mathrm{L^{-1 }}$.

3.2.1.2 Fluorescence biosensors. Compared with colorimetry, fluorescence biosensors are more widely used in protease detection because of their high sensitivity, good selectivity and multiplex detection ability. The fluorescence biosensors for MMP detection are mainly designed on the basis of fluorescence self-quenching, ${ }^{57,58}$ FRET $^{59-106}$ or bioluminescence resonance energy transfer (BRET). ${ }^{107-111}$ FRET-based biosensors spring up, and have become the most popular approaches for determination of MMP activities. FRET-based biosensors are typically comprised of peptide substrates flanked by a fluorescence donor and acceptor in close proximity of 1-10 $\mathrm{nm}$. When the fluorescence emission spectrum of the donor overlaps with the absorption spectrum of the acceptor, energy transfer from the donor to acceptor occurs in a nonradiative manner, thus the fluorescence of the donor is quenched by the acceptor. After being exposed to MMPs, the cleavage of the peptide substrate leads to the separation of the donor-acceptor pair and termination of the FRET process, accompanied by the fluorescence recovery of the donor. With the advancement of materials science, in addition to traditional organic fluorescent dyes, various kinds of nanomaterials have been employed as FRET donors or acceptors.

(1) Organic fluorescent dyes as FRET donors/acceptors. The commonly used organic fluorescent dyes could emit light covering all wavelength ranges, including the ultraviolet (UV), visible and near infrared regions (NIR). The fluorophores with UV emission are typically coumarin and naphthalene-based structures, such as 7-methoxycoumarin (Mca)/2,4-dinitrophenyl (Dnp) ${ }^{59,60}$ 5-((2-aminoethyl)amino)-naphtha-lene-1-sulfonic acid (EDANS)/4-(4-methylaminophenylazo)benzoyl (Dabcyl), ${ }^{61}$ and ortho-aminobenzoyl (Abz)/N-(2,4-dinitrophenyl)ethylenediamine (EDDnp). ${ }^{62}$ Hai et al. proposed a capillary electrophoresis-based fluorescence sensing system for high-throughput screening of MMP inhibitors using peptide substrates with Mca/Dnp as a FRET donor/acceptor pair. ${ }^{60}$ MMPs, peptide substrates and inhibitors were injected together into the capillary via a sandwich mode, then mixed, reacted and separated by electrophoretically mediated microanalysis (EMMA) within $70 \mathrm{~s}$. The MMP activity was determined by measuring the recovered fluorescence of Mca. The inhibition mechanism and kinetic constant $\left(K_{\mathrm{m}}\right)$ were estimated by analysing the initial velocity. The assay was capable of quantitatively evaluating the inhibition potencies of several compounds against MMP-2 and MMP-9.

For fluorochromes that emit in the visible range, Dabcyl is an ideal nonfluorescent quencher, because of its wide absorption spectrum from $360 \mathrm{~nm}$ to $560 \mathrm{~nm}$. The commonly used energy donors for Dabcyl in MMP activity analysis include FAM, FITC, fluorescein and TAMRA. ${ }^{63-66}$ Son et al. integrated a hydrogel microwell-based FRET biosensor with a reconfigurable microfluidic device for real-time detection of cell-secreted MMP-9. ${ }^{64}$ As displayed in Fig. 4C, the micropatterned PEG hydrogel microwells were modified with ligands for cell adhesion. Meanwhile, specific MMP-9 peptide substrates with FITC and
Dabcyl as a fluorophore/quencher pair were immobilized on the wall of hydrogel rings for sensing cell-secreted MMP-9 from the microwells. After being cleaved by MMP-9, the Dabcyl-containing peptide fragments were released from the hydrogel, while the peptide moieties with FITC were still retained in the hydrogel. By monitoring the fluorescence of the remaining FITC, MMP-9 could be determined as low as $0.6 \mathrm{nmol} \mathrm{L}^{-1}$. In combination with reconfigurable microfluidics, the sensitivity of the biosensor was enhanced, and MMP-9 secreted from as few as 11 cells could be detected. Interestingly, Zhang's group designed a fluorescent probe on the basis of multi-FRET processes between FAM, TAMRA and Dabcyl for simultaneously imaging MMP-2 and caspase-3. ${ }^{66}$ As shown in Fig. 4D, FAM served as the donor for both TAMRA and Dabcyl in the probe, meanwhile Dabcyl was the acceptor for both FAM and TAMRA. TAMRA was the donor for Dabcyl, but the acceptor for FAM. Initially, the fluorescence of both FAM and TAMRA was quenched via the multi-FRET processes. When incubated with MMP-2, the FRET between TAMRA and Dabcyl was terminated, along with the recovery of TAMRA fluorescence. After being recognized by caspase-3, the probe was further cleaved, leading to the separation of FAM from TAMRA, and fluorescence recovery of FAM. The recovered fluorescence intensity was linear with the MMP-2 and caspase- 3 concentration, and the sensing process of MMP-2 was independent of caspase-3. Because of the high sensitivity and specificity, the probe was able to simultaneously image MMP-2 and caspase-3 activities in cancer cell lines, demonstrating great potential for precise disease diagnosis and therapy.

Compared with fluorescent dyes with UV or visible emission, fluorochromes with longer emission in the NIR region such as Cy5 and Cy5.5 possess deeper tissue penetration, and thus are superior for in vivo imaging of MMP activities. To design FRET-based biosensors, nonfluorescent dyes, referred to as dark quenchers, are preferred, such as BHQ-3 ${ }^{67-72}$ and QSY21. ${ }^{73,74}$ The latest work reported by Yin et al. involved an MMP-2 activatable probe with Cy5 and QSY21 as a fluorophore/quencher pair. ${ }^{74}$ The probe was composed of a radionuclide $\left({ }^{125} \mathrm{I}\right)$ and a tumor-targeting peptide cRGD labeled peptide substrate, flanked by Cy5 and QSY21. The probe was optically silent in its intact state; upon being cleaved by activated MMP-2, the termination of the FRET process caused recovery of Cy5 fluorescence, which was linear with the MMP-2 concentration below $320 \mathrm{ng} \mathrm{mL}{ }^{-1}$. The MMP-2 activity in murine breast carcinoma 4T1 cells was imaged by this probe with high specificity. Moreover, due to the excellent tumor-targeting ability, the probe was further exploited to image metastatic lymph nodes (MLNs) in a 4T1-bearing mouse model. In particular, owing to the labeling with Cy5 and ${ }^{125} \mathrm{I}$, the probe allowed NIR fluorescence and single-photon emission computed tomography (SPECT) dual-modal imaging of MLNs in vivo.

(2) Nanomaterials as FRET donors/acceptors. Organic fluorescent dyes are commercially available and easy to conjugate with biomolecules, but they may suffer from short circulation times in vivo and poor photostability. With the development of advancing nanotechnology, a variety of nanomaterials with unique optical properties have been successfully used as 
donors or acceptors in FRET-based biosensors for determination of MMP activity. Up to now, AuNPs, ${ }^{75-82} \mathrm{GO},{ }^{83-88} \mathrm{CNT},{ }^{89}$ polydopamine nanoparticles (PDANPs) ${ }^{90}$ and $\mathrm{Fe}_{3} \mathrm{O}_{4}$ nanoparticles $\left(\mathrm{Fe}_{3} \mathrm{O}_{4} \mathrm{NPs}\right)^{91}$ have achieved certain success as quenchers. While QDs, ${ }^{92-94}$ upconversion nanoparticles (UCNPs), ${ }^{95-98}$ and other fluorescent nanomaterials ${ }^{99-102}$ are good candidates for fluorescence donors.

Owing to the broad absorption cross section, AuNPs are capable of quenching fluorescence emission in the visible and NIR regions. Furthermore, the dipole moments of AuNPs are not defined; energy transfer occurs near the AuNP surface regardless of the orientation to the donors, resulting in enhanced quenching efficiency. When the fluorescence donors are close to the AuNP surface, the fluorescence is quenched, while when they are detached from the AuNPs, the fluorescence is recovered..$^{103}$ Moreover, the good biocompatibility of AuNPs shows great potential for in vivo applications. Lee et al. developed a NIR fluorescence (NIRF) probe to determine MMP activities by coupling Cy5.5-labeled peptide substrates on AuNPs. ${ }^{75}$ The probe was selectively responsive to MMP-2, MMP-3, MMP-7 and MMP-13, and was successfully used for MMP sensing, inhibitor screening and early tumor detection. Tang's group designed a novel AuNP-based fluorescence probe for multiplex detection of MMP-2 and MMP-7 with a single excitation wavelength. ${ }^{78}$ The probe was prepared by assembling peptide substrates containing both MMP-2 and MMP-7 cleavage sequences onto the AuNP surface via two intermediate cysteine residues. The peptide substrates were labeled with lanthanide complex (BCTOT-Eu ${ }^{\text {III }}$ ) and 7-amino-4-methylcoumarin (AMC) on the N-terminus and C-terminus, respectively, which showed two completely resolved emission peaks. In the presence of the target MMP, the peptide substrates were partly hydrolyzed, and the corresponding FRET process was terminated, leading to the recovery of fluorescence. The fluorescence intensity was in good linearity with the MMP concentration, and the detection limits for MMP-2 and MMP-7 were $12.5 \mathrm{ng} \mathrm{mL}^{-1}$ and $1.1 \mathrm{ng} \mathrm{mL}^{-1}$, respectively. Furthermore, the nanoprobe with single excitation and multiple emissions showed high specificity for the two targeted MMPs without any cross-talk, and performed well in confocal fluorescence imaging of cells in vitro. Generally, AuNPbased biosensors are designed by assembling ligands on their surface via $\mathrm{Au}-\mathrm{S}$ covalent bonds. However, abundant biothiols in vivo would challenge this system due to competitive ligand exchange, resulting in false positives and signal distortions. To address this problem, Tang's group proposed an MMP-2 imaging probe based on Au-Se bonds, allowing sensing MMP-2 under high-thiol conditions. ${ }^{82}$ As shown in Fig. 5A, the probe was designed by conjugating selenol modified and FITC labeled peptide substrates onto the AuNP surface via Au-Se bonds, which displayed higher thermal stability and much better resistance to glutathione (GSH) than $\mathrm{Au}-\mathrm{S}$ bonds. In the presence of GSH at high concentration, the fluorescence of the $\mathrm{Au}-\mathrm{Se}$ probe was enhanced with increasing concentration of MMP-2; a higher signal to noise ratio (10.1) and lower detection limit $\left(1.7 \mathrm{ng} \mathrm{mL} \mathrm{m}^{-1}\right)$ were achieved. Intracellular MMP-2 in HepG2 cells and MCF-7 cells was well imaged by this probe, showing good application prospects in creating biosensors for in vivo detection without interference from biothiols.

As a single-atom-thick two-dimensional carbon material, GO exhibits a large absorption cross section and long-range nanoscale energy transfer property, and thus has been extensively used as an effective quencher in FRET-based MMP biosensors. In addition, GO possesses good biocompatibility and water dispersibility, and is easy to modify. Fluorophore labeled peptide substrates can be assembled on GO by covalent coupling ${ }^{83,84}$ or adsorption via $\pi-\pi$ interactions. ${ }^{85,86}$ Adsorption-based GO biosensors are easy to prepare, but they are unstable and susceptible to interference from other competitive molecules, limiting their practical application in complex samples. To prepare a robust FRET-based biosensor, Song et al. conjugated FITC-labeled peptide substrates on the GO surface through the EDC/NHS-mediated coupling reaction between primary amines of the peptide terminal and carboxyl groups of GO. ${ }^{83}$ The obtained GO-Pep-FITC FRET biosensor was more stable under physiological conditions than the one prepared via physical adsorption, and was capable of detecting MMP-2 in human serum samples with high sensitivity and good accuracy. Similarly, Yue et al. constructed an inducible probe by covalently attaching Cy5-labeled peptide substrates on nanosized GO sheets. ${ }^{84}$ Since the core peptide (PLGVR) in the substrate was responsive to various MMPs (MMP-2, -9 and -13) overexpressed in tumors, the probe was switched on in the tumor region. Due to the good biocompatibility, the probe was applied for in vivo imaging through the permeation and retention (EPR) effect to achieve early tumor diagnosis and therapeutic monitoring. Besides organic fluorescent dyes, GO is also an effective quencher for fluorescent nanomaterials, such as QDs and gold nanoclusters (AuNCs). ${ }^{87,88}$ As illustrated in Fig. 5B, Nguyen et al. presented a GO-based turn-on fluorescence probe with AuNCs as the energy donor, in which MMP peptide substrates served as co-templating ligands with mercaptoundecanoic acid (MUA) for preparing peptide-MUA/ AuNC. ${ }^{88}$ The synthesized AuNCs displayed two fluorescence emission peaks at 465 and $680 \mathrm{~nm}$. After being adsorbed on the GO surface via electrostatic interaction, the AuNC fluorescence was quenched via FRET. When exposed to MMP-9, the surface peptide substrates were hydrolyzed, and the AuNCs were released from the GO surface, leading to recovery of fluorescence. The restored fluorescence at $640 \mathrm{~nm}$ was used for quantitative analysis of MMP-9, and the detection limit was $2.5 \mathrm{ng} \mathrm{mL}^{-1}$. The peptide-MUA/AuNC/GO nanocomplex was further applied for determination of secreted MMP-9 from MCF-7 cells, and the results were well consistent with the gold standard ELISA assay. Similarly, CNT with long-range nanoscale energy transfer property has also been used as an effective fluorescence quencher. Huang et al. designed a multiwalled CNT (MWCNT)-based multiplex protease (MMP-2, MMP-7 and UPA) biosensor by attaching different dye-labeled peptide substrates on MWCNT. ${ }^{89}$ The involvement of MWCNT could adequately reduce background fluorescence, resulting in high detection sensitivity.

Particularly, GO also emits fluorescence via recombination of electron-hole pairs in the disrupted $\pi$ network at oxidation 

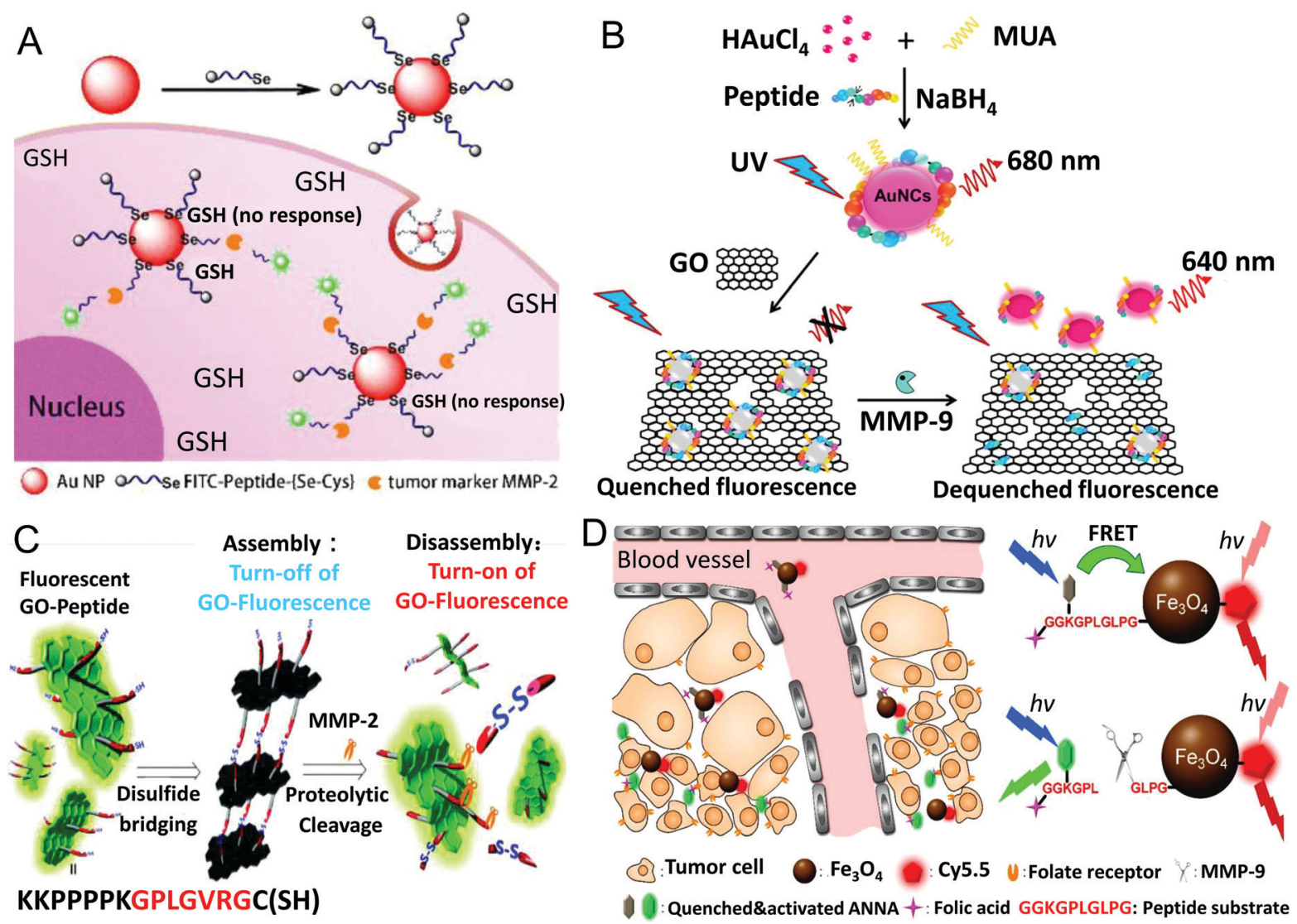

Fig. 5 (A) An Au-Se bond-based AuNP-FITC nanoprobe for sensing MMP-2 in tumor cells with a high level of thiol compounds (reprinted with permission from ref. 82. Copyright 2018, American Chemical Society). (B) Schematic illustration of the preparation of peptide stabilized fluorescent gold nanoclusters (peptide-MUA/AuNC), and the application of the peptide-MUA/AuNC/GO nanocomplex for detecting MMP-9 (reprinted with permission from ref. 88. Copyright 2017, Elsevier). (C) Schematic diagram of the fluorescent GO-based biosensor for turn on detection of MMP-2 via peptidemediated assembly and proteolytic cleavage-triggered disassembly (reprinted with permission from ref. 101. Copyright 2016, Royal Society of Chemistry). (D) Description of the dual-ratiometric fluorescent probe for simultaneously sensing MMP-9 activity and pH in the tumor microenvironment with Fe ${ }_{3} \mathrm{O}_{4}$ NPs as the quencher (reprinted with permission from ref. 91. Copyright 2017, American Chemical Society).

sites, and the emission wavelength can be easily tuned from the visible to the NIR region by varying the extent of oxidation. ${ }^{104}$ In addition, the GO fluorescence shows good resistance to photobleaching and chemical degradation, benefiting quantitative analysis. To exploit GO as a fluorescence donor in FRETbased biosensors, Kim's group covalently attached quencher labeled peptide substrates on fluorescent GO, and employed the complex for sensing MMP-2. ${ }^{100} \mathrm{QXL}_{570}$ was screened as the most effective quencher for GO; the as-prepared GO-peptideQXL biosensor gave a very fast response $\left(0.76 \mu \mathrm{mol} \mathrm{L}^{-1}\right.$ for $K_{\mathrm{m}}$ and $0.49 \mathrm{~min}^{-1}$ for $V_{\max }$ ) to MMP-2 with a detection limit of $2.0 \mathrm{ng} \mathrm{mL}{ }^{-1}$, and showed outstanding stability for long-term storage. In their follow-up work, GO-based quencher-free turn-on fluorescence biosensors were developed for MMP-2 detection, in which GO fluorescence was self-quenched via the peptide-induced assembly. ${ }^{101,102}$ As shown in Fig. 5C, GO was functionalized with MMP-2 peptide substrates bearing thiol groups, then selfassembled via formation of disulfide bonds, resulting in selfquenching of GO fluorescence. ${ }^{101}$ In the presence of MMP-2, the GO assembly was disintegrated by proteolytic cleavage, leading to recovery of GO fluorescence. The detection limit of the GO assembly biosensor for MMP-2 was $2.0 \mathrm{ng} \mathrm{mL} \mathrm{m}^{-1}$, which was comparable to FRET-based biosensors. Lately, they further improved the fluorescence properties of $\mathrm{GO}$ by heteroatomdoping; the obtained N,S-doped GO (NSGO) displayed 10 times higher fluorescence intensity, and was more resistant to the $\mathrm{pH}$ and polarity of the solution. ${ }^{102}$ Benefiting from the enhanced optical properties of NSGO, when applied to detect MMP-2, the NSGO-peptide assembly exhibited much better performance than the pristine GO-peptide assembly, e.g., ten times higher sensitivity and a wider dynamic range.

In addition, PDANPs $,{ }^{90} \mathrm{Fe}_{3} \mathrm{O}_{4} \mathrm{NPs},{ }^{91}$ metal organic frameworks (MOFs) $)^{99}$ and poly $\left(m\right.$-phenylenediamine) (PMPD) ${ }^{106}$ have also been used as quenchers in FRET-based biosensors for MMP detection. Taking advantage of the super fluorescence quenching ability of PDANPs, Xu et al. presented a four-color fluorescent nanoprobe for multiplex detection of four tumorrelated proteases (UPA, MMP-2, MMP-7 and cathepsin B) in living cells by self-assembling four different dye labeled peptide substrates on the PDANP surface. ${ }^{90}$ Due to the good biocompatibility of PDANPs, the nanoprobe was internalized into cells via endocytosis for simultaneously imaging four proteases in living cells. 
Besides magnetic properties, $\mathrm{Fe}_{3} \mathrm{O}_{4}$ NPs display broad featureless absorption in almost the entire visible region, serving as a good candidate for quenching fluorophores with emission in the visible region. For instance, Ma et al. developed an activated dual-ratiometric fluorescent probe for simultaneously sensing MMP-9 activity and $\mathrm{pH}$ in the tumor microenvironment with $\mathrm{Fe}_{3} \mathrm{O}_{4}$ NPs as the quencher. ${ }^{91}$ As shown in Fig. 5D, the probe was designed by conjugating Cy5.5 and a pH-sensitive dye ANNA labeled MMP-9 peptide substrate on $\mathrm{Fe}_{3} \mathrm{O}_{4}$ NPs. The fluorescence of ANNA in the visible region was quenched by the $\mathrm{Fe}_{3} \mathrm{O}_{4}$ NPs, while the Cy5.5 emission in the NIF region remained constant, and served as an excellent internal reference to construct a ratiometric fluorescent probe. In the presence of MMP-9, the peptide was cleaved and the fluorescence of ANNA was recovered. The MMP-9-dependent fluorescence enhancement of ANNA was used for quantitatively determining MMP-9 activity. In addition, the probe was further modified with folic acid (FA) on the free C-terminal of the peptide for tumor targeting. The probe performed well in multimodal imaging of MMP-9 and $\mathrm{pH}$ at tumor sites in vivo.

QDs are popular semiconductor fluorescent nanomaterials, which exhibit unique properties, such as high brightness and remarkable photostability. For instance, the molar extinction coefficients of QDs are 10-100 times higher than those of organic dyes, thus QDs can absorb many more photons to emit brighter fluorescence. The emission of QDs can be tuned from the UV to the infrared (400-1350 nm) by adjusting their sizes. Because of the broad absorption spectra, and narrow and wellresolved emission peaks, various QDs with different emissions could be excited simultaneously by a single light source, allowing multiplex sensing. ${ }^{105}$ In FRET-based biosensors for MMPs, QDs mainly serve as donors, and are connected to the desired acceptors through the link of peptide substrates. ${ }^{76,87,92-94}$ Upon being cleaved by MMPs, the FRET process is terminated along with the recovery of QD fluorescence. Chung et al. presented a multifunctional QD-based FRET biosensor for profiling MT1MMP activity in single cells, which consisted of CdSe/ZnS QDS and a Cy3-labeled bent peptide. ${ }^{92}$ As exhibited in Fig. 6A, the peptide was composed of a QD binding domain $(6 \times$ His $)$, nine positively charged arginines $(9 \times A r g)$, a cell targeting sequence $(3 \times$ RGD), an MT1-MMP specific sequence (AHLR) and eight negatively charged glutamates $(8 \times$ Glu). The peptide was selfassembled on QDs via metal-mediated chelation with $6 \times$ His, and bent into a hairpin loop due to the electrostatic interaction between $9 \times \operatorname{Arg}$ and $8 \times$ Glu, causing the close proximity of Cy3 to the QD surface and occurrence of FRET. After being cleaved by MT1-MMP tethered on the cell surface, peptide fragments with Cy3 and $8 \times$ Glu were released from the QDs, and the disruption of FRET induced the enhancement of the QD emission and decrease of Cy3 fluorescence. Therefore, a ratiometric fluorescence biosensor based on the emission ratio of QDs/Cy3 was developed for quantifying the MT1-MMP activity. Meanwhile, QDs with peptide segments containing $9 \times \operatorname{Arg}$ and $3 \times$ RGD moieties penetrated into cells after cleavage; the intracellular QD signals provided an alternative indicator of MT1-MMP cleavage and integrin receptor expression. Through the dual index readout, the QD-FRET biosensor was capable of distinguishing cell lines with different invasion or adhesion potentials at the single cell level.

Similarly, Wang et al. prepared a FRET-based prodrugtype and MMP-2-responsive nanoprobe for in vivo detection of tumors with QDs as the donor. ${ }^{93}$ Specifically, low-molecularweight protamine (LMWP) modified peptide containing a MMP-2 cleavage sequence and a quencher (QSY21) were

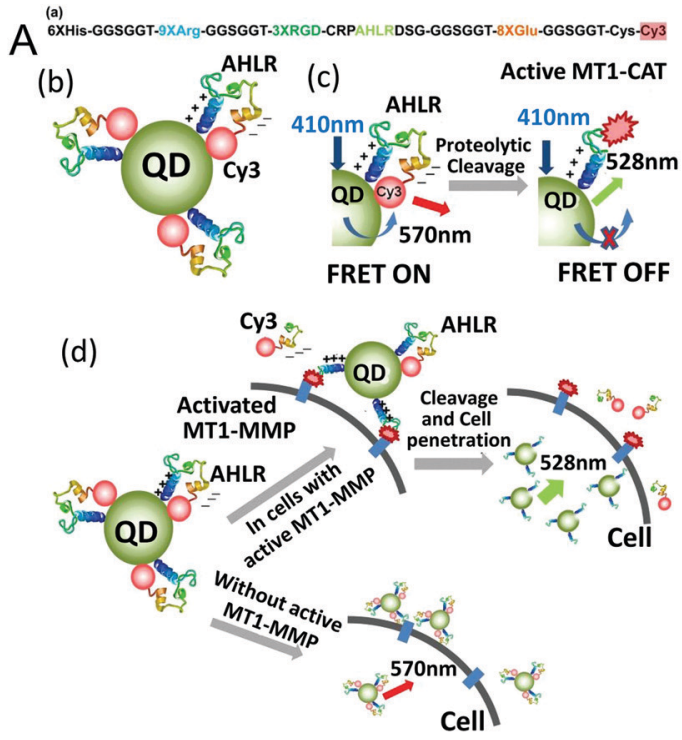

$B$

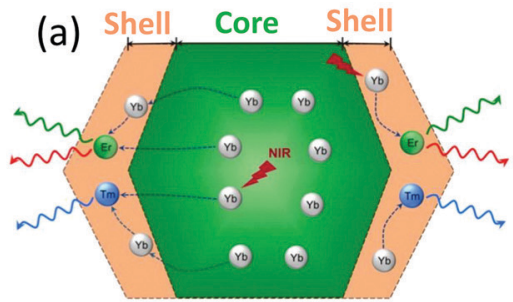

(b)

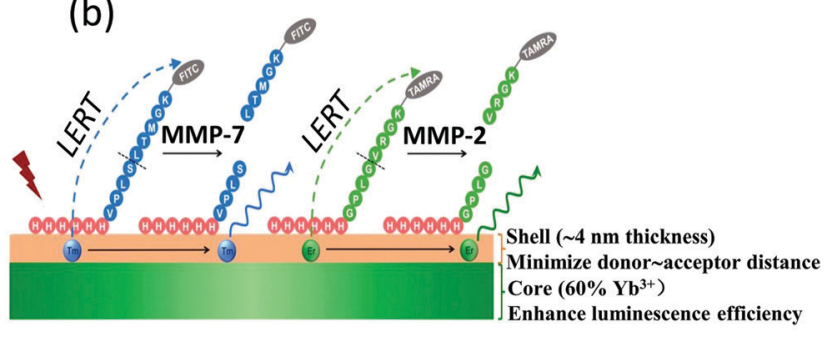

Fig. 6 (A) Illustration of the structure and activation mechanism of the QD-FRET nanosensor, and the application for visualizing MT1-MMP activity and integrin receptor expression in single cells (reprinted with permission from ref. 92. Copyright 2015, American Chemical Society). (B) Scheme of the multi-color UCNPs as a ratiometric sensor for multiplex detection of MMP-2 and MMP-7 (reprinted with permission from ref. 98. Copyright 2018, American Chemical Society). 
self-assembled on low-molecular-weight heparin (LMWH) modified CdTe QDs via the strong affinity between the anionic heparin and cationic protamine. By LMWH modification, the stability of the CdTe QDs was markedly improved, and the toxicity of the QDs was distinctly reduced. The particle size of the nanoprobe $(204 \mathrm{~nm})$ facilitated tumor accumulation via the EPR effect, and the negative charge on the surface reduced nonspecific binding with serum proteins in blood. After responding to MMP-2, QSY21 was away from the QDs, causing the recovery of QD fluorescence. The remaining LMWP on the QD surface acted as a cell-penetrating peptide (CPP) for cellular uptake of the activated probe, allowing determination of the MMP-2 activity in cells. Moreover, the challenging brain tumor detection and imaging were achieved by this nanoprobe via incorporating a brain-targeting $\mathrm{T} 7$ sequence into LMWP.

As a kind of lanthanide doped inorganic crystals with antiStokes luminescence, UCNPs are also employed as donors to construct FRET-based biosensors. Compared with traditional luminescence materials with Stokes shifts, UCNPs with emission in the visible region are excited by NIR light, which effectively ameliorates the interference of autofluorescence and scattered light from biomolecules, resulting in high sensitivity and good specificity. Liu's group proposed a FRET-based biosensor for ultrasensitive detection of MMP-2 with UCNPs as the donor, and carbon nanoparticles (CNPs) as the acceptor. ${ }^{95}$ Due to $\mathrm{sp}^{2}$ orbital hybridization, the $\pi$-rich electronic structure of CNPs endows them with strong fluorescence quenching ability, and enables easy conjugation of biomolecules containing aromatic motifs via $\pi-\pi$ stacking interactions. Therefore, CNPs and PEI coated UCNPs were linked by a peptide chain containing an MMP-2 cleavage sequence and a $\pi$-rich motif $\left(\mathrm{NH}_{2}\right.$-GHHYY GPLGVRGC-COOH), leading to quenched UCNP fluorescence. In the presence of MMP-2, the peptide was cleaved into two parts, and the UCNPs were separated from the CNPs, accompanied by recovery of UCNP fluorescence. Under the optimal conditions, the fluorescence recovery was in proportion to the MMP-2 concentration in the range of $10-500 \mathrm{pg} \mathrm{mL}^{-1}$ with a detection limit of $10 \mathrm{pg} \mathrm{mL}^{-1}$. Since NIR excitation of UCNPs could effectively eliminate the background interference, the biosensor was well applied for MMP-2 detection in human plasma and whole blood samples. In their subsequent work, the UCNP-TAMRA FRET assay was integrated with a paperbased microfluidic device for sensitive detection of MMP-2. ${ }^{96}$ In Chan's work, UCNPs were coated with a $\mathrm{SiO}_{2}$ shell to improve the biocompatibility, and then coupled with $3.5 \mathrm{~nm}$ AuNPs via polypeptides to construct a FRET-based MMP-2 biosensor. ${ }^{97}$ The UCNP@p-Au nanoprobe showed an extremely low detection limit of $4 \times 10^{-4} \mathrm{pg} \mathrm{mL}^{-1}$ for MMP-2. Additionally, after being cleaved by MMP-2, the modified FA on the UCNPs facilitated the cellular uptake of the UCNPs, allowing sensing MMP-2 in head and neck cancer cells. Taking advantage of the dopant-dependent multicolor emission of UCNPs, Cao et al. developed a ratiometric biosensor for multiplex determination of MMP-2 and MMP-7 activities. ${ }^{98}$ As shown in Fig. 6B, a NaYF 4 : $\mathrm{Gd}^{3+} / \mathrm{Yb}^{3+} @ \mathrm{NaYF}_{4}: \mathrm{Yb}^{3+} / \mathrm{Tm}^{3+} / \mathrm{Er}^{3+}$ core-shell UCNP structure was prepared, which displayed multicolor emission (blue, green, red). MMP-7 and MMP-2 peptide substrates labeled with FITC and TAMRA were self-assembled on the surface of UCNPs via polyhistidine tails. Because of the luminescence resonance energy transfer (LRET) from dopants in the shell to fluorophores on the surface, the blue emission of $\mathrm{Tm}^{3+}$ was quenched by FITC, and the green emission of $\mathrm{Er}^{3+}$ was quenched by TAMRA, while the red emission of $\mathrm{Er}^{3+}$ remained unchanged, and was used as an internal reference for ratiometric sensing. Once treated with the target MMP, the peptide was specifically cleaved, and the corresponding emission of the UCNPs was recovered. Because of the sharp emission bandwidths, MMP-2 and MMP-7 could be specifically discriminated with little crossreactivity. The detection limits of the multiplex assay for MMP-2 and MMP-7 are $2.2 \mathrm{ng} \mathrm{mL} \mathrm{m}^{-1}$ and $13.9 \mathrm{ng} \mathrm{mL}^{-1}$.

(3) Nanomaterials as BRET acceptors. Bioluminescent probes have also been used as energy donors in the resonance energy transfer process, which is called BRET. Unlike FRET, no external light excitation is required in BRET, but an enzyme (such as luciferase) is essential, which emits light during the catalytic oxidation of its substrates. When the emission spectrum of bioluminescence is overlapped with the excitation spectrum of another fluorophore, nonradiative energy transfer from the bioluminescent donor to the appropriate acceptor occurs. ${ }^{107}$ It has been reported that a BRET system is more sensitive than FRET for analysing protease hydrolysis. Rao's group has developed several BRET-based sensing systems for determination of MMP-2 activity. ${ }^{108-111}$ Kim et al. proposed a bioluminescent nanosensor for MMP-2 detection using Renilla luciferase (Luc8)-AuNP conjugates. ${ }^{108}$ Luc8 was expressed with an MMP-2 substrate peptide (IPVSLRSG) and fused with Mex GyrA intein protein, and then conjugated to AuNPs in a site-specific orientation via click chemistry and an inteinmediated ligation method. It was demonstrated that the bioluminescence of Luc8 could be effectively quenched by AuNPs, and then recovered after the linking peptide was specifically cleaved by MMP-2. Other than AuNPs, QDs also served as acceptors of bioluminescence. ${ }^{109,110}$ In Yao's work, Luc8 fused with an MMP-2 substrate peptide and a six-histidine tag $(6 \times \mathrm{His})$ was conjugated to carboxylated QDs via $\mathrm{Ni}^{2+}$-mediated coordination-chelation interactions. ${ }^{109}$ The bioluminescent emission of Luc8 at $480 \mathrm{~nm}$ was quenched by the QDs, while the emission of the QDs at $655 \mathrm{~nm}$ was increased. Cleavage of the peptide by MMP-2 disrupted the BRET process, resulting in recovered bioluminescence and decreased QD fluorescence. Instead of measuring a single bioluminescence intensity, the ratio of two emissions (BRET ratio) was used for quantitative analysis of MMP-2 activity, improving the accuracy of the sensing system. The multiplex protease sensing capability of the QD-BRET system was further explored by Xia et al.; they developed three nanosensors for MMP-2, MMP-7 and urokinase-type plasminogen activator (UPA) detection by fusing Luc8 with the corresponding substrate peptides. ${ }^{110}$ Compared with FRET, BRET offers several advantages, such as no photobleaching of fluorophores, and no interference from autofluorescence. But the activity of luciferase is susceptible; 
its stability should be improved for long-term and in vivo monitoring of MMP activities.

FRET-based biosensors depend on the energy transfer between fluorophores and quenchers, but the double labeling of peptide substrates is complicated, and may affect the interaction with MMPs. To circumvent this issue, Su et al. proposed a luminescent biosensor for MMP-9 detection with an $\operatorname{Ir}($ III) solvent complex as the signal transducer and $\mathrm{Fe}_{3} \mathrm{O}_{4}$ NPs as a peptide substrate carrier. ${ }^{112}$ Interestingly, the $\operatorname{Ir}(\mathrm{III})$ solvent complex exhibited very weak emission in aqueous solution, but the luminescence would be significantly enhanced after specific recognition of His. Peptides containing MMP-9 cleavage sequences and His-rich motifs were attached on $\mathrm{Fe}_{3} \mathrm{O}_{4}$ NPs. When treated with MMP-9, the peptide fragments with His-rich motifs were cleaved off, and released into solution. After magnetic separation, the Ir(III) solvent complex was added into the supernatant, and selectively reacted with His and produced strong emission. The readily fabricated "signal-on" luminescent biosensor showed a detection limit of $2.1 \mathrm{pmol} \mathrm{L}^{-1}$, and was capable of determining MMP-9 in MCF-7 cell lysates.

3.2.1.3 SPR-based biosensors. As described in the immunoassays, SPR is a prevailing label-free method for monitoring the reaction process on the sensing surface. Degradation of the immobilized receptor would also cause a change in RI of the sensing layer. Therefore, the proteolytic activity of MMPs could also be determined by SPR-based biosensors via hydrolysis of immobilized substrates. ${ }^{113-115}$ Jung et al. proposed an array-based SPR biosensor for high-throughput analysis of
MMP-3 activity by immobilizing gelatin on amine-modified gold arrays. ${ }^{113}$ Gelatin proteolysis by MMP-3 led to a blue shift of the SPR wavelength, which could be monitored via the linescanning mode of the spectral SPR sensor. Thus, the MMP-3 activity was displayed as color spectra from blue to red. In the work of Hong et al., PEGylated gold nanorods (PGNRs) were assembled on glass slides as an LSPR substrate, and functionalized with specific peptide substrates for sensing MMP-14 of invasive cancer cells. ${ }^{114}$ The sensitivity of the as-prepared LSPR substrate to the change of RI was $169.8 \mathrm{~nm}$ per RI unit, enabling one to detect the target at low concentration. Recently, in order to reduce the nonspecific binding in real sample detection, Park et al. reported an SPR biochip with a PEGgrafted antifouling surface for label-free and real-time determination of MMP-7 activity in cell culture media. ${ }^{115}$ Via plasma copolymerization (PCP) of PEG and ethylenediamine (EDA) precursors, a PcP-PEG-EDA (PcP-PE) film was deposited on a gold sensor chip for SPR measurement. The amine density in the polymer film could be well controlled to achieve high binding capacity for biomolecules and low nonspecific adsorption. As shown in Fig. 7A, the PcP-PE film-grafted chip surface was firstly functionalized with biotin, and then bound with streptavidin (SA) for specific immobilization of the probe. Renilla luciferase mutant protein (Rluc8) fused with an MMP7-specific peptide substrate (m7pep) was tagged with biotin, and the resulting Rluc8-m7pep-biotin conjugate was immobilized on the chip surface, serving as a protein probe for sensing MMP-7. Once treated with MMP-7, the peptide was cleaved, and Rluc8 was released from the chip surface, causing a decrease in
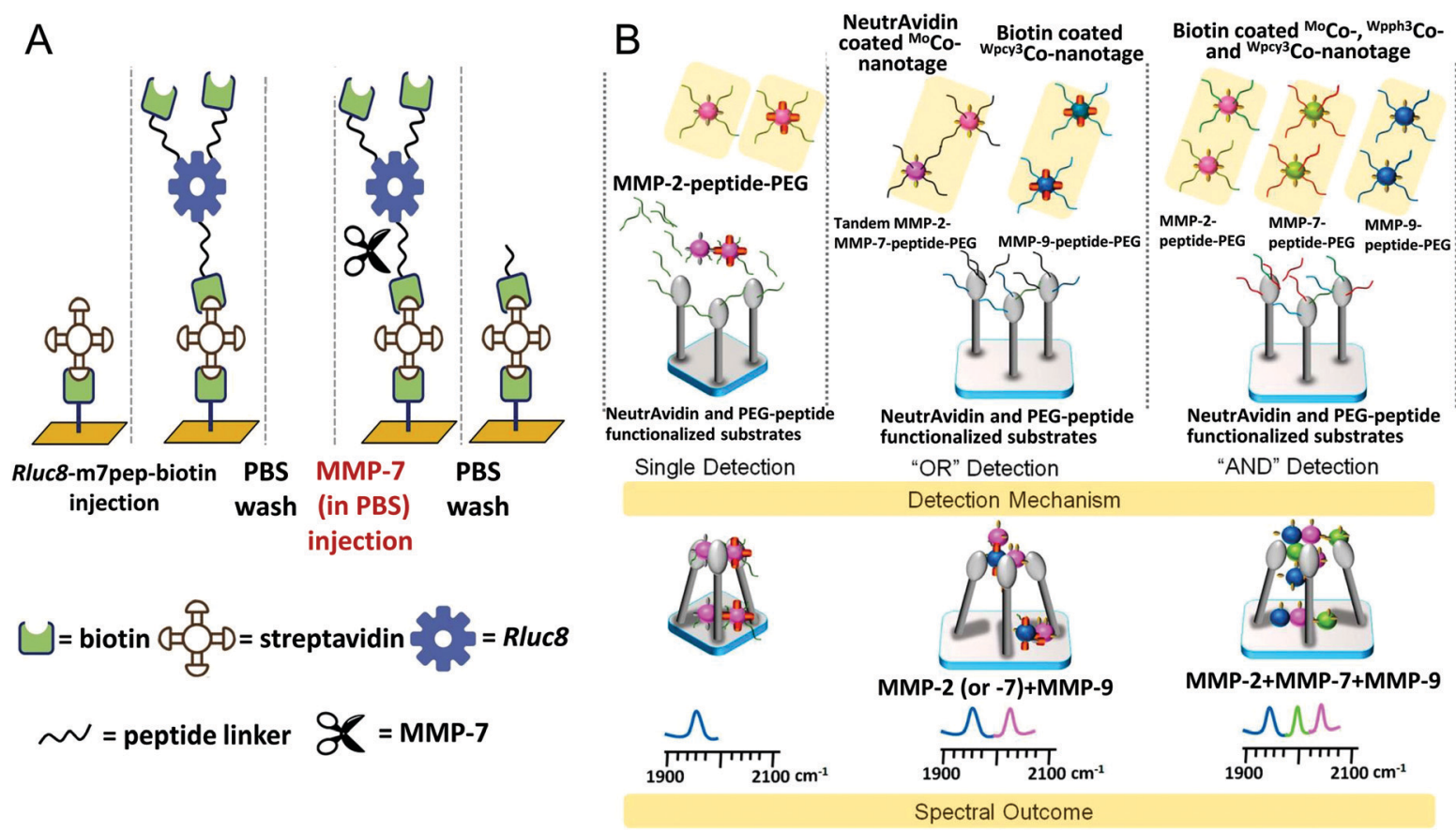

Fig. 7 (A) Description of the SA-conjugated SPR chip for real-time monitoring of MMP-7 activity in cell culture medium with a PCP-PEG-EDA (PCP-PE) film and biotinylated luciferase probes (reprinted with permission from ref. 115. Copyright 2019, Elsevier). (B) Cleave-and-bind mechanism-based SERS biosensor for logical multiplex analysis of MMPs using a gold-coated nanopillar substrate and optical interference-free CO-nanotags (reprinted with permission from ref. 117. Copyright 2017, American Chemical Society). 
the SPR sensorgram. Due to the outstanding performance of the PcP-PE surface in specific binding, surface passivation was no longer needed in the SPR assay. The proposed PcP-PE-based SPR biosensor allowed label-free detection of MMP-7 activity in complex samples with high sensitivity and fewer nonspecific events. In addition, as a bioluminescence reporter, the MMP-7 cleavage-induced Rluc8 dissociation could also be monitored by bioluminescence imaging.

3.2.1.4 SERS-based biosensors. Due to the intrinsic advantages, SERS has also been exploited for determination of MMP activities, especially for multiplex detection. ${ }^{116-118}$ SERS-based biosensors are typically composed of metal substrates, MMP cleavage peptide linkers and Raman reporters. Gong et al. developed a cleave-and-bind mechanism-based SERS platform for multiplex detection of MMP-2 and MMP-7 activities. ${ }^{116}$ The platform was mainly composed of a bimetallic-film-overnanosphere (BMFON) substrate and AuNPs. The BMFON substrate was prepared by depositing polystyrene nanospheres on glass slides, followed by sputtering an $\mathrm{Ag}$ underlayer and $\mathrm{Au}$ overlayer. The as-prepared substrate not only retained the strong enhancing property of $\mathrm{Ag}$, but also protected $\mathrm{Ag}$ from oxidation by the $\mathrm{Au}$ coating. Moreover, the outer $\mathrm{Au}$ film facilitated the conjugation of biomolecules via Au-thiol chemistry. Biotin-NeutrAvidin conjugates along with MMP-2 and MMP-7 peptide substrates were attached on the BMFON substrate for sensing. Meanwhile, AuNPs were also modified with specific MMP peptide substrates, and further loaded with a Raman reporter and biotin. Initially, the binding of AuNPs with the BMFON substrate was hindered by MMP peptide chains on the surface of the two components. After being cleaved by the corresponding MMP, the binding sites were available, and AuNPs were anchored on the BMFON substrate, leading to enhanced Raman signals. The Raman peaks of 4-aminothiophenol (ATP) at $1584 \mathrm{~cm}^{-1}$ and 2-naphthalenethiol (NT) at $1377 \mathrm{~cm}^{-1}$ were selected for specific detection of MMP-2 and MMP-7, respectively. By measuring the corresponding SERS peak intensity, the individual MMP in the range of $1 \mathrm{ng} \mathrm{mL}^{-1}$ to $40 \mu \mathrm{g} \mathrm{mL} \mathrm{m}^{-1}$ could be determined. In their subsequent work, Gong et al. proposed a new class of optical interference-free SERS nanotags (metal carbonyl-based nanotags), and employed them to establish a SERS-based biosensor for multiplex detection of MMPs (MMP-2, -7 and -9) without any cross-talk. ${ }^{117}$ The CO exhibited strong stretching vibrations in the mid-IR region (1800-2200 $\mathrm{cm}^{-1}$ ), which could effectively avoid the interference from absorbance of biomolecules. As shown in Fig. 7B, they presented a gold-coated nanopillar substrate, which was demonstrated to be capable of significantly enhancing the SERS intensity of aggregated CO-nanotags. Three CO-nanotags $\left({ }^{\mathrm{Mo}} \mathrm{CO},{ }^{\mathrm{Wpph} 3} \mathrm{CO}\right.$ and ${ }^{\mathrm{Wpcy} 3} \mathrm{CO}$ ) were selected, and conjugated with MMP-2, MMP-7 and MMP-9 peptide substrates for cleavage, respectively. Meanwhile, they were also conjugated with biotin or NeutrAvidin for aggregating on the nanopillar substrate. Based on the previously described cleave-and-bind mechanism, the MMP activity was indicated by the Raman intensity of the target $\mathrm{CO}$ peak. Combined with Boolean logic, the biosensor was exploited for logical multiplex detection of MMPs. Benefiting from the optical interference-free nanotags, in the logical "AND" system, multiplex MMPs were detected without the use of an internal standard, and the detection limits were $0.05 \mathrm{ng} \mathrm{mL}{ }^{-1}$ for MMP-2, $0.05 \mathrm{ng} \mathrm{mL} \mathrm{m}^{-1}$ for MMP-7 and $0.1 \mathrm{ng} \mathrm{mL} \mathrm{m}^{-1}$ for MMP-9. The clinical practicality of the biosensor was validated by detecting MMPs in brain blood of rats with acute focal cerebral ischemia. Instead of measuring the enhanced Raman intensity, Lin et al. proposed a GO-based SERS biosensor for MMP-2 by monitoring the spectral shift. ${ }^{118}$ When ruthenium carbonyl (Ru-CO) clusters were adsorbed on monolayer graphene (MG), ruthenium oxide nanoparticles $\left(\mathrm{RuO}_{2} \mathrm{NPs}\right)$ formed instantaneously, which could cause a spectral shift of the characteristic G band of MG. TAMRA-labeled PEGylated MMP-2 peptide substrates were self-assembled on MG via $\pi-\pi$ interactions, precluding the adsorption of Ru-CO. Upon exposure to MMP-2, the peptide was cleaved away from the MG surface, and the following incubation with Ru-CO led to the formation of $\mathrm{RuO}_{2}$ NPs. The MMP-triggered doping of $\mathrm{RuO}_{2}$ NPs caused a blue shift of the $\mathrm{G}$ band peak for MG, which was linear with the MMP-2 concentration. The cost effective and simple biosensor displayed a detection limit of $17 \mathrm{ng} \mathrm{mL}{ }^{-1}$, and the feasibility was verified by detecting MMP-2 in simulated clinical serum samples, showing great potential for clinical biomarker detection.

3.2.1.5 Porous silicon-based optical biosensors. Porous silicon (pSi) is a promising optical transducer, and can be easily prepared by electrochemical etching of pure single crystalline silicon, producing a nanostructure with pores perpendicular to the surface. The porosity and pore size of pSi can be tuned by adjusting the synthesis conditions to create photonic crystals which reflect light at well-defined wavelengths. When recognition molecules are attached onto the pore wall of $\mathrm{pSi}$, the average RI of the pSi structure is changed due to the replacement of water with lower RI. The position of the reflectivity peak of pSi is sensitive to the change of RI, which can be monitored by the optical reflectivity spectrum. ${ }^{119}$ The excellent optical reflectivity properties of pSi make it a good candidate for constructing label-free biosensors. In the past decade, pSi-based biosensors have been applied for determining MMP activities. ${ }^{120-126}$ Gao et al. proposed a label-free colorimetric biosensor for detecting MMP-2 by simply spin-coating a gelatin gel layer on a pSi film. ${ }^{120}$ When MMP-2 containing samples were spotted, the digested products of gelatin entered the pSi pores, causing a color change. Owing to the sensitive color change, the biosensor was convenient for observation by the naked eye, and allowed detecting as low as $0.1 \mathrm{pg}$ MMP-2 in a $1 \mu \mathrm{L}$ sample. Gooding's group improved the sensitivity by firstly modifying the pore wall with antifouling $\mathrm{EG}_{6}$ species, and then coupling with gelatin for protease digestion. ${ }^{121}$ By monitoring the optical response, the proteolytic activity of MMP-9 can be determined down to 1-2 pmol $\mathrm{L}^{-1}$ within $1 \mathrm{~h}$. In their subsequent work, they proposed a generic strategy to construct sensitive pSibased MMP biosensors. The pSi pore wall was firstly modified with a layer of antifouling copolymer via click chemistry, then coupled with MMP specific peptide substrates, and finally attached to a sacrificial polymer. ${ }^{122,123}$ After being exposed to MMPs, the polymer network was digested, and the peptide 
fragments carrying sacrificial polymer left the pores, resulting in a blue shift of the reflectivity peak of pSi (as shown in Fig. 8A). The filling of the enzymatically degradable polymerpeptide network improved the optical response of the bio sensor, leading to enhanced sensitivity. The biosensor performed well in a quantifying picogram of MMP-2 and MMP-9 secreted by primary retinal pigment epithelial (RPE) and iris pigment epithelial (IPE) cells. ${ }^{123}$

In addition to being sensitive to RI changes, pSi shows fluorescence enhancement for specific fluorophores embedded in the matrix. Taking advantage of this feature, several FRETbased biosensors have been designed for determining MMP activity. Krismastuti et al. functionalized a porous silicon resonant microcavity (pSiRM) with fluorogenic MMP peptide substrates containing an EDANS/Dabcyl pair, and employed the hybrid for sensitive detection of MMP-1 in wound exudate. ${ }^{124}$ The immobilized peptide substrates were cleaved by MMP-1, leaving the fragments with EDANS on the matrix of PSiRM. The recovered fluorescence of EDANS was further enhanced by pSiRM, resulting in improved sensitivity of the optical biosensor with an ultra-low detection limit of 0.75 amol $\mathrm{L}^{-1}$. Moreover, determination of MMP in wound fluid was accomplished in $15 \mathrm{~min}$, demonstrating the potential for POC application. Later, they prepared a peptide array on pSiRM, and constructed a FRET-based biosensor for multiplex detection of Sortase A (SrtA) and MMP-1 in bacterial culture medium and wound fluid. ${ }^{125}$ In their follow-up work, they integrated the pSiRM-based FRET biosensor with a magnetic nanoparticle (magNP)-based immunocapture assay for type-selective detection of MMPs. ${ }^{126}$ As shown in Fig. 8B, MMP antibody modified magNPs were used for specific capture of target MMPs in samples, and were separated by a magnet to eliminate interferences. When released into the FRET peptide functionalized pSiRM, the active MMPs bound on the magNPs hydrolyzed the immobilized peptide substrates, inducing the recovery and enhancement of EDANS fluorescence. MMP-1 and MMP-9 in human chronic wound fluid samples were successfully detected and distinguished with the biosensor, showing the great potential for POC diagnosis.
3.2.2 Electrochemical biosensors. In addition to immunoreactions between MMPs and corresponding antibodies, proteolysis between MMPs and corresponding substrates could also be detected by electrochemical biosensors. Electrochemical transducers convert the MMP-triggered hydrolysis of substrates into measurable electrochemical signals. A variety of electrochemical/electrical techniques have been exploited to design biosensors for determining active MMPs, such as voltammetry (cyclic voltammetry (CV), ${ }^{127,128}$ SWV ${ }^{128-131} \mathrm{DPV},{ }^{132-136}$ stripping voltammetry ${ }^{137}$ ), EIS, ${ }^{138,139}$ electrochemiluminescence (ECL) $)^{140-142}$ and field-effect transistors (FET). ${ }^{143-146}$ Among these electrochemical biosensors, EIS directly measures the change in impedance of the electrode surface, allowing monitoring the cleavage events of MMPs in a label-free manner. Whereas, other biosensors measure the changes in electrochemical signal of electroactive species on the electrode surface, which are dependent on the concentration of active MMPs.

Typically, electrochemical MMP biosensors follow the turn-off strategy; peptide substrates labeled with electroactive tags are immobilized on the electrode surface, after being cleaved by MMPs, the peptide fragments with electroactive reporters are released from the electrode surface, resulting in decreased electrochemical signal. ${ }^{127-130}$ For instance, Lee et al. presented a concentric two-electrode system-based MMP-9 biosensor by measuring the decreased electrical tunneling current. ${ }^{127}$ Similarly, Shin et al. immobilized MB-labeled peptide substrates on an Au electrode surface for sensing cell-secreted MMP-9 activity by SWV measurement. ${ }^{129}$ PEG gel was micropatterned around the Au electrode arrays on a glass slide, and further functionalized with CD14 antibodies to define annular regions for cell adhesion. Integrating with a microfluidics device for cell capture and activation, the MMP-9 activity from activated U-937 cells could be monitored up to $4 \mathrm{~h}$, and the determined release rate was $0.65 \mathrm{pg}$ cell $^{-1} \mathrm{~h}^{-1}$. To achieve better performance, Zheng et al. introduced a dual-reaction triggered signal amplification strategy into an amperometric biosensor for ultrasensitive detection of MMP-7. ${ }^{130}$ As shown in Fig. 9A, the GCE was first modified with MB-sodium alginate hydrogel (MB-SA), and further functionalized with Au-rGO to improve
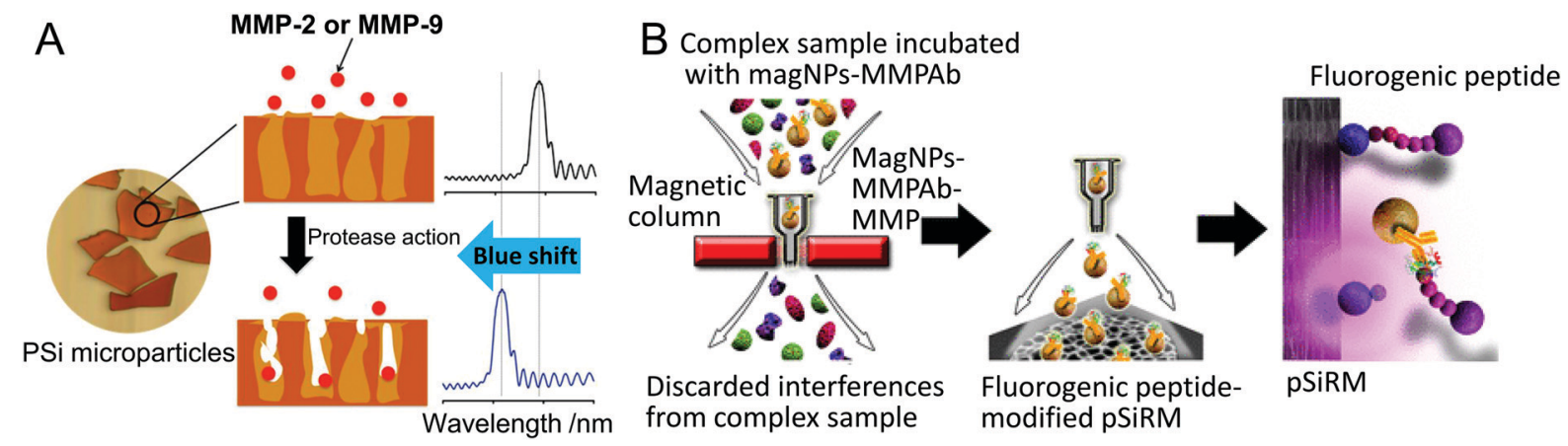

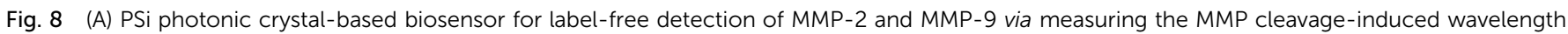

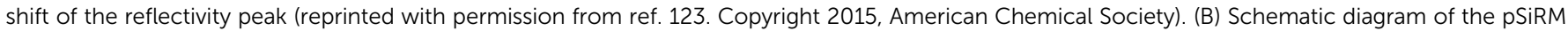

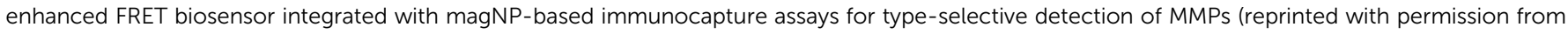
ref. 126. Copyright 2017, American Chemical Society). 

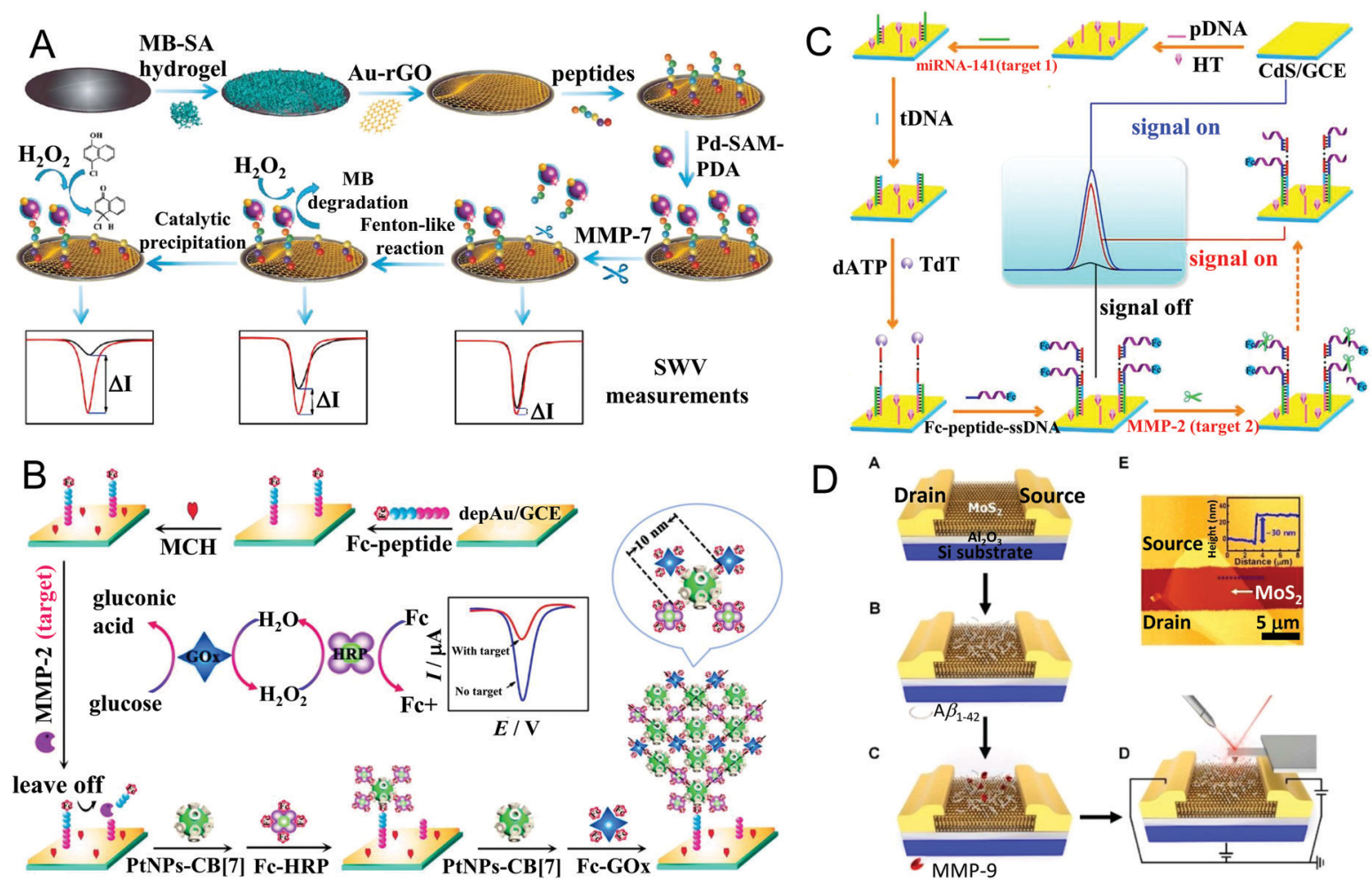

Fig. 9 (A) Schematic illustration of the electrochemical biosensor with a dual-reaction triggered signal amplification strategy for ultrasensitive detection of MMP-7 (reprinted with permission from ref. 130. Copyright 2018, Elsevier). (B) Enzyme cascade amplification-based ultrasensitive electrochemical biosensor for MMP-2 detection with PtNPs as scaffolds to regulate the interenzyme distance (reprinted with permission from ref. 136. Copyright 2017, American Chemical Society). (C) CdS QD-based "on-off-on" ECL biosensor for multiple detection of miRNA-141 and MMP-2, which involved TdT-mediated extension and Fc-induced ECL quenching (reprinted with permission from ref. 142. Copyright 2017, American Chemical Society). (D) Illustration of the $\mathrm{MoS}_{2}$-based FET biosensor for detection of MMP-9 by functionalizing the surface with $A \beta_{1-42}$ as a protein probe and amplifier (reprinted with permission from ref. 146. Copyright 2019, American Chemical Society).

the conductivity of the electrode and facilitate the attachment of MMP-7 specific peptide substrates. The prepared BSA-PdSAM-PDA nanocomposites were coupled to the immobilized peptide, and served as catalytic probes. After the peptide was cleaved by MMP-7, no apparent decrease of peak current $(\Delta I)$ was observed in SWV measurements. Whereas, the remaining BSA-Pd-SAM-PDA nanocomposites triggered dual catalytic reactions, significantly amplifying $\Delta I$ : firstly, the $M B$ in the hydrogel was degraded via a Fenton-like reaction; secondly, 4-CN was catalytically oxidated, producing precipitation with poor electrical conductivity. Benefiting from the dual signal amplification, MMP-7 could be sensitively detected down to $0.1 \mathrm{ng} \mathrm{mL} \mathrm{m}^{-1}$. Instead of a conventional redox indicator, Zheng et al. employed gold-quantum dot (Au-QD) core-satellite nanoprobes as electroactive reporters for dual-channel analysis of MMP-2 based on both anodic stripping voltammetry (ASV) and fluorescence measurements. ${ }^{137}$ MMP-2 specific peptide substrates were attached on an $\mathrm{Au}$ film with free biotin on the $\mathrm{N}$-terminal. After being incubated with MMP-2, SA-functionalized $\mathrm{Au}-\mathrm{QD}$ core-satellite nanoprobes were coupled to the uncleaved peptides via the high affinity with biotin. By dissolving the captured QDs with $\mathrm{HNO}_{3}$, sharp and well-resolved ASV signals of $\mathrm{Cd}$ were generated, and were dependent on the MMP-2 concentration. In addition, specific binding with the dissolved
$\mathrm{Cd}^{2+}$ would significantly enhance the quantum yields of some metal-sensitive dyes, such as Fluo-4 and Rhod-5N. Thus, the enhanced fluorescence intensity could be used to quantify the $\mathrm{Cd}^{2+}$ concentration, which was also the indicator of the MMP-2 concentration. The Au-QD core-satellite nanoprobes prepared by assembling multiple $\mathrm{CdSe}_{0.5} \mathrm{Te}_{0.5}$ QDs on AuNPs served as multiple labels for signal amplification in both ASV and fluorescence measurements. Consequently, MMP- 2 could be detected as low as $0.63 \mathrm{pg} \mathrm{mL} \mathrm{m}^{-1}$ and $0.72 \mathrm{pg} \mathrm{mL}^{-1}$ by the electrochemical and fluorescence channel, respectively. Moreover, the high sensitivity of the biosensor significantly reduced the consumption of clinical sample to $1 \mu \mathrm{L}$, showing great promise for POC testing.

However, the electrochemical biosensors in 'signal-off' mode suffer from limited signal output, inevitably affecting the sensitivity. To address this problem, Yuan's group integrated various signal amplification strategies with electrochemical MMP biosensors, such as a DNA-based amplification strategy ${ }^{131-134}$ and nanomaterial-based amplification. ${ }^{135,136}$ For example, Wang et al. converted MMP-2 analysis into DNA detection, and combined with exonuclease III (Exo III)-assisted cycling signal amplification. ${ }^{133}$ To be specific, magnetic polystyrene microspheres (PSC) and $\mathrm{DNA}_{1}$-modified AuNPs (AuNP$\mathrm{DNA}_{1}$ ) were linked by MMP-2 peptide substrates. The prepared 
PSC-peptide-AuNP-DNA 1 complex was incubated with MMP-2, and separated by a magnet. The released AuNP-DNA $A_{1}$ in the supernate hybridized with MB-labeled complementary $\mathrm{DNA}_{2}$ (MB-DNA ${ }_{2}$ ), forming duplex DNA with a recessed 3 '-terminus on $\mathrm{DNA}_{2}$. Addition of Exo III caused the digestion of $\mathrm{MB}^{-\mathrm{DNA}_{2}}$ in the duplex DNA, and triggered the cycle of DNA hybridization and digestion. The released free $\mathrm{MB}$ molecules were captured by cucurbit[7]uril (CB[7]) on the electrode surface via host-guest interaction, generating a detectable electrochemical signal. The electrochemical responses measured by DPV were proportional to the logarithm of the MMP-2 concentration in the range of $0.5 \mathrm{pg} \mathrm{mL}{ }^{-1}-50 \mathrm{ng} \mathrm{mL}{ }^{-1}$ with a detection limit of $0.15 \mathrm{pg} \mathrm{mL}^{-1}$. In their follow-up work, based on DNA exponential amplification reaction (EXPAR), Wang et al. designed an original two-stage DNA template for highly efficient DNA amplification. With this template, the cleaved DNA with amino acid residues was converted into abundant new amino acid-free DNA, which could be detected by SWV measurement with the assistance of catalyzed hairpin assembly (CHA) recycling. ${ }^{131}$ Therefore, the determination of MMP-7 proteolytic activity was transformed into detection of DNA. Benefiting from the cascade signal amplification of EXPAR and CHA, MMP-7 was detected as low as $0.02 \mathrm{pg} \mathrm{mL}{ }^{-1}$. In addition to the conversion for DNA detection, Kou et al. constructed a DNA enzyme-decorated DNA nanoladder as a signal enhancer for sensitive detection of MMP-7. ${ }^{134}$ Peptide and DNA $\mathrm{S}_{1}$ modified PtNPs $\left(\mathrm{P}_{1}\right.$-PtNP- $\left.\mathrm{S}_{1}\right)$ were immobilized on the electrode surface; after being incubated with MMP-7, the cleaved PtNP-S 1 moieties were detached from the electrode, while the remaining $S_{1}$ on the electrode hybridized with a pair of single stranded DNA $\left(\mathrm{I}_{1}\right.$ and $\left.\mathrm{I}_{2}\right)$ to form single-duplex helixes, and then triggered two hybridization chain reactions (HCR), generating the DNA nanoladder. Meanwhile, a great deal of manganese porphyrin (MnPP) was incorporated in the DNA nanoladder as a catalyst. Owing to the peroxidase-like activity of MnPP, 4-CN was electrocatalyzed to produce insoluble precipitation on the electrode surface, causing a decreased electrochemical signal. By DPV measurement, the peak current was proportional to the MMP-7 concentration in the range of $0.2 \mathrm{pg} \mathrm{mL} \mathrm{m}^{-1}$ to $20 \mathrm{ng} \mathrm{mL} \mathrm{m}^{-1}$, and the detection limit was as low as $0.05 \mathrm{pg} \mathrm{mL}^{-1}$. Later, nanomaterials loaded with electroactive species were also employed for signal amplification. ${ }^{135,136}$ For instance, Kou et al. proposed an electrochemical biosensor with a PtNP-based enzyme cascade amplification strategy for ultrasensitive detection of MMP-2. ${ }^{136}$ As shown in Fig. 9B, Fc labeled peptides were immobilized on the $\mathrm{Au}$ electrode surface, and cleaved by MMP-2. The residual Fc molecules bound with $\mathrm{CB}$ [7] modified PtNPs, and were further loaded with Fc labeled HRP and glucose oxidase (GOx) via host-guest interaction, forming a stable 3D netlike structure carrying plenty of enzymes. Herein, PtNPs with tunable particle size not only served as a scaffold to regulate the interenzyme distance for improving the enzyme catalytic efficiency, but also acted as catalyst to accelerate enzyme cascade amplification. In the presence of glucose, the electrochemical response was significantly enhanced, and the enzyme cascade reaction regulated by $10 \mathrm{~nm}$ PtNPs exhibited the optimal amplification effect. Under the optimal conditions, the DPV response was linear with the logarithmic concentration of MMP-2 in the range from $0.1 \mathrm{pg} \mathrm{mL} \mathrm{m}^{-1}$ to $20 \mathrm{ng} \mathrm{mL}{ }^{-1}$, and the detection limit was $0.03 \mathrm{pg} \mathrm{mL}^{-1}$.

Electrochemiluminescence (ECL) is a powerful tool combining electrochemistry with chemiluminescence. The electrogenerated species on the electrodes undergo highly-energetic electron transfer reactions, and emit light from excited states. Due to its simplicity and high sensitivity, ECL has been widely used in bioanalysis, clinical diagnosis and environmental monitoring. Zhang's group constructed an ECL-based MMP-2 biosensor by self-assembling ECL-emitting species Ru1 labeled peptide (Ru1-peptide) on the surface of an Au electrode. ${ }^{140}$ After being treated with MMP-2, the Ru1-peptide was specifically cleaved and released from the electrode surface, resulting in decreased ECL intensity of Ru1. The detection process was completed within $1 \mathrm{~h}$ with a detection limit of $0.7 \mathrm{ng} \mathrm{mL} \mathrm{m}^{-1}$. By employing two different ECL labels (Ru1 and Ir1) with well-resolved ECL emissions, the assay was further applied for simultaneous detection of MMP-2 and MMP-7. ${ }^{141}$ Instead of using multiplex ECL labels, Nie et al. employed CdS QDs as ECL emitters, and proposed an ECL biosensor for multiple detection of microRNA-141 (miRNA-141) and MMP-2. ${ }^{142}$ As displayed in Fig. 9C, the probe DNA was firstly immobilized on the CdS QD-modified electrode surface, and hybridized with miRNA-141. Then the trigger DNA (tDNA) was captured by miRNA-141, and produced a long ssDNA nanotail via TdT-mediated DNA polymerization. Subsequently, plentiful Fc-peptide-ssDNA complexes with an MMP-2 cleavage sequence were captured by the long ssDNA nanotail, and the ECL of the CdS QDs was quenched by Fc, allowing the sensitive detection of miRNA-141. Once incubated with MMP-2, the Fc-peptide-ssDNA complexes were specifically cleaved, and the Fc molecules were released from the electrode surface, resulting in an obvious recovery of the CdS QD ECL signal. Through the "on-off-on" switching, MMP-2 was ultrasensitively detected as low as $33 \mathrm{fg} \mathrm{mL}^{-1}$. The biosensor showed excellent stability, reproducibility and selectivity, and performed well in real sample analysis.

As a label-free technique, field effect transistor (FET)-based biosensors have attracted great interest because of their extremely high sensitivity, fast response and miniaturization. The sensing events of FET-based biosensors mainly occur on a semiconducting channel, which is connected to two electrodes (source and drain electrode). The channel conductance between the source and drain electrode is controlled by a gate electrode. Charged molecules are immobilized on the surface of the semiconducting channel as probes; any changes caused by interaction with targets (such as the charge density or electrical double layer composition) may significantly influence the source-drain current, generating detectable signals. ${ }^{147}$ A variety of nanomaterials have been used for fabrication of FET-based biosensors, such as silicon nanowires (SiNWs), graphene and molybdenum disulfide $\left(\mathrm{MoS}_{2}\right)$. Choi et al. designed a SiNW-based FET biosensor for detecting MMP-2, in which SiNWs were prepared on an SOI wafer in a square-wave structure. ${ }^{143}$ The special structure of the SiNWs could provide a much more reactive surface than the linear structure, leading to improved sensitivity of the biosensor. 
MMP-2 specific peptide substrates with negatively charged peptide fragments $\left(\mathrm{Glu}_{6}\right)$ on the C-terminal (KKGGGGGGIPVSLRSG-EEEEEE) were immobilized on the aldehydefunctionalized SiNW surface for sensing. After being cleaved by MMP-2, peptide fragments containing $\mathrm{Glu}_{6}$ were removed from the SiNW surface, resulting in decreased conductance. The electric responses were dependent on the concentration of MMP-2 in the range of $1 \mathrm{pmol} \mathrm{L}^{-1}$ to $100 \mathrm{nmol} \mathrm{L}^{-1}$. Afterward, they coupled DNA modified AuNPs to MMP-2 specific peptide substrates; the negatively charged AuNPs and phosphate backbones of DNA drastically improved the sensitivity of the SiNW-FET biosensor. ${ }^{144}$ When the DNA-AuNP complexes were involved, the conductance change of SiNWs caused by peptide cleavage showed at least 10-fold enhancement, and MMP-2 could be detected in the range from $100 \mathrm{fmol} \mathrm{L}^{-1}$ to $10 \mathrm{nmol} \mathrm{L}^{-1}$. Similarly, Chen et al. constructed a rGO-based FET biosensor for sensitive detection of MMP-7 with a negatively charged polypeptide (JR2EC) as a substrate. ${ }^{145}$ The JR2EC peptide showed two cleavage sites for MMP-7, and exhibited reduced net charge after hydrolysis. Compared with $\mathrm{rGO}, \mathrm{MoS}_{2}$ is superior in monitoring the change of charged biomolecules, because of its significant band gap of $1.2 \mathrm{eV}$. Owing to the high affinity of $\mathrm{MoS}_{2}$ for physical and chemical adsorption of proteins, Park et al. developed a $\mathrm{MoS}_{2}$-based FET biosensor for ultrasensitive detection of MMP-9 by functionalizing the surface with amyloid- $\beta_{1-42}$ $\left(\mathrm{A} \beta_{1-42}\right.$ ) (as shown in Fig. 9D). ${ }^{146}$ In this biosensor, $A \beta_{1-42}$ served as not only a protein probe for MMP-9 proteolysis, but also an n-type dopant to amplify the electrical signals of the $\mathrm{MoS}_{2}$ FET. The abundant negative charge of $A \beta_{1-42}$ caused electrostatic n-doping in the $\mathrm{MoS}_{2}$ FET, resulting in enhanced electron carrier density. After being hydrolyzed by MMP-9, the length as well as the surface potential of $A \beta_{1-42}$ was decreased, thus the amplified electron carrier density of the $\mathrm{MoS}_{2}$ FET was reduced. Benefiting from the signal amplification effect, the $\mathrm{MoS}_{2}$ FET with $\mathrm{A} \beta_{1-42}$ achieved a large detection range for MMP-9 from $1 \mathrm{pmol} \mathrm{L}^{-1}$ to $10 \mathrm{nmol} \mathrm{L}{ }^{-1}$ with high precision.

3.2.3 Magnetic biosensors. Since biological samples have negligible magnetic background, magnetic biosensors serve as good candidates for sensitive detection of MMPs in clinical samples and in vivo. Compared with fluorescent and electrochemical biosensors, magnetic biosensors exhibit distinct advantages, such as negligible background noise, high stability and remote control ability. Superparamagnetic magnetic nanoparticles (MNPs) are the most widely used magnetic probes, which can efficiently magnify the magnetic resonance of protons in neighbouring water molecules. Aggregation of MNPs would accelerate the dephasing of water protons, causing the change of the proton relaxation time $\left(T_{2}\right)$. The dispersive MNPs display low relaxivity, while the aggregated MNPs possess high relaxivity; the change of the MNP state can be detected by monitoring $T_{2}$ with particular techniques. ${ }^{148}$ Therefore, magnetic relaxation switch (MRSw)-based biosensors have been developed for sensing MMPs. ${ }^{148-152}$ In Bhatia's work, biotin or neutravidin coated MNPs were modified with MMP peptide substrates containing PEG polymer tags to prevent their aggregation. After the peptides were cleaved by MMPs, the PEG polymers were detached from the MNP surface. Due to the specific binding between biotin and neutravidin, MNPs were clustered with decreased $T_{2} \cdot{ }^{149,150}$ Based on the MMP-induced aggregation of MNPs, a logical AND or OR sensing system could be created for MMP-2 and MMP-7. ${ }^{150}$ On the contrary, Gandhi et al. proposed a MMP-2 magnetic biosensor based on MMP-induced disassembly of iron oxide nanoparticles (IONPs). Neutravidin functionalized IONPs were cross-linked by biotin labeled MMP peptide substrates, resulting in the aggregation state. When incubated with MMPs, the clusters of IONPs were disintegrated into monodisperse nanoparticles, accompanied by an obviously changed magnetic response. The magnetic relaxation of IONPs was monitored by a magnetic particle spectrometer (MPS), and the field-dependent derivative of the magnetization $(\mathrm{d} m(H) / \mathrm{d} H)$ was measured. ${ }^{152}$ The biosensor could be used for cell secreted protease detection, and provided an alternative for distinguishing malignant phenotypes of solid tumors.

In addition to $T_{2}$ measurement, Zhu et al. proposed an activatable $T_{1}$ relaxivity biosensor by coupling $\mathrm{Gd}$ chelates to $\mathrm{Fe}_{3} \mathrm{O}_{4}$ NPs via MMP-2 specific peptide substrates. ${ }^{153}$ As shown in Fig. 10A, the $T_{1}$ relaxivity of Gd chelates was quenched by the local magnetic field produced by neighbor $\mathrm{Fe}_{3} \mathrm{O}_{4}$ NPs with $T_{2}$ relaxivity. After the linking peptides were cleaved by MMP-2, Gd chelates moved away from the local magnetic field of $\mathrm{Fe}_{3} \mathrm{O}_{4}$ NPs, resulting in recovery of the $T_{1}$ relaxivity. Moreover, a signal amplification strategy was introduced into the biosensor by coupling polyamidoamine (PAMAM) dendrimers on the peptide. The PAMAM dendrimers with multiple amino residues provided more binding sites for Gd chelates, and prolonged their rotational correlation time $\left(\tau_{\mathrm{R}}\right)$. Therefore, the sensitivity of the $T_{1}$ relaxivity recovery-based magnetic biosensor was effectively improved with a detection limit of $0.5 \mathrm{nmol} \mathrm{L}^{-1}$. MMP expression levels in tumor tissues were sensitively evaluated by the $\mathrm{Fe}_{3} \mathrm{O}_{4^{-}}$ pepA-Gd conjugates in situ via magnetic resonance imaging (MRI), providing valuable information for tumor diagnosis and metastasis monitoring. Differently, Schuerle et al. developed a magnetically actuated and thermosensitive MMP biosensor (MAPS), which realized remote activation at the disease site with the assistance of alternating magnetic fields (AMFs). ${ }^{154}$ As exhibited in Fig. 10B, in the biosensor, MNPs and MMP peptide substrates containing urinary reporter Cy7 were coencapsulated into thermosensitive liposomes. When exposed to AMFs, the MNPs in the liposomes underwent hysteretic heat dissipation, and the generated heat caused a local temperature increase and liposome deformation. Consequently, the MMP peptide substrates were released from the disrupted thermosensitive liposome, diffused to the target sites and were hydrolyzed by local MMPs. The uncleaved peptides were removed by exogenetic SA-beads with a magnet, thus the fluorescence of Cy7 indicated the activities of MMPs. The liposome could effectively prevent peptide substrates from nonspecific hydrolysis in the bloodstream, and facilitate their delivery to tumor sites via the EPR effect. Because of AMFs, the biosensor allowed deep-tissue activation without interference from biological processes. By analysing the urine of tumor-bearing mice, the MAPS biosensor was capable of distinguishing the MMP 
A

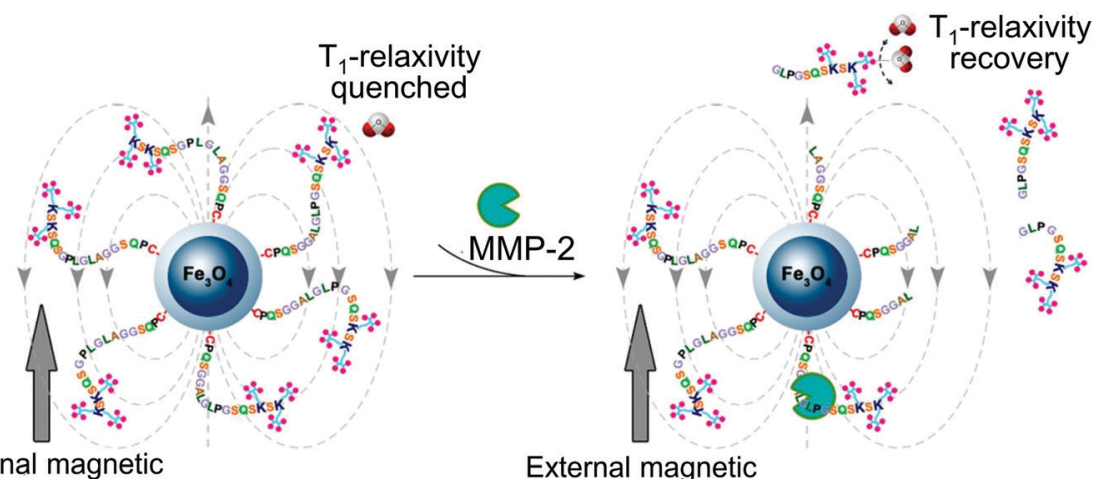

External magnetic field

MMP-2 cleavable peptide

Local magnetic Field by $\mathrm{Fe}_{3} \mathrm{O}_{4}$

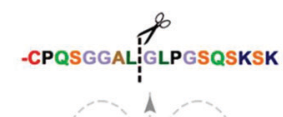

External magnetic

field

$\mathrm{H}_{2} \mathrm{O}$ molecule

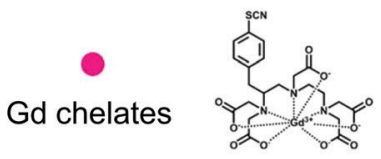

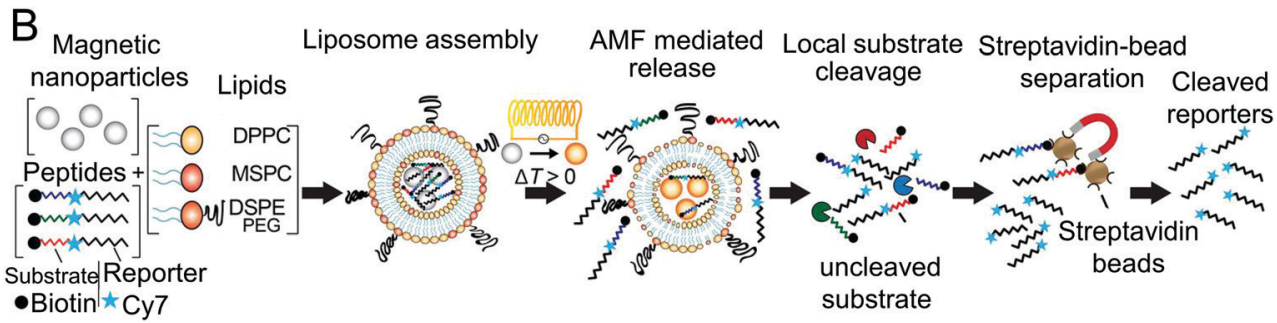

Fig. 10 (A) Activatable magnetic biosensor based on $\mathrm{Fe}_{3} \mathrm{O}_{4}$-peptide-Gd conjugates for determination of MMP-2 via monitoring the MMP cleavageinduced $T_{1}$ relaxivity recovery (reprinted with permission from ref. 153. Copyright 2017, American Chemical Society). (B) Design of the magnetically actuated and remotely addressable MMP biosensor for profiling MMP-2 activity in tumors (reprinted with permission from ref. 154. Copyright 2016, American Chemical Society).

expression profile in tumors, demonstrating the potential to classify clinical tumors for therapy selection. Sun et al. prepared an activatable MMP-2 probe by conjugating core/shell $\mathrm{Fe} / \mathrm{IONPs}$ with cleavable PEGylated peptide linkers. ${ }^{155}$ Based on the MMP cleavage-triggered colloidal behaviour changes in vivo, the probe performed well as an MRI contrast agent for sensitive and non-invasive monitoring of MMP-2 activity in vivo.

3.2.4 Photoacoustic biosensors. In vivo determination of MMP activities is of significant importance for deciphering their functions in diseases and developing specific therapy. The commonly used fluorescence biosensors are limited for in vivo application because of shallow tissue penetration. Photoacoustic (PA) imaging is a hybrid technique combining optical excitation with ultrasonic detection, allowing in vivo imaging of targets with sufficient penetration depth and high resolution. ${ }^{156}$ In recent years, several activatable PA biosensors have been developed for imaging MMPs on the basis of their proteolytic activities. ${ }^{157-163}$ Gambhir's group developed an activatable PA probe for visualizing MMPs in tumors, which was composed of a peptide (Ceeee[Ahx]PLGLAGrrrrrK) flanked by two chromophores (QXL680/Alexa750 and BHQ-3). ${ }^{157,158}$ The five arginine residues served as CPP for in vivo imaging. Two PA signals at $675 \mathrm{~nm}$ and $750 \mathrm{~nm}$ ascribed to BHQ-3 and Alexa750 were detected, allowing dual-wavelength imaging of MMPs. Prior to enzyme cleavage, subtraction of the images taken at the two wavelengths led to effective PA silence of the nonactivated probe. Cleavage of the probe by MMPs caused separation of the two chromophores, and the absorption and PA signals were changed. The peptide segments containing CPP and BHQ-3 were accumulated in cells; by subtraction of the images, a clear PA signal at $675 \mathrm{~nm}$ was observed. The PA biosensor performed well in noninvasive detection of follicular thyroid carcinoma.

Other than small-molecule dye-based PA probes, nanomaterials have also been employed to design PA biosensors for MMP determination, such as CuS nanoparticles (CuS NPs ${ }^{159}$ and gold-based nanostructures. ${ }^{160,161}$ As shown in Fig. 11A, Yang et al. constructed an activatable PA imaging probe by coupling BHQ-3 to CuS NPs via an MMP-cleavable peptide; the obtained CuS-peptide-BHQ-3 (CPQ) probe presented two distinct PA signals at $680 \mathrm{~nm}$ and $930 \mathrm{~nm} .{ }^{159}$ In the tumor sites, the peptide linker was cleaved by local MMPs, leading to the separation of BHQ-3 from the CuS NPs. As a small molecule, BHQ-3 was rapidly excreted, while the CuS NPs with large size remained for a longer time in the tumor. Therefore, the PA signal of BHQ-3 at $680 \mathrm{~nm}$ was decreased with time, while the PA signal of CuS NPs at $930 \mathrm{~nm}$ remained 

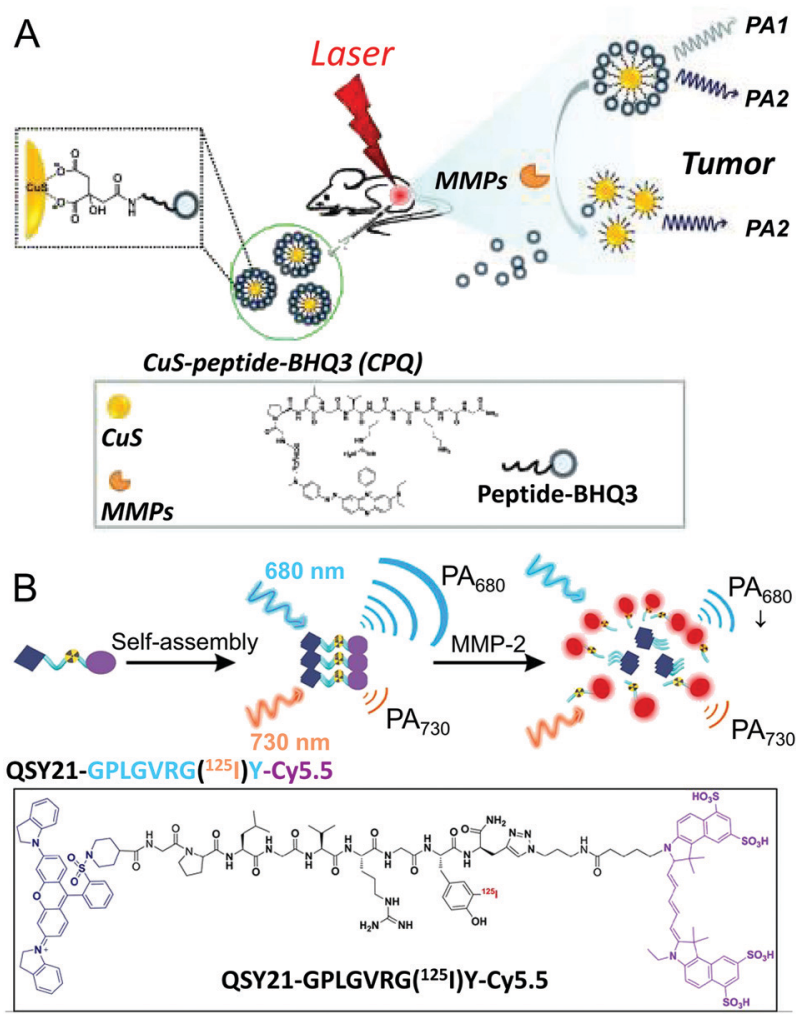

Fig. 11 (A) A CuS NP-based activatable PA imaging probe for visualization of MMP activity in vivo (reprinted with permission from ref. 159. Copyright 2014, Ivyspring International Publisher). (B) A ratiometric PA imaging probe with a QSY21-peptide-Cy5.5 conjugate for quantitatively visualizing MMP-2 in vivo (reprinted with permission from ref. 163. Copyright 2019, American Chemical Society).

nearly constant. The ratio of the PA signal at $680 \mathrm{~nm} / 930 \mathrm{~nm}$ was employed as an indicator for in vivo determination of the MMP activity. Compared with conventional fluorescence imaging, the CPQ probe for PA imaging exhibited dramatically improved tissue penetration and in vivo spatial resolution. Similarly, Liu et al. conjugated IRDye800 on gold nanorods (GNRs) via an MMP specific peptide. ${ }^{160}$ Xia et al. loaded MMP-2 polypeptide and IR-780 iodide on gold nanostars (GNS) through bovine serum albumin for NIF fluorescence and PA dual-modal imaging of MMP-2 activity. ${ }^{161}$

Besides the cleavage and retention strategy, self-assembly of small molecules paves a new way for constructing PA biosensors. For instance, Wang's group designed a gelatinase responsive small-molecule precursor (P18-PLGVRGRGD, P1), which consisted of NIR absorbing dye purpurin 18 (P18), a recognition sequence of gelatinase (PLGVRG) and a celltargeting ligand (RGD). ${ }^{162}$ After being cleaved by gelatinase, the residual P18 motifs self-assembled into nanofibers via intermolecular $\pi-\pi$ stacking, resulting in prolonged retention time in tumors and enhanced PA signals. Due to the assembly induced retention (AIR) effect and good tumor targeting ability, the PA probe showed better gelatinase response in imaging of tumors in vivo. Furthermore, this probe allowed photothermal therapy for tumor-bearing mice, and an improved therapeutic efficacy was obtained. Lately, Yin et al. constructed a ratiometric PA probe $(\mathrm{QC})$ for quantitatively detecting MMP-2 in vivo by linking Cy5.5 and QSY21 through an MMP-2 specific peptide substrate (as shown in Fig. 11B). ${ }^{163}$ Due to the amphiphilicity of QC molecules, they self-assembled into uniform nanoparticles in aqueous solution. Besides the quenched fluorescence of Cy5.5 resulting from FRET, the QC nanoparticles exhibited two PA signals at $680 \mathrm{~nm}$ and $730 \mathrm{~nm}$, attributed to Cy5.5 and QSY21, respectively. The aggregation state of QC was altered upon cleavage of the peptide linker, leading to changed PA signals. The PA signal at $680 \mathrm{~nm}$ was decreased with increasing MMP-2 concentration, while that at $730 \mathrm{~nm}$ remained almost unchanged, and served as an internal reference. A good linear relationship was observed between the ratio of $\mathrm{PAS}_{680} / \mathrm{PAS}_{730}$ and the MMP-2 concentration in the range of $10-640 \mathrm{ng} \mathrm{mL}^{-1}$. For in vivo imaging, the ratiometric PA signal responded to MMP-2 activity in a tumor sizedependent manner, allowing noninvasive evaluation of the MMP expression level for tumor diagnosis.

\subsection{Microfluidics-based platforms}

With the trend towards miniaturization of biosensors, microfluidics-based devices have attracted great interest to prepare POC biosensors. In microfluidics-based platforms, the whole experimental process including sample preparation, reaction, separation and detection is integrated together in microscale channels with a high degree of automation, significantly reducing the sample consumption and assay time. ${ }^{164}$ Additionally, by combining suitable detection techniques with microfluidic devices, the platforms are easily adjusted for high throughput analysis, allowing massively parallel detection with desired experimental conditions or simultaneous analysis of multianalytes. Considerable efforts have been devoted to developing microfluidics-based biosensors for determination of MMP activities, such as droplet microfluidics and microfluidic chips. ${ }^{165-178}$ Fluorescence detectors are prevalently used in microfluidic systems, owing to the advantages of high sensitivity and ease of implementation. In comparison with conventional reactions with microliter or milliliter-scale volume, droplet-based reactions with picoliter-scale reagent consumption exhibit high reaction efficiency due to the shorter diffusion distances. However, detecting low levels of protease activity in limited physiological samples is still challenging. To address this problem, Chen et al. introduced a biomolecule concentrator into a droplet-based microfluidics platform to analyse MMP-9 activity in mouse embryonic fibroblast (MEF) cell conditioned media. ${ }^{166}$ Specifically, MMPs were firstly concentrated in the main channel, then mixed with buffer and FRET-based peptide substrates, and encapsulated into droplets. The MMP-9 activity was determined by monitoring the fluorescence intensities of individual droplets, which increased with the MMP-9 concentration and assay time. With the preconcentration step, the concentration of MMP-9 in the droplets increased up to 16-fold, and the difference of the MMP-9 activity in stimulated and untreated cell samples was amplified about 10-fold, demonstrating the enhancement of the sensitivity. Moreover, the assay 
time was significantly reduced (ca. 10 times), and only $25 \mu \mathrm{L}$ diluted cell supernatant was required, which was 100-fold lower than conventional assays.

In addition to the fluorescence output by measuring the intensity, barcoding methods are superior in terms of multiplex and high-throughput analysis, which provide a specific graphic identifier for the corresponding target. Designed barcodes can be easily integrated into droplet-based microfluidic devices. As shown in Fig. 12A, Chen et al. employed one or more indicator dyes (Alexa-405 and Alexa-546) at specific concentrations to optically barcode the droplets, and created a ninemember barcoded droplet library (DL) for simultaneously monitoring protease activities in hundreds of droplets. ${ }^{168}$ The barcoded droplets containing a mixture of FRET-based MMP peptide substrates and specific inhibitor flowed into the picoinjector device and mixed $1: 1$ with the biological samples, generating new droplets comprising unique experimental conditions. Subsequently, hundreds of droplets flowed into the incubation chamber, and the fluorescence signals of the droplets were monitored by time-lapse fluorescence microscopy, and computationally processed by automated droplet tracking software. For determination of multiplex MMP activities, proteolytic activity matrix analysis (PrAMA) was introduced by deconvolution of the observed reaction rates. Consequently, MMP activities in clinical peritoneal fluid samples from patients with or without endometriosis were well determined and discriminated, suggesting promise for disease diagnosis. Although the droplet microfluidic involving barcoding technique shows multiplexing capability, it depends on the number of barcoding labels, limiting the multiplicity size of the assay. Chen et al. later introduced tandem sequential injection controls into the barcoding-based droplet microfluidic system; the developed pico-injector array increased the number of simultaneous enzyme reactions from 9 to 72 , dramatically improving the multiplexing capability. ${ }^{169}$ The optically barcoded DL was uploaded to an injector array with

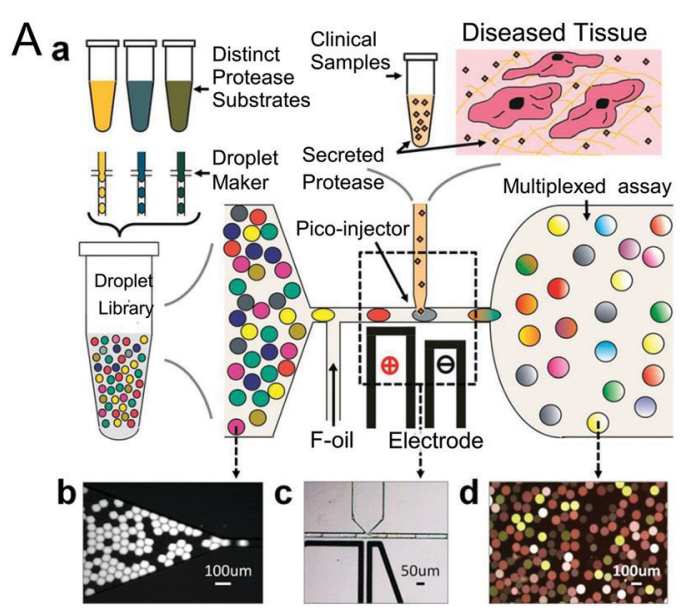

C
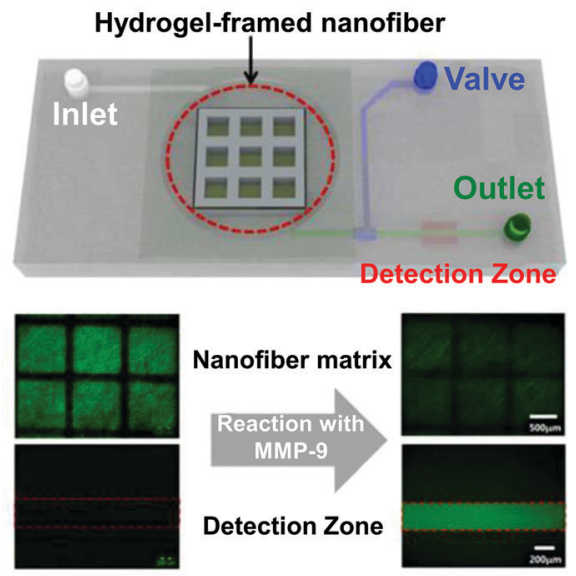

B

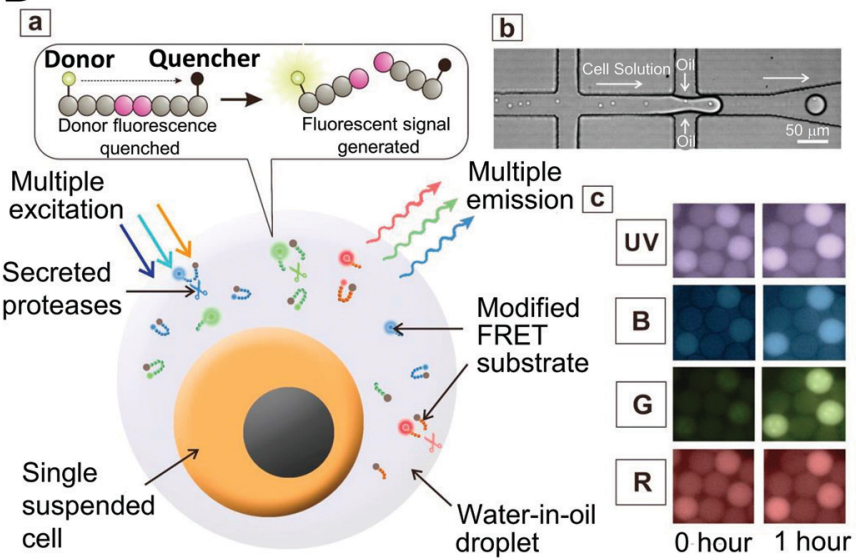

D (a)

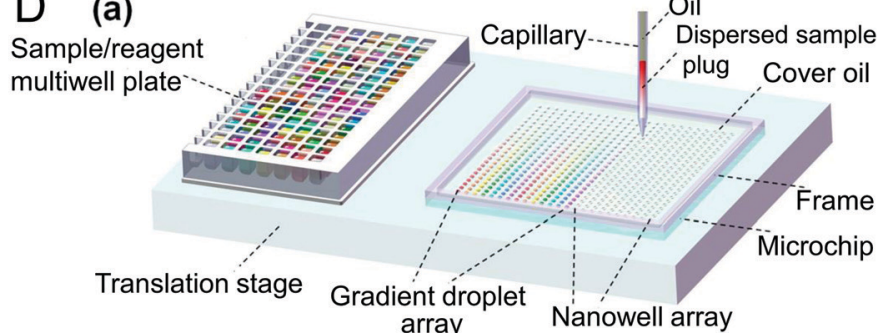

(b)

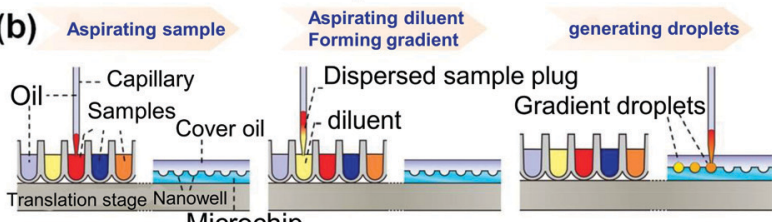

Fig. 12 (A) Schematic illustration of the droplet-based microfluidic platform integrated with the barcoding technique for determination of multiple MMP and ADAM activities in limited clinical samples (reprinted with permission from ref. 168. Copyright 2012, American Chemical Society). (B) Multi-color FRET peptide substrate based droplet microfluidic system for simultaneous determination of multiple MMP/ADAM activities from encapsulated single cells (reprinted with permission from ref. 172. Copyright 2016, Elsevier). (C) Hydrogel-framed microfluidic device for MMP-9 detection with a nanofiber matrix for peptide immobilization (reprinted with permission from ref. 176. Copyright 2016, American Chemical Society). (D) Schematic diagram of the microfluidic droplet gradient system and the procedures for generating the concentration gradient (reprinted with permission from ref. 178. Copyright 2019, American Chemical Society). 
3 pico-injectors, allowing combinatorial injections into individual droplets. Specific protease inhibitors were added by the pico-injectors to enhance the accuracy. Based on PrAMA inference, multiplex MMP/ADAM activities of MDA-MB-231 cells were well determined.

Effective detection of MMP activities in single cells is of vital importance for obtaining heterogeneity across individual cells and providing precision medicine requirements for disease. During the past five years, a series of microfluidics-based assays have been developed for analysing MMP activities at the single cell level. ${ }^{170-175}$ Jing et al. proposed an integrated microfluidics platform combining a droplet jetting generator with a deterministic lateral displacement (DLD) size-based sorting channel. ${ }^{170}$ Individual cells were encapsulated into the picoliter droplets with larger size and separated and proceeded into the observation chamber via the DLD channel, while the empty droplets with smaller size flowed directly to the waste outlet. Secreted MMP activities were evaluated by the FRET-based MMP peptide substrates in the droplets. For single leukocyte analysis, the DLD channel served as a washing machine to first remove the background activity in blood. ${ }^{171}$ For simultaneously detecting multiple protease activities in single cells, Chen's group encapsulated single cells into the droplets containing multi-color FRET-based peptide substrates (as exhibited in Fig. 12B). The hydrolysis reactions in the droplets were simultaneously monitored by fluorescence measurements at four distinct pairs of excitation and emission wavelengths, and specific MMP activities were inferred by PrAMA. ${ }^{172}$ By the integrated assay, the activities of six different proteases (MMP-2, MMP-3, MMP-9, ADAM-8, ADAM-10 and ADAM-17) in individual cells could be determined with high throughput ( $\sim 100$ cells per run) in both live-cell and in situ cell lysis manners. Furthermore, based on the measured six proteases' activity fingerprints of different cell lines in the brain tumor microenvironment, the assay was employed to perform real-time tumor analysis and screening. ${ }^{175}$ Owing to the high-throughput of the platform ( $\sim 100$ cells per second), a comprehensive characterization of tumor-associated cells could be completed within $2 \mathrm{~h}$, which was about 100-fold faster than the current gene sequence-based identification method.

In addition to droplet-based microfluidics, other types of microfluidics devices have also been reported for determining MMP activity, such as hydrogel-based microfluidics assays. ${ }^{176,177}$ Han et al. incorporated an electrospun nanofiber matrix into hydrogel micropatterns, and employed them as 3D nanostructured substrates for immobilizing FITC-labeled MMP-9 peptide substrates. ${ }^{176}$ As shown in Fig. 12C, the peptide loaded hydrogelframed nanofiber matrix was placed into the reaction chamber of a PDMS-based microfluidic system to monitor the reaction with MMP-9. After the immobilized fluorescent peptide substrates were cleaved by MMP-9, the resultant FITC-containing peptide fragments flowed to the detection zone for fluorescence measurement. Due to the large surface area of the nanofiber matrix for peptide loading and bidirectionally porous structures for easy access to MMP-9, the assay exhibited a faster response time (30 min) and lower detection limit $\left(10 \mathrm{pmol} \mathrm{L}^{-1}\right)$. Very recently, Wei et al. presented an interesting automated microfluidic dilution system for high throughput screening of MMP-9 inhibitors at the nanoliter level by integrating a droplet robot with a novel unilateral Taylor-Aris dispersion approach (as shown in Fig. 12D). ${ }^{178}$ The proposed system was able to automatically produce programmable concentration gradients in the range of ca. 6 orders of magnitude. By manipulating nanoliter droplets in a nanowell-array chip, the system realized miniaturized enzyme kinetic analysis and quantitative evaluation of the inhibition potencies of 102 compounds against MMP-9. The quantitative high throughput screening platform was superior in lower sample consumption and faster screening time, holding great promise for high throughput drug discovery.

\section{Other methods}

Besides the above described assays/biosensors, some other techniques have also been applied for quantifying MMPs, such as mass-spectrometry (MS)-based proteomic techniques, ${ }^{179-182}$ array/microarray-based assays, ${ }^{183-188}$ and nuclear imaging approaches. ${ }^{189-191}$

MS and liquid chromatography-tandem MS (LC-MS/MS) have evolved as promising techniques for qualitative and quantitative analysis of proteins via identification of the unique mass signature of the target. When determining active MMPs, unlabeled specific peptide substrates were cleaved by the MMPs, and then subjected to MS analysis. MMP activities are determined based on the $\mathrm{m} / \mathrm{z}$ ratio and corresponding peak intensities of ions produced by cleaved peptide debris. ${ }^{179,180}$ Kim et al. prepared a monolayer of AuNPs on a non-biofouling poly(oligo(ethylene glycol)methacrylate) (pOEGMA) film grafted $\mathrm{Si} / \mathrm{SiO}_{2}$ substrate, and then attached MMP-7 specific peptide substrates on the AuNP surface. ${ }^{179}$ After being treated with MMP-7, the cleaved peptide fragments residing on the AuNP surface were analyzed by time-of-flight secondary ion mass spectrometry (TOF-SIMS). The cleavage efficiency was linear with the logarithm of the MMP-7 concentration in the range of $20 \mathrm{ng} \mathrm{mL}^{-1}$ to $2 \mu \mathrm{g} \mathrm{mL}^{-1}$. Since the pOEGMA film could effectively prevent nonspecific protein adsorption, the sensitivity of the assay in serum was improved with a detection limit of $20 \mathrm{ng} \mathrm{mL}^{-1}$. As for determining the amount of total MMP protein, MMPs are directly digested by trypsin, and protein digestion was used for MS analysis. MMPs can be identified and quantified by analysing the mass signals of the intrinsic peptide. ${ }^{181,182}$ For instance, Ocaña et al. introduced an MB-based immunoaffinity enrichment process into the LC-MS/MS methods for determination of MMP-9 in 30 native mouse serum samples. ${ }^{181}$

Like microfluidics, microarrays are also superior in high throughput and automation. They are fabricated by spotting plenty of molecules on the substrate surface at high density, allowing conducting a large amount of parallel experiments simultaneously. Microarrays have achieved great success in enzyme profiling, such as evaluation of substrate specificity, determination of enzyme activity and screening of enzyme inhibitors. As for protease activity profiling, peptide microarrays 
are preferred. Kong et al. developed a phase transition-based peptide array for monitoring MMP-3 activity in real-time. ${ }^{183}$ TAMRA-labeled peptide substrates were immobilized on the array surface via cross-linking. After being treated with MMP-3, the cleaved fluorescent peptide segments were transferred from the solid phase to the liquid phase, and the peptide array was imaged by a portable fluorescence reader. The MMP-3 activity was associated with the cleaved fluorescent peptide concentration, which could be determined by analysing the fluorescence intensities of the array spots. The kinetic parameters of MMP-3, such as $K_{\mathrm{m}}, V_{\mathrm{max}}$, and $\mathrm{IC}_{50}$, were also determined by this method. Our group has also proposed a series of peptide microarray-based fluorescence arrays for multiplex determination of MMP activities and evaluation of MMP inhibitors. ${ }^{184-188}$ In our earlier work, a peptide microarray was fabricated by spotting biotin modified peptide substrates on a commercial chip slide with aldehyde groups. ${ }^{184}$ After being incubated with MMP-containing samples, FITC-labeled avidin was applied to the subarrays for fluorescence measurement. The fluorescence intensities of the spots decreased with increasing MMP activities, and the change of fluorescence intensity $(\Delta F)$ was proportional to the logarithm of the MMP-2 and MMP-9 concentration. The detection limit of the assay for MMP-2 and MMP-9 was $45 \mathrm{pg} \mathrm{mL}^{-1}$ and $60 \mathrm{pg} \mathrm{mL}{ }^{-1}$, respectively. Later, we prepared poly(glycidyl methacrylate-co-2hydroxyethyl methacrylate) (P(GMA-HEMA)) brush grafted glass slides, and employed them as substrates to fabricate a peptide microarray. ${ }^{185,186}$ Compared with two-dimensional planar epoxy slides, the P(GMA-HEMA) brush substrate showed higher loading capacity for biomolecules, and the 3D brush-like scaffold contributed to the improvement of the reaction efficiency. Additionally, the PHEMA on the brush substrate could effectively reduce nonspecific protein adsorption and cell adhesion, facilitating complex sample analysis. Thus, the fabricated peptide microarray on the P(GMA-HEMA) brush substrate exhibited higher sensitivity and lower detection limits for MMP-2 and MMP-9, and satisfactory results were obtained in cell sample analysis. To further improve the sensitivity, we prepared a novel Au/Ag@ $\mathrm{SiO}_{2}$ substrate with metal enhanced fluorescence (MEF) property for signal amplification, and the developed peptide microarray allowed multiplex determination of five MMPs' (MMP-2, -3, -7, -9, -14) activities. ${ }^{187}$ The Au/Ag@SiO ${ }_{2}$ substrate was covered by leaf-like $\mathrm{Ag}$ nanostructures, which not only increased the surface area for immobilization of more peptide probes, but also could enhance the fluorescence of dyes near the surface. Compared with glass slides, the $\mathrm{Au} / \mathrm{Ag} @ \mathrm{SiO}_{2}$ substrate showed 7 -fold and 5-fold fluorescence enhancement for Cy3 and FITC, respectively. Five specific FRET-based peptides with the FAM/Dabcyl pair were spotted on the Au/Ag@SiO substrate; after being cleaved by the target MMPs, the fluorescence of FAM was recovered and further enhanced by the substrate. Under the optimal conditions, the detection limits of the peptide microarray-based MEF assay were $12.2 \mathrm{fg} \mathrm{mL^{-1 }}$ for MMP-2, $102 \mathrm{fg} \mathrm{mL}{ }^{-1}$ for MMP-9, $60 \mathrm{pg} \mathrm{mL}{ }^{-1}$ for MMP-3, $0.22 \mathrm{pg} \mathrm{mL}^{-1}$ for MMP-7 and $0.68 \mathrm{ng} \mathrm{mL}^{-1}$ for MMP-14. Due to the high sensitivity and specificity, four extracellular MMPs' activities from seven different cell lines were determined by this assay. Using papillary thyroid carcinoma (PTC) as an example, five MMPs' activities of clinical thyroid tissues were profiled, and the relationship with the progression of PTC was evaluated. Very recently, we presented an array-based fluorescence assay for in situ multiplex profiling of five MMPs' activities in cell monolayers and tissue sections. ${ }^{188}$ Quenched FRET peptides containing CPP were directly spotted on cell monolayers or tissue sections; after being cleaved by extracellular MMPs, the peptide fragments with CPP and FAM moieties were taken up by cells, accompanied by recovered FAM fluorescence. Therefore, local MMP activities were determined directly from the fluorescence intensity of stained cells. Cell secreted MMPs' activities could be detected as low as hundreds of cells per square centimeter.

Nuclear imaging-based methods have also been developed for determination of MMP activities in vivo, such as positron emission tomography (PET) ${ }^{189}$ and single photon emission computed tomography (SPECT) ${ }^{190}$ which are based on molecular imaging of a radiolabelled tracer. For targeting active MMPs, the radiotracers were labeled on inhibitors ${ }^{189}$ or MMP peptide substrates. ${ }^{190,191}$ For instance, Schrigten et al. synthesized a series of radiofluorinated pyrimidine-2,4,6-triones with inhibition potency towards MMPs; one promising inhibitor was selected as MMP targeted radiotracers for noninvasive PET imaging of active MMPs in vivo. ${ }^{189}$ Watkins et al. designed an activatable SPECT imaging probe for detection of MMP-14 activity in vivo. ${ }^{190}$ The probe was constructed by conjugating a positively charged D-arginine octamer (r8) with a negatively charged attenuation sequence via an MMP-14 peptide substrate. R8 served as a CPP, and was radiolabeled by technetium-99m via the coordination interaction with a single amino acid chelate (SAAC). The attenuation sequence could minimize cellular uptake before cleavage, making the resultant probe circulate freely in vivo. After being recognized and cleaved by MMP-14, the peptide fragments with the CPP and radionuclide were transferred into cells, while the uncleaved probes and attenuation sequence-containing peptide segments were washed away. Thus, the MMP-14 activity was indicated by internalized radioactivity imaged with SPECT. Similarly, Duijnhoven et al. proposed a dual-isotope radiolabeled MMP-2/9 probe for imaging their activities in postischemic myocardial tissue. ${ }^{191}$ The probe was designed by inserting an MMP-2/9 peptide substrate between a ${ }^{177} \mathrm{Lu}$ labeled polycationic CPP and a ${ }^{125} \mathrm{I}$ labeled polyanionic inhibitory peptide, and further modified with an albumin binding ligand to prolong blood circulation. When the probe was activated by MMP-2/9, ${ }^{177} \mathrm{Lu}$ labeled CPPs were released and internalized into cells, while the polyanionic peptides with the corresponding radionuclide were cleared away. The local MMP-2/9 activities in tissue were determined by the ratio of ${ }^{177} \mathrm{Lu}$ to ${ }^{125} \mathrm{I}$; a high ratio suggested high uptake of the activated probe and high gelatinase activities. The probe was stable with the conjugation of ${ }^{125}$ I to polyanionic peptides, which effectively avoided potential intracellular-associated dehalogenation. Therefore, the dual-isotope radiolabeled probe performed well in determining the MMP-2/9 activity during remodeling post-myocardial infarction in vivo. 


\section{Conclusions and perspectives}

Accurate determination of MMPs both in vitro and in vivo would facilitate revealing the exact functions of MMPs in multiple biological events, promoting MMP-related studies. Herein, we provide an overview of current assays/biosensors for analysing MMPs, and highlight the latest progress in nanomaterial-based immunoassays and sensing techniques. As a golden standard for protein measurement, immunological methods integrated with multidisciplinary technology allow detection of MMPs with high sensitivity and specificity, but they cannot distinguish active MMPs from latent zymogens. Zymography is regarded as the longest-lived standard for determination of MMP activity, and can provide semi-quantitative information about different forms of MMPs. However, it is limited to a few kinds of MMPs, and the experimental procedure is quite complicated and time consuming. With the remarkable development in nanotechnology and transduction techniques, plentiful novel and facile biosensors based on proteolytic activity of MMPs have been proposed, such as optical biosensors, electrochemical biosensors, magnetic biosensors and PA biosensors. These biosensors exhibit high sensitivity and low detection limits, allowing analysis of MMPs in complex matrixes and in vivo. Furthermore, multiplex and high-throughput analysis of MMPs can be achieved by microfluidics and microarray-based assays.

Although significant progress has been made for measuring MMPs, some challenges still exist and improvements are needed to achieve better assay performance. Firstly, MMPs present overlapping substrate specificity due to their high homology, which affects the accuracy of the result. Design and screening of substrates with high specificity for particular MMPs is of vital importance for determining and distinguishing closely related MMPs in complex mixtures with high accuracy. Secondly, the sensitivity of biosensors should be improved to satisfy the demand for detecting trace MMPs in clinical samples. With the momentum of nanoscience, nanostructures with superior properties can not only serve as excellent signal transducers, but also as effective signal amplifiers, holding great promise for enhancing the sensitivity. Thirdly, nanomaterial-based probes have achieved certain success in monitoring MMP activities in vivo; the toxicities of nanoprobes should be well understood, especially the ones with longer retention time. Therefore, development of alternative nanoprobes with fast renal clearance or self-degradable capability is more likely to be demanded. Fourthly, for clinical diagnosis, more than one MMP should be involved to improve the diagnosis accuracy, thus simultaneous determination of multiple MMPs in a single sample is required. Microfluidic chips and microarrays with the advantages of automation and high throughput are good candidates for parallel analysis of multiple MMPs in a large number of samples. Finally, most of the methods are merely proof-of-concept verification in the laboratory under optimal experimental conditions; MMP biosensors for direct POC testing are still in their infancy. In the future, much more effort should be devoted to developing portable, simple and fast POC biosensors for determination of
MMPs in physiological samples (such as blood, serum and urine) with high sensitivity and specificity in a high throughput manner. New progress and major breakthroughs are expected to be made by the endeavour of researchers from different disciplines.

\section{Conflicts of interest}

There are no conflicts of interest to declare.

\section{Acknowledgements}

The authors would like to thank the National Natural Science Foundation of China (Grant no. 21775145) and Scientific Research Fundation of Wuhan Institute of Technology (K201940) for financial support.

\section{References}

1 H. Nagase and J. F. Woessner, J. Biol. Chem., 1999, 274, 21491-21494.

2 P. A. Snoek-vanBeurden and J. W. Von den Hoff, Biotechniques, 2005, 38, 73-83.

3 M. Egeblad and Z. Werb, Nat. Rev. Cancer, 2002, 2, 161-174.

4 S. Chakraborti, M. Mandal, S. Das, A. Mandal and T. Chakraborti, Mol. Cell. Biochem., 2003, 253, 269-285.

5 H. C. Crawford and L. M. Matrisian, Enzyme Protein, 1996, 49, 20-37.

6 C. J. Malemud, Front. Biosci., 2006, 11, 1696-1701.

7 Y. T. Konttinen, M. Ainola, H. Valleala, J. Ma, H. Ida, J. Mandelin, R. W. Kinne, S. Santavirta, T. Sorsa and C. López-Otín, Ann. Rheum. Dis., 1999, 58, 691-697.

8 L. C. Tetlow, D. J. Adlam and D. E. Woolley, Arthritis Rheum., 2001, 44, 585-594.

9 C. B. Jones, D. C. Sane and D. M. Herrington, Cardiovasc. Res., 2003, 59, 812-823.

10 J. B. Catterall and T. E. Cawston, Methods Mol. Biol., 2003, 225, 353-364.

11 C. Lombard, J. Saulnier and J. Wallach, Biochimie, 2005, 87, 265-272.

12 X. C. Cheng, H. Fang and W. F. Xu, J. Enzyme Inhib. Med. Chem., 2008, 23, 154-167.

13 K. Kupai, G. Szucs, S. Cseh, I. Hajdu, C. Csonka, T. Csont and P. Ferdinandy, J. Pharmacol. Toxicol. Methods, 2010, 61, 205-209.

14 S. Krizkova, O. Zitka, V. Adam, R. Kizek, M. Masarik, M. Stiborova, T. Eckschlager and G. J. Chavis, TrAC, Trends Anal. Chem., 2011, 30, 1819-1832.

15 A. Knapinska and G. B. Fields, ChemBioChem, 2012, 13, 2002-2020.

16 H. Lee and Y. P. Kim, BMB Rep., 2015, 48, 313-318.

17 A. Kirchhain, N. Poma, P. Salvo, L. Tedeschi, B. Melai, F. Vivaldi, A. Bonini, M. Franzini, L. Caponi, A. Tavanti and F. Di Francesco, TrAC, Trends Anal. Chem., 2019, 110, 35-50. 
18 S. Molet, C. Belleguic, H. Lena, N. Germain, C. Bertrand, S. Shapiro, J. M. Planquois, P. Delaval and V. Lagente, Inflammation Res., 2005, 54, 31-36.

19 M. Sulkala, T. Tervahartiala, T. Sorsa, M. Larmas, T. Salo and L. Tjäderhane, Arch. Oral Biol., 2007, 52, 121-127.

20 Q. Li, R. Zhang, Y. L. Ge, Y. W. Mei and Y. L. Guo, J. Mol. Neurosci., 2009, 38, 207-215.

21 A. Köhrmann, U. Kammerer, M. Kapp, J. Dietl and J. Anacker, BMC Cancer, 2009, 9, 188.

22 S. Mori, R. Pawankar, C. Ozu, M. Nonaka, T. Yagi and K. Okubo, Allergy, Asthma Immunol. Res., 2012, 4, 231-239.

23 A. Mazzoni, D. H. Pashley, F. R. Tay, P. Gobbi, G. Orsini, A. Ruggeri Jr, M. Carrilho, L. Tjäderhane, R. Di Lenarda and L. Breschi, J. Biomed. Mater. Res., Part A, 2009, 88, 697-703.

24 M. B. Ismail, E. de la Serna, G. Ruiz-Vega, T. García-Berrocoso, J. Montaner, M. Zourob, A. Othmane and E. Baldrich, Anal. Chim. Acta, 2018, 999, 144-154.

25 J. Liang, G. Lin, J. Tian, J. Chen, R. Liang, Z. Chen, Q. Deng, Z. Dong, T. Liu and Y. Wu, Clin. Chim. Acta, 2019, 490, 55-62.

26 X. Zhang, Z. Miao, Y. Hu, X. Yang, Y. Tang and D. Zhu, Sens. Actuators, B, 2018, 273, 511-518.

27 I. Ciani, H. Schulze, D. K. Corrigan, G. Henihan, G. Giraud, J. G. Terry, A. J. Walton, R. Pethig, P. Ghazal and J. Crain, Biosens. Bioelectron., 2012, 31, 413-418.

28 E. de la Serna, E. Martínez-García, T. García-Berrocoso, A. Penalba, A. Gil-Moreno, E. Colas, J. Montaner and E. Baldrich, Sens. Actuators, B, 2018, 269, 377-384.

29 G. Ruiz-Vega, A. García-Robaina, M. B. Ismail, H. Pasamar, T. García-Berrocoso, J. Montaner, M. Zourob, A. Othmane, F. J. del Campo and E. Baldrich, Biosens. Bioelectron., 2018, 115, 45-52.

30 G. Yang, L. Li, R. K. Rana and J. J. Zhu, Carbon, 2013, 61, 357-366.

31 B. S. Munge, J. Fisher, L. N. Millord, C. E. Krause, R. S. Dowd and J. F. Rusling, Analyst, 2010, 135, 1345-1350.

32 X. Ren, T. Zhang, D. Wu, T. Yan, X. Pang, B. Du, W. Lou and Q. Wei, Biosens. Bioelectron., 2017, 94, 694-700.

33 F. Jiang, J. J. Zhang, J. R. Zhang and J. J. Zhu, Analyst, 2013, 138, 1962-1965.

34 J. J. Shi, T. T. He, F. Jiang, E. Abdel-Halim and J. J. Zhu, Biosens. Bioelectron., 2014, 55, 51-56.

35 G. C. Fan, L. Han, H. Zhu, J. R. Zhang and J. J. Zhu, Anal. Chem., 2014, 86, 12398-12405.

36 X. Guo, J. Biophotonics, 2012, 5, 483-501.

37 O. R. Bolduc, J. N. Pelletier and J. F. O. Masson, Anal. Chem., 2010, 82, 3699-3706.

38 S. Mohseni, T. T. Moghadam, B. Dabirmanesh, S. Jabbari and K. Khajeh, Biosens. Bioelectron., 2016, 81, 510-516.

39 A. Tokarzewicz, L. Romanowicz, I. Sveklo and E. Gorodkiewicz, Anal. Methods, 2016, 8, 6428-6435.

40 A. Tokarzewicz, L. Romanowicz, I. Sveklo, E. Matuszczak, A. Hermanowicz and E. Gorodkiewicz, Anal. Methods, 2017, 9, 2407-2414.

41 Y. C. Huang, C. Y. Chiang, C. H. Li, T. C. Chang, C. S. Chiang, L. K. Chau, K. W. Huang, C. W. Wu, S. C. Wang and S. R. Lyu, Analyst, 2013, 138, 4599-4606.
42 W. T. Hsu, W. H. Hsieh, S. F. Cheng, C. P. Jen, C. C. Wu, C. H. Li, C. Y. Lee, W. Y. Li, L. K. Chau and C. Y. Chiang, Anal. Chim. Acta, 2011, 697, 75-82.

43 S. H. Wu, K. L. Lee, A. Chiou, X. Cheng and P. K. Wei, Small, 2013, 9, 3532-3540.

44 J. R. Lombardi and R. L. Birke, Acc. Chem. Res., 2009, 42, 734-742.

45 J. H. Granger, M. C. Granger, M. A. Firpo, S. J. Mulvihill and M. D. Porter, Analyst, 2013, 138, 410-416.

46 P. Zhao, H.-x. Li, D.-w. Li, Y.-j. Hou, L. Mao, M. Yang and Y. Wang, Talanta, 2019, 198, 527-533.

47 J. H. Verheijen, N. M. Nieuwenbroek, B. Beekman, R. Hanemaaijer, H. W. Verspaget, H. K. Ronday and A. H. Bakker, Biochem. J., 1997, 323, 603-609.

48 K. E. Hawkins, K. M. DeMars, C. Yang, G. A. Rosenberg and E. Candelario-Jalil, Mol. Brain, 2013, 6, 14.

49 S. Yan and E. Blomme, Vet. Pathol., 2003, 40, 227-236.

50 J. Banerjee, A. J. Hanson, E. K. Nyren-Erickson, B. Ganguli, A. Wagh, W. W. Muhonen, B. Law, J. B. Shabb, D. Srivastava and S. Mallik, Chem. Commun., 2010, 46, 3209-3211.

51 C. Q. Zhang, Y. M. Park, D. Yang, T. H. Yoo and H. C. Yoon, BioChip J., 2016, 10, 198-207.

52 E. Priyadarshini and N. Pradhan, Sens. Actuators, B, 2017, 238, 888-902.

53 Y. C. Chuang, J. C. Li, S. H. Chen, T. Y. Liu, C. H. Kuo, W. T. Huang and C. S. Lin, Biomaterials, 2010, 31, 6087-6095.

54 Y. C. Chuang, W. T. Huang, P. H. Chiang, M. C. Tang and C. S. Lin, Biosens. Bioelectron., 2012, 32, 24-31.

55 P. Chen, R. Selegård, D. Aili and B. Liedberg, Nanoscale, 2013, 5, 8973-8976.

56 G. B. Kim, K. H. Kim, Y. H. Park, S. Ko and Y. P. Kim, Biosens. Bioelectron., 2013, 41, 833-839.

57 C. Bremer, C. H. Tung and R. Weissleder, Nat. Med., 2001, 7, 743-748.

58 W. J. Akers, B. Xu, H. Lee, G. P. Sudlow, G. B. Fields, S. Achilefu and W. B. Edwards, Bioconjugate Chem., 2012, 23, 656-663.

59 M. Poreba, A. Szalek, W. Rut, P. Kasperkiewicz, I. RutkowskaWlodarczyk, S. J. Snipas, Y. Itoh, D. Turk, B. Turk and C. M. Overall, Sci. Rep., 2017, 7, 43135.

60 X. Hai, X. Wang, M. El-Attug, E. Adams, J. Hoogmartens and A. Van Schepdael, Anal. Chem., 2010, 83, 425-430.

61 A. Siennicka, M. Zuchowski, K. Chełstowski, M. Cnotliwy, J. S. Clark and M. Jastrzębska, BioMed Res. Int., 2018, 3205324.

62 K. B. Fonseca, F. R. Maia, F. A. Cruz, D. Andrade, M. A. Juliano, P. L. Granja and C. C. Barrias, Soft Matter, 2013, 9, 3283-3292.

63 S. Y. Li, L. H. Liu, H. Cheng, B. Li, W. X. Qiu and X. Z. Zhang, Chem. Commun., 2015, 51, 14520-14523.

64 K. J. Son, D. S. Shin, T. Kwa, Y. Gao and A. Revzin, Anal. Chem., 2013, 85, 11893-11901.

65 J. L. Leight, D. L. Alge, A. J. Maier and K. S. Anseth, Biomaterials, 2013, 34, 7344-7352.

66 H. Cheng, S. Y. Li, H. R. Zheng, C. X. Li, B. R. Xie, K. W. Chen, B. Li and X. Z. Zhang, Anal. Chem., 2017, 89, 4349-4354. 
67 S. Lee, J. H. Ryu, K. Park, A. Lee, S. Y. Lee, I. C. Youn, C. H. Ahn, S. M. Yoon, S. J. Myung, D. H. Moon, X. Chen, K. Choi, I. C. Kwon and K. Kim, Nano Lett., 2009, 9, 4412-4416.

68 L. Zhu, F. Zhang, Y. Ma, G. Liu, K. Kim, X. Fang, S. Lee and X. Chen, Mol. Pharmaceutics, 2011, 8, 2331-2338.

69 J. Park, J. Yang, E. K. Lim, E. Kim, J. Choi, J. K. Ryu, N. H. Kim, J. S. Suh, J. I. Yook and Y. M. Huh, Angew. Chem., Int. Ed., 2012, 51, 945-948.

70 A. Lee, S. J. Choi, K. Park, J. W. Park, K. Kim, K. Choi, S. Y. Yoon and I. Youn, Bioconjugate Chem., 2013, 24, 1068-1074.

71 L. Zhu, Y. Ma, D. O. Kiesewetter, Y. Wang, L. Lang, S. Lee, G. Niu and X. Chen, ACS Chem. Biol., 2014, 9, 510-516.

72 R. Lee, S. J. Choi, K. C. Moon, J. W. Park, K. Kim, S. Y. Yoon and I. Youn, ACS Biomater. Sci. Eng., 2019, 5, 3039-3048.

73 A. Megia-Fernandez, B. Mills, C. Michels, S. V. Chankeshwara, N. Krstajić, C. Haslett, K. Dhaliwal and M. Bradley, Org. Biomol. Chem., 2018, 16, 8056-8063.

74 L. Yin, H. Sun, M. Zhao, A. Wang, S. Qiu, Y. Gao, J. Ding, S. J. Ji, H. Shi and M. Gao, J. Org. Chem., 2019, 84, 6126-6133.

75 S. Lee, E. J. Cha, K. Park, S. Y. Lee, J. K. Hong, I. C. Sun, S. Y. Kim, K. Choi, I. C. Kwon, K. Kim and C. H. Ahn, Angew. Chem., Int. Ed., 2008, 47, 2804-2807.

76 Y. P. Kim, Y. H. Oh, E. Oh, S. Ko, M. K. Han and H. S. Kim, Anal. Chem., 2008, 80, 4634-4641.

77 X. Xia, M. Yang, L. K. Oetjen, Y. Zhang, Q. Li, J. Chen and Y. Xia, Nanoscale, 2011, 3, 950-953.

78 X. Wang, Y. Xia, Y. Liu, W. Qi, Q. Sun, Q. Zhao and B. Tang, Chem. - Eur. J., 2012, 18, 7189-7195.

79 Y. Hong, M. Ku, D. Heo, S. Hwang, E. Lee, J. Park, J. Choi, H. J. Lee, M. Seo and E. J. Lee, Biosens. Bioelectron., 2014, 57, 171-178.

80 M. Ku, Y. Hong, D. Heo, E. Lee, S. Hwang, J.-S. Suh and J. Yang, Biosens. Bioelectron., 2016, 77, 471-477.

81 M. Luan, N. Li, W. Pan, L. Yang, Z. Yu and B. Tang, Chem. Commun., 2017, 53, 356-359.

82 X. Gao, L. Jiang, B. Hu, F. Kong, X. Liu, K. Xu and B. Tang, Anal. Chem., 2018, 90, 4719-4724.

83 E. Song, D. Cheng, Y. Song, M. Jiang, J. Yu and Y. Wang, Biosens. Bioelectron., 2013, 47, 445-450.

84 Z. Yue, P. Lv, H. Yue, Y. Gao, D. Ma, W. Wei and G. Ma, Chem. Commun., 2013, 49, 3902-3904.

85 D. Feng, Y. Zhang, T. Feng, W. Shi, X. Li and H. Ma, Chem. Commun., 2011, 47, 10680-10682.

86 G. Xi, X. Wang and T. Chen, Int. J. Nanomed., 2016, 11, 1537-1547.

87 J. Li, C. H. Lu, Q. H. Yao, X. L. Zhang, J. J. Liu, H. H. Yang and G. N. Chen, Biosens. Bioelectron., 2011, 26, 3894-3899.

88 P. D. Nguyen, V. T. Cong, C. Baek and J. Min, Biosens. Bioelectron., 2017, 89, 666-672.

89 Y. Huang, M. Shi, K. Hu, S. Zhao, X. Lu, Z. F. Chen, J. Chen and H. Liang, J. Mater. Chem. B, 2013, 1, 3470-3476.

90 J. Xu, L. Fang, M. Shi, Y. Huang, L. Yao, S. Zhao, L. Zhang and H. Liang, Chem. Commun., 2019, 55, 1651-1654.
91 T. Ma, Y. Hou, J. Zeng, C. Liu, P. Zhang, L. Jing, D. Shangguan and M. Gao, J. Am. Chem. Soc., 2017, 140, 211-218.

92 E. Y. Chung, C. J. Ochs, Y. Wang, L. Lei, Q. Qin, A. M. Smith, A. Y. Strongin, R. Kamm, Y. X. Qi and S. Lu, Nano Lett., 2015, 15, 5025-5032.

93 Y. Wang, T. Lin, W. Zhang, Y. Jiang, H. Jin, H. He, V. C. Yang, Y. Chen and Y. Huang, Theranostics, 2015, 5, 787-795.

94 X. Li, D. Deng, J. Xue, L. Qu, S. Achilefu and Y. Gu, Biosens. Bioelectron., 2014, 61, 512-518.

95 Y. Wang, P. Shen, C. Li, Y. Wang and Z. Liu, Anal. Chem., 2012, 84, 1466-1473.

96 M. He and Z. Liu, Anal. Chem., 2013, 85, 11691-11694.

97 Y. C. Chan, C. W. Chen, M. H. Chan, Y. C. Chang, W. M. Chang, L. H. Chi, H. M. Yu, Y. F. Lin, D. P. Tsai and R. S. Liu, Biosens. Bioelectron., 2016, 80, 131-139.

98 S. Cao, Z. Li, J. Zhao, M. Chen and N. Ma, ACS Sens., 2018, 3, 1522-1530.

99 W. Yang, G. Zhang, W. Weng, B. Qiu, L. Guo, Z. Lin and G. Chen, RSC Adv., 2014, 4, 58852-58857.

100 S. Y. Kwak, J. K. Yang, S. J. Jeon, H. I. Kim, J. Yim, H. Kang, S. Kyeong, Y. S. Lee and J. H. Kim, Adv. Funct. Mater., 2014, 24, 5119-5128.

101 J. K. Yang, S. Y. Kwak, S. J. Jeon, E. Lee, J. M. Ju, H. I. Kim, Y. S. Lee and J. H. Kim, Nanoscale, 2016, 8, 12272-12281.

102 J. K. Yang, I. J. Hwang, S. J. Jeon, J. M. Ju, H. I. Kim, D. Yim, Y. S. Lee and J. H. Kim, Sens. Actuators, B, 2019, 284, 485-493.

103 Y. Zhang, G. Wang, L. Yang, F. Wang and A. Liu, Coord. Chem. Rev., 2018, 370, 1-21.

104 K. P. Loh, Q. Bao, G. Eda and M. Chhowalla, Nat. Chem., 2010, 2, 1015-1024.

105 G. B. Kim and Y. P. Kim, Theranostics, 2012, 2, 127-138.

106 Z. Wang, X. Li, D. Feng, L. Li, W. Shi and H. Ma, Anal. Chem., 2014, 86, 7719-7725.

107 X. Yu, K. Wen, Z. Wang, X. Zhang, C. Li, S. Zhang and J. Shen, Anal. Chem., 2016, 88, 3512-3520.

108 Y. P. Kim, W. L. Daniel, Z. Xia, H. Xie, C. A. Mirkin and J. Rao, Chem. Commun., 2010, 46, 76-78.

109 H. Yao, Y. Zhang, F. Xiao, Z. Xia and J. Rao, Angew. Chem., Int. Ed., 2007, 46, 4346-4349.

110 Z. Xia, Y. Xing, M. K. So, A. L. Koh, R. Sinclair and J. Rao, Anal. Chem., 2008, 80, 8649-8655.

111 Z. Xia, Y. Xing, J. Jeon, Y. P. Kim, J. Gall, A. DragulescuAndrasi, S. S. Gambhir and J. Rao, ACS Chem. Biol., 2011, 6, 1117-1126.

112 H. Su, M. Zang, L. Lu and F. Li, Chem. Commun., 2019, 55, 7085-7088.

113 S. H. Jung, D. H. Kong, J. H. Park, S. T. Lee, J. Hyun, Y. M. Kim and K. S. Ha, Analyst, 2010, 135, 1050-1057.

114 Y. Hong, M. Ku, E. Lee, J. S. Suh, Y. M. Huh, D. S. Yoon and J. Yang, J. Biomed. Opt., 2013, 19, 1-7.

115 J. Park, G. Baik Kim, A. Lippitz, Y. M. Kim, D. Jung, W. E. S. Unger, Y. P. Kim and T. G. Lee, Sens. Actuators, $B, 2019,281,527-534$.

116 T. Gong, K. V. Kong, D. Goh, M. Olivo and K. T. Yong, Biomed. Opt. Express, 2015, 6, 2076-2087. 
117 T. Gong, Z. Y. Hong, C. H. Chen, C. Y. Tsai, L. D. Liao and K. V. Kong, ACS Nano, 2017, 11, 3365-3375.

118 D. Lin, C. Y. Tseng, Q. F. Lim, M. J. Tan and K. V. Kong, J. Mater. Chem. B, 2018, 6, 2536-2540.

119 S. Arshavsky-Graham, N. Massad-Ivanir, E. Segal and S. Weiss, Anal. Chem., 2019, 91, 441-467.

120 L. Gao, N. Mbonu, L. Cao and D. Gao, Anal. Chem., 2008, 80, 1468-1473.

121 K. A. Kilian, L. M. H. Lai, A. Magenau, S. Cartland, T. Böcking, N. Di Girolamo, M. Gal, K. Gaus and J. J. Gooding, Nano Lett., 2009, 9, 2021-2025.

122 A. H. Soeriyadi, B. Gupta, P. J. Reece and J. J. Gooding, Polym. Chem., 2014, 5, 2333-2341.

123 B. Gupta, K. Mai, S. B. Lowe, D. Wakefield, N. Di Girolamo, K. Gaus, P. J. Reece and J. J. Gooding, Anal. Chem., 2015, 87, 9946-9953.

124 F. S. Krismastuti, S. Pace and N. H. Voelcker, Adv. Funct. Mater., 2014, 24, 3639-3650.

125 F. S. H. Krismastuti, A. Cavallaro, B. Prieto-Simon and N. H. Voelcker, Adv. Sci., 2016, 3, 1500383.

126 F. S. H. Krismastuti, M. R. Dewi, B. Prieto-Simon, T. Nann and N. H. Voelcker, ACS Sens., 2017, 2, 203-209.

127 J. Lee, J. Y. Yun, W. C. Lee, S. Choi, J. Lim, H. Jeong, D. S. Shin and Y. J. Park, Sens. Actuators, B, 2017, 240, 735-741.

128 G. Liu, J. Wang, D. S. Wunschel and Y. Lin, J. Am. Chem. Soc., 2006, 128, 12382-12383.

129 D. S. Shin, Y. Liu, Y. Gao, T. Kwa, Z. Matharu and A. Revzin, Anal. Chem., 2013, 85, 220-227.

130 Y. Zheng and Z. Ma, Biosens. Bioelectron., 2018, 108, 46-52.

131 D. Wang, Y. Chai, Y. Yuan and R. Yuan, Anal. Chem., 2017, 89, 8951-8956.

132 P. Jing, H. Yi, S. Xue, R. Yuan and W. Xu, $R S C A d v ., 2015,5$, 65725-65730.

133 D. Wang, Y. Yuan, Y. Zheng, Y. Chai and R. Yuan, Chem. Commun., 2016, 52, 5943-5945.

134 B. B. Kou, L. Zhang, H. Xie, D. Wang, Y. L. Yuan, Y. Q. Chai and R. Yuan, ACS Appl. Mater. Interfaces, 2016, 8, 22869-22874.

135 W. Xu, P. Jing, H. Yi, S. Xue and R. Yuan, Sens. Actuators, B, 2016, 230, 345-352.

136 B. B. Kou, Y. Q. Chai, Y. L. Yuan and R. Yuan, Anal. Chem., 2017, 89, 9383-9387.

137 T. Zheng, R. Zhang, Q. Zhang, T. Tan, K. Zhang, J. J. Zhu and H. Wang, Chem. Commun., 2013, 49, 7881-7883.

138 T. B. Tran, P. D. Nguyen, C. Baek and J. Min, Biosens. Bioelectron., 2016, 77, 631-637.

139 A. Biela, M. Watkinson, U. C. Meier, D. Baker, G. Giovannoni, C. R. Becer and S. Krause, Biosens. Bioelectron., 2015, 68, 660-667.

140 Q. Dang, H. Gao, Z. Li, H. Qi, Q. Gao and C. Zhang, Anal. Bioanal. Chem., 2016, 408, 7067-7075.

141 H. Gao, Q. Dang, S. Xia, Y. Zhao, H. Qi, Q. Gao and C. Zhang, Sens. Actuators, B, 2017, 253, 69-76.

142 Y. Nie, P. Zhang, H. Wang, Y. Zhuo, Y. Chai and R. Yuan, Anal. Chem., 2017, 89, 12821-12827.
143 J. H. Choi, H. Kim, H. S. Kim, S. H. Um, J. W. Choi and B. K. Oh, J. Biomed. Nanotechnol., 2013, 9, 732-735.

144 J. H. Choi, H. Kim, J. H. Choi, J. W. Choi and B. K. Oh, ACS Appl. Mater. Interfaces, 2013, 5, 12023-12028.

145 H. Chen, P. Chen, J. Huang, R. Selegård, M. Platt, A. Palaniappan, D. Aili, A. I. Y. Tok and B. Liedberg, Anal. Chem., 2016, 88, 2994-2998.

146 H. Park, H. Lee, S. H. Jeong, E. Lee, W. Lee, N. Liu, D. S. Yoon, S. Kim and S. W. Lee, Anal. Chem., 2019, 91, 8252-8258.

147 R. Ahmad, T. Mahmoudi, M. S. Ahn and Y. B. Hahn, Biosens. Bioelectron., 2018, 100, 312-325.

148 A. Sobczak-Kupiec, J. Venkatesan, A. A. AlAnezi, D. Walczyk, A. Farooqi, D. Malina, S. H. Hosseini and B. Tyliszczak, Nanomedicine, 2016, 12, 2459-2473.

149 T. J. Harris, G. von Maltzahn, A. M. Derfus, E. Ruoslahti and S. N. Bhatia, Angew. Chem., Int. Ed., 2006, 45, 3161-3165.

150 G. von Maltzahn, T. J. Harris, J. H. Park, D. H. Min, A. J. Schmidt, M. J. Sailor and S. N. Bhatia, J. Am. Chem. Soc., 2007, 129, 6064-6065.

151 S. Matsumura, I. Aoki, T. Saga and K. Shiba, Mol. Pharmaceutics, 2011, 8, 1970-1974.

152 S. Gandhi, H. Arami and K. M. Krishnan, Nano Lett., 2016, 16, 3668-3674.

153 X. Zhu, H. Lin, L. Wang, X. Tang, L. Ma, Z. Chen and J. Gao, ACS Appl. Mater. Interfaces, 2017, 9, 21688-21696.

154 S. Schuerle, J. S. Dudani, M. G. Christiansen, P. Anikeeva and S. N. Bhatia, Nano Lett., 2016, 16, 6303-6310.

155 Z. Sun, K. Cheng, Y. Yao, F. Wu, J. Fung, H. Chen, X. Ma, Y. Tu, L. Xing and L. Xia, ACS Nano, 2019, 13, 1153-1167.

156 Q. Miao and K. Pu, Bioconjugate Chem., 2016, 27, 2808-2823.

157 J. Levi, S. R. Kothapalli, T. J. Ma, K. Hartman, B. T. KhuriYakub and S. S. Gambhir, J. Am. Chem. Soc., 2010, 132, 11264-11269.

158 J. Levi, S. R. Kothapalli, S. Bohndiek, J. K. Yoon, A. Dragulescu-Andrasi, C. Nielsen, A. Tisma, S. Bodapati, G. Gowrishankar and X. Yan, Clin. Cancer Res., 2013, 19, 1494-1502.

159 K. Yang, L. Zhu, L. Nie, X. Sun, L. Cheng, C. Wu, G. Niu, X. Chen and Z. Liu, Theranostics, 2014, 4, 134-141.

160 C. Liu, Y. Yang, Z. Qiu, Y. Huang and L. Sun, 2015 IEEE International Ultrasonics Symposium (IUS), 2015, vol. 1.

161 F. Xia, J. Niu, Y. Hong, C. Li, W. Cao, L. Wang, W. Hou, Y. Liu and D. Cui, Acta Biomater., 2019, 89, 289-299.

162 D. Zhang, G. B. Qi, Y. X. Zhao, S. L. Qiao, C. Yang and H. Wang, Adv. Mater., 2015, 27, 6125-6130.

163 L. Yin, H. Sun, H. Zhang, L. He, L. Qiu, J. Lin, H. Xia, Y. Zhang, S. Ji and H. Shi, J. Am. Chem. Soc., 2019, 141, 3265-3273.

164 X. Weng, Y. Kang, Q. Guo, B. Peng and H. Jiang, Biosens. Bioelectron., 2019, 132, 171-185.

165 S. Jambovane, D. J. Kim, E. C. Duin, S. K. Kim and J. W. Hong, Anal. Chem., 2011, 83, 3358-3364.

166 C. H. Chen, A. Sarkar, Y. A. Song, M. A. Miller, S. J. Kim, L. G. Griffith, D. A. Lauffenburger and J. Han, J. Am. Chem. Soc., 2011, 133, 10368-10371.

167 T. D. Rane, H. C. Zec and T. H. Wang, Anal. Chem., 2015, 87, 1950-1956. 
168 C. H. Chen, M. A. Miller, A. Sarkar, M. T. Beste, K. B. Isaacson, D. A. Lauffenburger, L. G. Griffith and J. Han, J. Am. Chem. Soc., 2012, 135, 1645-1648.

169 E. X. Ng, M. A. Miller, T. Jing, D. A. Lauffenburger and C. H. Chen, Lab Chip, 2015, 15, 1153-1159.

170 T. Jing, R. Ramji, M. E. Warkiani, J. Han, C. T. Lim and C. H. Chen, Biosens. Bioelectron., 2015, 66, 19-23.

171 T. Jing, Z. Lai, L. Wu, J. Han, C. T. Lim and C. H. Chen, Anal. Chem., 2016, 88, 11750-11757.

172 E. X. Ng, M. A. Miller, T. Jing and C. H. Chen, Biosens. Bioelectron., 2016, 81, 408-414.

173 Y. C. Chen, Y. H. Cheng, P. Ingram and E. Yoon, Sci. Rep., 2016, 6, 27154.

174 Z. Yu, L. Zhou, T. Zhang, R. Shen, C. Li, X. Fang, G. Griffiths and J. Liu, ACS Sens., 2017, 2, 626-634.

175 E. X. Ng, G. Sun, S. C. Wei, M. A. Miller, R. DasGupta, P. Y. P. Lam and C. H. Chen, Anal. Chem., 2018, 91, 1277-1285.

176 S. W. Han and W. G. Koh, Anal. Chem., 2016, 88, 6247-6253.

177 D. Puchberger-Enengl, C. Krutzler, F. Keplinger and M. J. Vellekoop, Lab Chip, 2014, 14, 378-383.

178 Y. Wei, Y. Zhu and Q. Fang, Anal. Chem., 2019, 91, 4995-5003.

179 Y. P. Kim, B. S. Lee, E. Kim, I. S. Choi, D. W. Moon, T. G. Lee and H. S. Kim, Anal. Chem., 2008, 80, 5094-5102.

180 S. Bregant, C. Huillet, L. Devel, A. S. Dabert-Gay, F. Beau, R. Thai, B. Czarny, A. Yiotakis and V. Dive, J. Proteome Res., 2009, 8, 2484-2494.
181 M. F. Ocaña and H. Neubert, Anal. Biochem., 2010, 399, 202-210.

182 L. M. Prely, K. Paal, J. Hermans, S. van der Heide, A. J. van Oosterhout and R. Bischoff, J. Chromatogr. A, 2012, 1246, 103-110.

183 D. H. Kong, S. H. Jung, S. T. Lee, Y. M. Kim and K. S. Ha, Biosens. Bioelectron., 2012, 36, 147-153.

184 Z. Lei, J. Gao, X. Liu, D. Liu and Z. Wang, Anal. Methods, 2016, 8, 72-77.

185 Z. Lei, J. Gao, X. Liu, D. Liu and Z. Wang, ACS Appl. Mater. Interfaces, 2016, 8, 10174-10182.

186 Z. Lei, H. Chen, H. Zhang, Y. Wang, X. Meng and Z. Wang, ACS Appl. Mater. Interfaces, 2017, 9, 44241-44250.

187 Z. Lei, H. Zhang, Y. Wang, X. Meng and Z. Wang, Anal. Chem., 2017, 89, 6749-6757.

188 Z. Lei, M. Jian, J. Wei, Y. Wang, X. Meng and Z. Wang, Anal. Chim. Acta, 2019, 1078, 112-118.

189 D. Schrigten, H. J. r. Breyholz, S. Wagner, S. Hermann, O. Schober, M. Schäfers, G. n. Haufe and K. Kopka, J. Med. Chem., 2011, 55, 223-232.

190 G. A. Watkins, E. F. Jones, M. S. Shell, H. F. VanBrocklin, M. H. Pan, S. M. Hanrahan, J. J. Feng, J. He, N. E. Sounni and K. A. Dill, Bioorg. Med. Chem., 2009, 17, 653-659.

191 S. M. van Duijnhoven, M. S. Robillard, S. Hermann, M. T. Kuhlmann, M. Schäfers, K. Nicolay and H. Grüll, Mol. Pharmaceutics, 2014, 11, 1415-1423. 